\title{
Stochastic Inverse Methods to Identify Non-Gaussian Model Parameters in Heterogeneous Aquifers
}

\author{
PhD Thesis submitted by \\ Haiyan Zhou \\ Advisor \\ J. Jaime Gómez-Hernández
}

September 2011

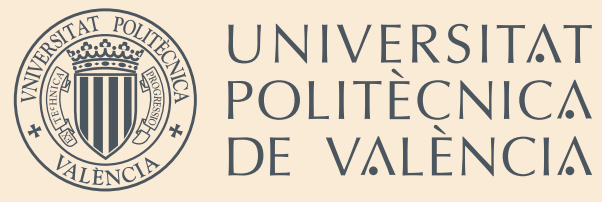





\title{
Stochastic Inverse Methods to Identify Non-Gaussian Model Parameters in Heterogeneous Aquifers
}

\author{
PhD Thesis submitted by \\ Haiyan Zhou \\ Advisor \\ J. Jaime Gómez-Hernández \\ PhD Program
}

Ingeniería del Agua y Medioambiental

Grupo de Hidrogeologia

Departamento de Ingeniería Hidráulica y Medio

Ambiente

Universitat Politècnica de València

September 2011, Valencia, Spain
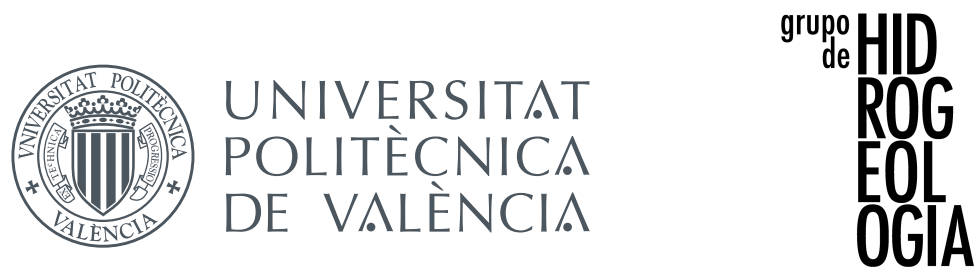
(C) Copyright by Haiyan Zhou 2011 All rights reserved. 
To Liangping 


\section{Abstract}

Numerical modeling of groundwater flow and mass transport is increasingly becoming a reference criterion nowadays for water resources assessment and environmental protection. To render the model reliable for future predictions, the model structure and parameters have to be characterized as close to the reality as possible. The process of model identification by integrating measured parameters and observed model states is so called inverse problem. A series of methods has been proposed to solve the inverse problem in the past several decades and its evolution is discussed in the thesis. The main point of this thesis is to propose two stochastic inverse methods to estimate model parameters which cannot be described with a Gaussian distribution, e.g., hydraulic conductivities, integrating nonlinear model observations, e.g., hydraulic head data.

The first is the normal-score ensemble Kalman filter (NS-EnKF) constructed on the basis of the standard EnKF. The standard EnKF is widely used as a real time data assimilation technique due to its advantages, e.g., computation efficiency and ability to assess model uncertainty. However, it is known to perform optimally when the model parameters and state variables follow multiGaussian distributions. To extend the application of the EnKF to nonGaussian distributed state vectors, such as those in channelized fluvialdeposit aquifers, we introduce the normal-score transformation into the EnKF, to propose the NS-EnKF. The augmented state vector consisting of model parameters and state variables are normal-score transformed so that they follow marginal Gaussian distribution. Then, the transformed vectors serve as input to the EnKF, which now operates on marginally Gaussian distributed variables. The updated vectors are then back transformed to the original distribution space. The effectiveness of the proposed method is assessed in two synthetic bimodal aquifers, where the NS-EnKF is found to perform better than the standard EnKF in characterizing the bimodal structure of the hydraulic conductivities and in the subsequent flow and transport predictions.

The second method is a pattern search-based inverse method (PSINV), a novel inverse method that goes beyond the minimization of an objective function. The unknown model parameters are simulated by searching for pattern matches including parameters and state variables arranged in pre-established 
spatial templates. The attractive features of the proposed method, PSINV, include that (i) the estimated parameters do not necessarily follow a normal distribution, avoiding the pitfalls associated with the multiGaussian distribution, such as the lack of connectivity on extreme values; (ii) the model structures and the relationship between model parameter and state observations are characterized by multiple-point geostatistics, which renders it possible to apply the method in highly heterogeneous aquifers of complex geometries. The performance of the method is evaluated in two synthetic reservoirs composed of two facies, sand and shale. 


\section{Resumen}

La modelación numérica del flujo de agua subterránea y del transporte de masa se está convirtiendo en un criterio de referencia en la actualidad para la evaluación de recursos hídricos y la protección del medio ambiente. Para que las predicciones de los modelos sean fiables, estos deben de estar lo más próximo a la realidad que sea posible. Esta proximidad se adquiere con los métodos inversos, que persiguen la integración de los parámetros medidos y de los estados del sistema observados en la caracterización del acuífero. Se han propuesto varios métodos para resolver el problema inverso en las últimas décadas que se discuten en la tesis. El punto principal de esta tesis es proponer dos métodos inversos estocásticos para la estimación de los parámetros del modelo, cuando estos no se puede describir con una distribución gausiana, por ejemplo, las conductividades hidráulicas mediante la integración de observaciones del estado del sistema, que, en general, tendrán una relación no lineal con los parámetros, por ejemplo, las alturas piezométricas.

El primer método es el filtro de Kalman de conjuntos con transformación normal (NS-EnKF) construido sobre la base del filtro de Kalman de conjuntos estándar (EnKF). El EnKF es muy utilizado como una técnica de asimilación de datos en tiempo real debido a sus ventajas, como son la eficiencia y la capacidad de cómputo para evaluar la incertidumbre del modelo. Sin embargo, se sabe que este filtro sólo trabaja de manera óptima cuándo los parámetros del modelo y las variables de estado siguen distribuciones multigausianas. Para ampliar la aplicación del EnKF a vectores de estado no gausianos, tales como los de los acuíferos en formaciones fluvio-deltaicas, el NSEnKF propone aplicar una transformación gausiana univariada. El vector de estado aumentado formado por los parámetros del modelo y las variables de estado se transforman en variables con una distribución marginal gausiana. Estos vectores transformados sirven de entrada al EnKF, que ahora opera en variables cuya distribución marginal es gausiana. Los vectores actualizados resultantes acaban transformándose al espacio original. La eficacia del método propuesto se evalúa en dos acuíferos sintéticos con conductividades que siguen una distribución bimodal, donde el NS-EnKF produce mejores resultados que el EnKF en cuanto a la caracterización de la estructura bimodal de la conductividad hidráulica y las predicciones posteriores de flujo y transporte. 
El segundo método es un método inverso basado en patrones de búsqueda (PSINV), es un nuevo método inverso que va más allá de la minimización de una función objetivo. Los parámetros del modelo se simulan mediante la búsqueda de patrones en los que se incluyen parámetros y variables de estado dispuestos en plantillas preestablecidas. Las características mas atractivas del método propuesto, PSINV, son que (i) los parámetros estimados no tienen que seguir necesariamente una distribución normal, evitando los problemas asociados con la distribución multigausiana, tales como la falta de conectividad en los valores extremos, (ii) la heterogeneidad del modelo y la relación entre los parámetros del modelo y las variables de estado se modelan usando la geoestadística multipunto, lo que hace posible la aplicación del método en acuíferos altamente heterogéneo y con geometrías complejas. El método se evaluó en un depósito sintético compuesto de arena y pizarra. 


\section{Resum}

La modelitzaciò numèrica del flux d'aigua subterrània i del transport de massa s'està convertint en un criteri de referència en l'actualitat per a l'avaluació de recursos hídrics i la protecció del medi ambient. Perquè les prediccions dels models siguen fiables, aquests deuen estar el més pròxim a la realitat que siga possible. Aquesta proximitat s'obté amb els mètodes inversos, que persegueixen la integració dels paràmetres mesurats i dels estats del sistema observats en la caracterització de l'aqüífer. S'han proposat diversos mètodes per a resoldre el problema invers en les últimes dècades que es discuteixen en la tesi. El punt principal d'aquesta tesi és proposar dos mètodes inversos estocàstics per a l'estimació dels paràmetres del model, quan aquests no es poden descriure amb una distribució gausiana, per exemple, les conductivitats hidràuliques mitjançant la integració d'observacions de l'estat del sistema, que, en general, tindran una relació no lineal amb els paràmetres, per exemple, les altures piezomètriques.

El primer mètode és el filtre de Kalman de conjunts amb transformació normal (NS-EnKF) construït sobre la base del filtre de Kalman de conjunts estàndard (EnKF). El EnKF és molt utilitzat com una tècnica d'assimilació de dades en temps real a causa dels seus avantatges, com són l'eficiència i la capacitat de còmput per a avaluar la incertesa del model. No obstant açò, se sap que aquest filtre només treballa de manera òptima quan els paràmetres del model i les variables d'estat segueixen distribucions multigausianes. Per a ampliar l'aplicació de l'EnKF a vectors d'estat no gausians, tals com els dels aqǘfers en formacions fluvio-deltaiques, el NS-EnKF proposa aplicar una transformació gausiana univariada. El vector d'estat augmentat format pels paràmetres del model i les variables d'estat es transformen en variables amb una distribució marginal gausiana. Aquests vectors transformats serveixen d'entrada a l'EnKF, que ara opera en variables la distribució marginal dels quals és gausiana. Els vectors actualitzats resultants acaben transformantse a l'espai original. L'eficàcia del mètode proposat s'avalua en un aqǘfer sintètic amb conductivitats que segueixen una distribució bimodal, on el NSEnKF produeix millors resultats que el EnKF quant a la caracterització de l'estructura bimodal de la conductivitat hidràulica i les prediccions posteriors de flux i transport. 
El segon mètode és un mètode invers basat en patrons de recerca (PSINV), és un nou mètode invers que va més enllà de la minimització d'una funció objectiu. Els paràmetres del model se simulen mitjançant la recerca de patrons en els quals s'inclouen paràmetres i variables d'estat disposats en plantilles preestablides. Les característiques més atractives del mètode proposat, PSINV, són que (i) els paràmetres estimats no han de seguir necessàriament una distribució normal, evitant els problemes associats amb la distribució multigausiana, tals com la falta de connectivitat en els valors extrems, (ii) l'heterogeneïtat del model i la relació entre els paràmetres del model i les variables d'estat es modelen usant la geoestadística multipunt, la qual cosa fa possible l'aplicació del mètode en aqüifers altament heterogenis i amb geometries complexes. El mètode es va avaluar en un dipòsit sintètic compost d'arena i pissarra. 


\section{Acknowledgements}

I wish to thank my advisor Prof. J. Jaime Gómez-Hernández for allowing me studying for a $\mathrm{PhD}$ in the research group of hydrogeology, at Universitat Politècnica de València. He shows his wisdom and quality as an excellent advisor, he always has the capacity to explain a complicated problem with examples easy to understand and he is always ready to provide suggestions whenever I am stuck with some difficulties. I think I am lucky to have the opportunity to study under his instructions. Besides the academic guide, I would say Jaime is a good chef for cooking paella, a special food in Valencia, and I have enjoyed it so much.

The study is principally supported by China Scholarship Council (CSC No. [2007] 3020). Additional financial supports are provided by ENRESA (Project 0078000067), the European Commission (Projects FUNMIG and PAMINA) and the Ministry of Education, Spain. All of these financial aids are gratefully acknowledged.

I would like to thank Jianlin Fu at Stanford University for sharing his code on EnKF which serves as the basis of the new method proposed in the Chapter 3. Grégoire Mariethoz (University of New South Wales) and Philippe Renard (University of Neuchatel) are enthusiastic in answering questions about the Direct Sampling algorithm and I am very appreciated. Special thanks goes to Prof. Harrie-Jan Hendricks Franssen for providing me the opportunity to visit the research center Jülich, Germany. Much work in the Chapter 3 is finished there.

I also want to say thanks to all the professors and colleagues in the group of hydrogeology, Eduardo, Andres, Javier, Oscar, Pilar, Julio and Teng, also Fran, Gero and Salvador who have left the group. I have had a good time in Valencia with them. Thanks also goes to Teresa and the department secretaries for preparing various documents for me.

Last but not least, I will express gratitude to Liangping and our families who always take care of me and encourage me. 



\title{
Contents
}

\begin{abstract}
$\quad$ v
Resumen vii

Resum ix

Acknowledgements $\quad$ xi

List of Figures $\quad$ xvii

List of Tables $\quad$ xxiii

1 Introduction $\quad 1$

1.1 Motivation and objective . . . . . . . . . . . . . 1

1.2 Thesis organization . . . . . . . . . . . . . . 3
\end{abstract}

2 Inverse Methods in Hydrogeology: Trajectory and Future Trends (submitted to Journal of Hydrology) $\mathbf{5}$

Abstract ........................... 5

2.1 Introduction . . . . . . . . . . . . . . . 5

2.1.1 The forward problem and the inverse problem . . . . . 6

2.1.2 Why is the inverse problem necessary? . . . . . . . 7

2.1.3 Why is the inverse problem difficult? . . . . . . . 7

2.1.4 Outline of the paper . . . . . . . . . . . . 9

2.2 Evolution of inverse methods . . . . . . . . . . . . 10

2.2.1 Direct approach or indirect avenue? . . . . . . . . . . 10

2.2 .2 Linearization or not? . . . . . . . . . . . . . . . 11

2.2.3 Deterministic estimation or stochastic simulation? . . . 13

2.2.4 Minimization or sampling? . . . . . . . . . . . . . 16

2.2.5 Real time integration or not? . . . . . . . . . . . 19

2.2.6 Preserve prior structure or not? . . . . . . . . . . 22

2.2 .7 MultiGaussian or not? . . . . . . . . . . . 26

2.2.8 Trajectory of inverse approaches to date . . . . . . 27 
2.3 Recent trend of inverse methods . . . . . . . . . . . . . . 29

2.3.1 Integrating multi-sources information . . . . . . . . 29

2.3.2 Combining high-order moments . . . . . . . . . . . 30

2.3.3 Multiscale problem . . . . . . . . . . . . . . 30

2.4 Conclusions . . . . . . . . . . . . . . . . . . . 31

Bibliography. . . . . . . . . . . . . . . . 33

3 An Approach to Handling Non-Gaussianity of Parameters and State Variables in Ensemble Kalman Filtering (published in Advances in Water Resources)

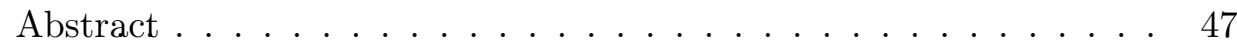

3.1 Introduction . . . . . . . . . . . . . . . 48

3.2 Methodology . . . . . . . . . . . . . . . . . 51

3.2.1 Flow and transport equations . . . . . . . . . . . 52

3.2 .2 Standard EnKF . . . . . . . . . . . . . . . 52

3.2.3 Normal-score EnKF . . . . . . . . . . . . . . . . . . 54

3.3 Synthetic experiment . . . . . . . . . . . . . 57

3.4 Performance assessment measures . . . . . . . . . . . . . . . 61

3.5 Results and discussion . . . . . . . . . . . . . . . 62

3.5.1 Standard EnKF versus NS-EnKF . . . . . . . . . . 62

3.5.2 Parameter sensitivity on NS-EnKF . . . . . . . . . 72

3.5.3 Discussion . . . . . . . . . . . . . . . . . 79

3.6 Conclusions . . . . . . . . . . . . . . . . . . 82

Bibliography. . . . . . . . . . . . . . . . 85

4 Pattern Recognition in a Bimodal Aquifer using the Normal-

Score Ensemble Kalman Filter (submitted to Mathematical Geosciences) 91

Abstract . . . . . . . . . . . . . . . . . . . . 91

4.1 Introduction . . . . . . . . . . . . . . . . . . . 92

4.2 The NS-EnKF . . . . . . . . . . . . . . . . . . 93

4.3 Synthetic example . . . . . . . . . . . . . . . . . 94

4.4 Results and discussions . . . . . . . . . . . . . . . . 97

4.4.1 Spatial variety characterization of $\operatorname{lnK} \ldots \ldots$. . . . . 97

4.4.2 Prediction capacity of updated $\ln K \ldots \ldots$. . . . 102

4.5 Conclusions . . . . . . . . . . . . . . . . . 105

Bibliography. . . . . . . . . . . . . . . . . . . 109

5 A Pattern Search Based Inverse Method (submitted to Water Resources Research) 113

Abstract . . . . . . . . . . . . . . . . . 113

5.1 Introduction . . . . . . . . . . . . . . . . . . . 114

5.2 Methodology . . . . . . . . . . . . . . 116 
5.2.1 Flow chart of the algorithm . . . . . . . . 116

5.2 .2 Distance function . . . . . . . . . . . . . . . . . . . . . . . . . 122

5.3 A synthetic example . . . . . . . . . . . . . . . . . . 123

5.4 Results and discussions . . . . . . . . . . . . . . . 125

5.4.1 Hydraulic conductivity characterization . . . . . . . 125

5.4.2 Prediction capability of the updated model . . . . . . . 127

5.5 A comparison test . . . . . . . . . . . . . . 131

5.5 .1 Reference . . . . . . . . . . . . . . 131

5.5.2 Prior model and conditioning data . . . . . . . . . 133

5.5.3 Calibrated model . . . . . . . . . . . . . . . . . 133

5.6 Conclusions . . . . . . . . . . . . . . . . . . . 135

Bibliography . . . . . . . . . . . . . . . . . . . . . 141

6 General Conclusions 147

6.1 Summary . . . . . . . . . . . . . . . . . . . . 147

6.2 Recommendations for future research . . . . . . . . . . . 149

$\begin{array}{ll}\text { Bibliography } & 151\end{array}$

$\begin{array}{ll}\text { Appendix } & 153\end{array}$

A Normal-score transform 153 


\section{List of Figures}

2.1 Schematic illustration of MLM, PiPM and SCM. Suppose the parameter to be estimated is the log hydraulic conductivity. The prior guess is updated by adding a perturbation, $\log \mathrm{K}_{\text {update }}$ $=\log \mathrm{K}_{\text {prior }}+\Delta \log \mathrm{K}$. The PiPM adds a perturbation around each pilot point sequentially. A seed logK field of the realization ensemble is shown for the SCM, which is modified by adding a perturbation that is computed by interpolating the perturbations at the master points. . . . . . . . . . 17

2.2 Workflow of the EnKF. . . . . . . . . . . . . . 22

2.3 Conceptual model of a fluvial deposited aquifer used as a training image by the multiple point based simulation algorithms. . 30

3.1 Flow chart of the NS-EnKF. . . . . . . . . . . . . 55

3.2 Training image used to generate the ensemble of binary facies

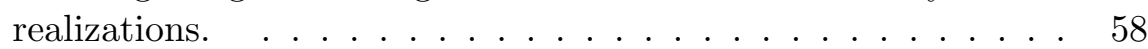

3.3 (A) Reference facies field; (B) reference log-conductivity field $(\ln (\mathrm{cm} / \mathrm{s}))$; (C) hydraulic conductivity measurements used for conditioning; (D) flow boundary conditions and location of piezometers (open circles for observation, solid circles for prediction);

(E) histogram of reference log-conductivity. . . . . . . . . . 59

3.4 A randomly selected $\ln K$ realization $\left(550^{\text {th }}\right)$ from the initial ensemble and the ensemble mean of all 1000 initial realizations for Scenarios 1 and $2 \ldots \ldots \ldots \ldots$

3.5 Configuration of the transport prediction experiment showing the line source where particles are injected and the control plane where breakthrough curves are measured (a few particle paths are shown for illustration). . . . . . . . . . . . .

3.6 The reference field together with a randomly selected realization $\left(550^{\text {th }}\right)$ of $\ln K$ and the ensemble mean of $\ln K$ for Scenarios 1 and 2 (standard EnKF and NS-EnKF) after 50 times steps of piezometric head data assimilation. 
3.7 Log-conductivity histograms for the initial ensemble of realizations and for Scenarios 1 and 2 (standard EnKF and NS-EnKF) after data assimilation. . . . . . . . . . . .

3.8 Probability that log-conductivity is larger than $-4 \ln (\mathrm{cm} / \mathrm{s})$ for the initial ensemble of realizations, and for the ensembles corresponding to Scenarios 1 and 2 (standard EnKF and NS-EnKF) after 50 times steps of piezometric head data assimilation. . .

3.9 Connectivity, as a function of the separation distance, measuring the probability that two points aligned in the $x$ direction are connected by a continuous path of log-conductivities larger than $-4 \ln (\mathrm{cm} / \mathrm{s})$. Gray curves correspond to individual realizations, their mean is given by the triangles (red) and the squares (black) correspond to the reference. Results are shown for the initial ensemble of realizations (conditioned on log-conductivity) and for Scenarios 1 and 2 (standard EnKF and NS-EnKF) after data assimilation. . . . . . . . . . . .

3.10 Piezometric head evolution at two of the thirty-two conditioning piezometers. Results are shown for the initial ensemble of realizations (conditioned only on log-conductivity) and for Scenarios 1 and 2 (standard EnKF and NS-EnKF, conditioned to transient piezometric heads up to 22.8 days). The vertical dashed lines indicate the conditioning period. Squares represent the piezometric head in the reference field. . . . . . . . . .

3.11 Piezometric head evolution at two of the eight validation piezometers. Results are shown for the initial ensemble of realizations (conditioned only on log-conductivity) and for Scenarios 1 and 2 (standard EnKF and NS-EnKF) after data assimilation. Squares represent the piezometric head in the reference field.

3.12 Summary of the breakthrough curves. The $5^{\text {th }}$ percentile, the median, and the $95^{\text {th }}$ percentile of the travel times are computed as a function of normalized concentration. Dashed lines correspond to the $5^{\text {th }}$ and $95^{\text {th }}$ percentiles, the solid line corresponds to the median, and the dotted line is the breakthrough curve in the reference. Results are shown for the initial ensemble of realizations (conditioned only on log-conductivity) and for Scenarios 1 and 2 (standard EnKF and NS-EnKF) after data assimilation. . . . . . . . . . . .

3.13 Evolution of log-conductivity ensemble mean as data are assimilated for time steps 1,10 and 50 (Scenario 2). . . . . .

3.14 Evolution of log-conductivity ensemble standard deviation as data are assimilated for time steps 1, 10 and 50 (Scenario 2). . 
3.15 The first three realizations and the ensemble mean before (left column) and after (right column) 50 times steps of piezometric head data assimilation using the NS-EnKF with an ensemble size of 200 realizations (Scenario 3). Notice how filter inbreeding effects the final results making all the realizations virtually the same. . . . . . . . . . . . . . . 7

3.16 A randomly selected realization $\left(550^{\text {th }}\right)$ and the ensemble mean before and after 50 time steps of piezometric head data assimilation using the NS-EnKF for the scenario where only 20 log-conductivity conditioning data are used (Scenario 4). . . . 78

3.17 New reference log-conductivity field with larger variance $(9.92$ $\left.\ln (\mathrm{cm} / \mathrm{s})^{2}\right)$ and corresponding histogram, together with a realization $\left(550^{\text {th }}\right)$ and the ensemble mean before and after 50 times steps of piezometric head data assimilation using the NS-EnKF

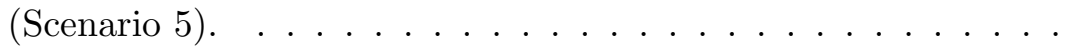

3.18 Summary of the breakthrough curves for Scenario 5. The $5^{\text {th }}$ percentile, the median, and the $95^{\text {th }}$ percentile of the travel times are computed as a function of normalized concentration. Results are shown for the initial ensemble of realizations (conditioned only on log-conductivity) and for the realizations after data assimilation. . . . . . . . . . . . . .

4.1 Training image used to generate the binary facies realizations (Strébelle, 2000). . . . . . . . . . . . . . .

4.2 Reference log-conductivity field and the histogram. Flow boundary conditions and locations of piezometers are also indicated.

4.3 Evolution of the $\ln K$ ensemble mean and variance as data are assimilated with the NS-EnKF for time steps 5, 15 and 60 . Reference $\ln K$ is shown for comparison. . . . . . . . . . . . 98

4.4 Evolution of the $\ln K$ ensemble mean and variance as data are assimilated with standard EnKF for time steps 5, 15 and 60 . Reference $\ln K$ is shown for comparison. . . . . . . . . . . 100

4.5 Evolution of Average Absolute Bias $\mathrm{AAB}(\ln K)$ and Average Ensemble Spread AESP $(\ln K)$ for sequential data assimilation with standard EnKF and the NS-EnKF. . . . . . . . . . . 101

4.6 $\ln K$ histograms for the (A) reference, (B) prior, (C) posterior with the standard EnKF, and (D) posterior with the NS-EnKF. 101 
4.7 Connectivity as a function of the separation distance, measuring the probability that two points in $x$ direction are connected by a continuous path of $\ln K$ larger than $0 \ln (\mathrm{m} / \mathrm{d})$. Shown are the results for $(\mathrm{A})$ the prior unconditional realizations, (B) the realizations conditioned with standard EnKF, and $(\mathrm{C})$ the realizations conditioned with the NS-EnKF. . . . . . . . . 103

4.8 Piezometric head evolution for two of the 111 piezometers, locations of which are indicated in Figure 4.2. Results are shown for the prior ensemble (no data assimilation), for assimilation with the standard EnKF and the NS-EnKF (conditioning to piezometric heads until 67.7 days, indicated by the vertical dashed line). Circles refer to piezometric head in the reference field.

4.9 Configuration of the transport prediction experiment. Shown are boundary conditions, locations for particle injection, location of the two control planes and an illustration of the particle

4.10 Breakthrough curves at two control planes for the prior ensemble and the ensemble updated with the standard EnKF and the NS-EnKF. The $5^{\text {th }}$ percentile, median, $95^{\text {th }}$ percentile and reference of the breakthrough curves are shown. . . . . . . 106

5.1 Flow chart of the proposed pattern-searching based multiplepoint ensemble inverse method. . . . . . . . . . 117

5.2 A pattern example consisting of conditional hydraulic conductivity and head data. $K_{i}$ is the value to be simulated. . . . . 119

5.3 Sketch map of the searching strategy. The dashed line indicates the exact location of $K_{i}$ through the ensemble. The candidates in the 3 by 3 square in each realization are evaluated to find the match consistent with the conditioning hydraulic conductivities and observed piezometric heads. . . . . . . . . . . . . 120

5.4 Sketch of filtering out noise. The black/white cells are converted to white/black so as to be consistent with the values in the neighborhood. . . . . . . . . . . . . 12

5.5 Training image used to generate the ensemble of binary facies realizations. . . . . . . . . . . . . . . . 124

5.6 Reference facies field. . . . . . . . . . . . . . . . . . . . . 124

5.7 The first four realizations in the ensemble. The left column shows four prior facies fields and the right column shows the corresponding updated facies. The reference field is also shown for comparison. . . . . . . . . . . . . . 126 
5.8 Ensemble average (the second row), standard deviation (the third row) and RMSE (the bottom row) of $\ln K$ over the ensemble before and after head data assimilation. The reference field (the top row) is also shown for comparison. . . . . . . . . 128

5.9 Piezometric head evolution at two conditioning piezometers, positions of which are shown in Figure 5.6. Results are shown for the prior ensemble and the updated ensemble. The dots represent the piezometric head in the reference field. . . . . . . . 129

5.10 Evolution of average $R M S E$ of piezometric heads over the field. 129

5.11 Ensemble average (the second row), Standard deviation (the third row) and RMSE (the bottom row) of hydraulic head over the ensemble before and after head data assimilation. Reference head field (the top row) is also shown for comparison. . . . . . 130

5.12 Configuration of the transport prediction experiment. . . . . 131

5.13 Summary of the breakthrough curves. The $5^{\text {th }}$ percentile, the median, and the $95^{\text {th }}$ percentile of the travel times are computed as a function of normalized concentration. Dashed lines correspond to the $5^{\text {th }}$ and $95^{\text {th }}$ percentiles, the solid line corresponds to the median, and the dotted line is the breakthrough curve in the reference. Results are shown for the prior ensemble and the updated ensemble. . . . . . . . . . . . . . . 132

5.14 Training image and reference field. (A) Training image (Strebelle, 2002). (B) Reference hydraulic conductivity field, in which the conductivities are measured at the 9 points serving as the hard data to generate the prior model and the piezometric head data at these wells are used to calibrate the prior model.

5.15 Comparison of the prior and calibrated model. A realization of the ensemble (the first row), probability of being sand (the second row) and variance (the third row). . . . . . . . . . 134

5.16 Hydraulic head at the end of simulation period in the reference field, prior model and updated model. Only one sample of the realization stack is shown. Hydraulic prediction uncertainty is assessed in the following figure. . . . . . . . . . . 136

5.17 Piezometric head evolution at the 9 conditioning piezometers, the positions of which are shown in Figure 5.14B. Results are shown for the prior ensemble (the first 9 plots) and the corresponding updated ensemble (the second 9). The dotted lines represent the piezometric head in the reference. Only the first 6 days were used as conditioning data. (to be continued) . . . 137

A.1 Sketch of normal-score transformation. . . . . . . . . . . 154 


\section{List of Tables}

2.1 Details of the inverse methods mentioned in the paper. The attributes of each inverse method are reported based on its most popular implementation. . . . . . . . . . . . 28

3.1 Scenario definition. Parameters that change from one scenario to another. . . . . . . . . . . . . . 57

3.2 Parameters defining the multiGaussian random function used to generate the log-conductivity within each of the two facies.

3.3 Comparison of the initial ensemble (only conditioned to conductivity data), standard EnKF and NS-EnKF (Scenarios 1 and 2 in Table 3.1, which are conditioned to both conductivity and piezometric head data). . . . . . . . . . . . 65

3.4 Performance measures for Scenarios 3,4 and $5 . \quad \ldots \ldots \ldots .77$

4.1 Parameters defining the multi-Gaussian random function used to generate the log-conductivity within each of the two facies. 



\section{1 \\ Introduction}

\subsection{Motivation and objective}

Numerical modeling prediction has become a common reference tool in groundwater management as well as for the remediation of contaminated sites. To construct a reliable model, it is indispensable to characterize as best as possible all the parameters and variables involved in the model. The intuitive method to obtain the parameters is in situ measurement from the field. The measured "hard data" (the term hard is used to indicate that it represents a direct measurement of the parameter of interest) are used to constrain the model parameters with a geostatistic algorithm, e.g., Deutsch and Journel (1998). However, it is in practice impossible to explore the aquifer exhaustively. Besides the mentioned hard data, we can take advantage of the "soft data" (the term soft is used to indicate that it represents an indirect measurement of the parameter of interest), which are collected easily and extensively, for instance, hydraulic head and remote sensing data, some of which can be integrated with the help of the geostatistic method while others have to be assimilated with the inverse method invoking the inverse problem.

The inverse problem aims at determining the unknown model parameters by integrating the observed model responses. However, the subsurface reservoir is normally very heterogeneous due to complex geologic process and physical and chemical reactions, which makes model parameter identification a demanding task. In the past several decades a series of inverse methods has been proposed to solve the inverse problem. Despite of different formulation 
details, most inverse methods estimate the parameters under the criterion of minimizing an objective function which measures the deviation between the simulated and observed data,

$$
J=\Phi\left(Y(x)^{s i m}, Y^{o b s}\right)
$$

where $Y(x)^{\text {sim }}$ is simulated model states, $x$ represents the parameters to be estimated and $Y^{o b s}$ the observations. A first problem that arises if we focus only on the state reproduction is that the identified model structure might be geologically unrealistic (Kitanidis, 2007). A solution to this problem is characterizing correctly the prior structure and preserving the prior statistics during the inverse modeling.

In the conventional inverse problem the aquifer structures are characterized by a two-point based variogram model which evaluates correlation between two locations. The variogram model is deficient to describe curvilinear structures exhibited by some complex subsurface reservoirs, e.g., fluvial deposit aquifers. An approach to handle the nonlinear structures is multiple-point geostatistics (Guardiano and Srivastava, 1993), in which a training image is introduced serving as the conceptual model. The high order statistics, experimental local conditional distribution function and structure patterns, are derived from the training image and transferred to the generated realizations.

Field data indicate that the model parameters, e.g., hydraulic conductivity, display a log-normal histogram. By extension, many researchers have assumed that the best statistical model to characterize the spatial variability of logconductivity is the multiGaussian distribution. The multiGaussian distribution is attractive since it is fully described by two values, expectation and covariance. Some inverse methods are known to work optimally in a multiGaussian framework. For instance, the ensemble Kalman filter (EnKF) provides an optimal solution when the parameters and state variables follow a multiGaussian distribution (Arulampalam et al., 2002). However, the appropriateness of the multiGaussian distribution has been challenged for some decades now (e.g., Gómez-Hernández and Wen, 1998; Journel and Deutsch, 1993) and the multiGaussian model has been found unreasonable in such cases that there exist continuous paths composed of extreme high (low) values, or for intricate spatial heterogeneity patterns. Some inverse methods are thus proposed to go beyond the limitation of the multiGaussian distribution, e.g., particle filtering.

The objective of this thesis is to propose inverse methods coping with the above mentioned problems, that is, the new inverse methods are supposed to satisfy the standards listed below: (a) preserve a prior model structure to ensure geological realism; (b) perform well even if the model parameters do not follow a multiGaussian distribution; (c) characterize the curvilinear structures in complex aquifers which cannot be described with a two-point variogrambased model; (d) develop some optimization scheme which goes beyond the 
objective function framework. Contribution of this thesis includes two novel inverse methods, normal score EnKF (NS-EnKF) and a pattern search based inverse method (PSINV). The former is constructed by modifying the standard EnKF so that it can handle nonGaussian distributed model parameters and states and the latter is a pattern searching based inverse method dealing with categorical variables in a multimodal reservoir based on multiple point geostatistics.

Hereafter, notice that we will use interchangeably the terms data assimilation, inverse modeling and conditioning, they have the same meaning and are commonly used in filtering, hydrogeology and geostatistics, respectively to mean that we aim at generating realizations of a parameter honoring the measured data.

\subsection{Thesis organization}

The rest of the thesis is organized as follows:

Chapter 2 recalls the evolution of the inverse modeling ranging from the preliminary manual calibration to recent automatic data assimilation methods. Shortcomings and advantages of each type of inverse method are highlighted, in which the shortcomings will serve as the motivation for developing new methods and advantages will be retained when developing new techniques. Recent trends of the inverse problem are also discussed in this chapter.

Chapter 3 modifies the standard EnKF so that it can deal with nonGaussian distributed model parameters and model states. It is well known that the standard EnKF performs optimally when the parameter follows a Gaussian distribution and the model transfer function is linear. In this chapter, the parameters and state variables are transformed via normal-score transformations so that they follow a marginal Gaussian distribution. The Kalman filtering is then carried out in the Gaussian space. The NS-EnKF is compared with the standard EnKF to assess its performance through a synthetic example which resembles a channelized fluvial deposit aquifer. Furthermore, a sensitivity analysis of the NS-EnKF is presented.

Chapter 4 evaluates the performance of NS-EnKF under the stringent condition that no parameter measurements (hard data) are available. In this experiment, the hydraulic conductivities of a bimodal subsurface reservoir are estimated by incorporating piezometric head observations.

Chapter 5 proposes a novel inverse method, a pattern search-based inverse method and the program code, PSINV. The model parameters are simulated by pattern matching of pre-defined templates composed of a combination of parameter and state variable values rather than by minimizing an objective function. The multiple-point based pattern matching allows identifying curvi- 
linear structures impossible to capture with a two-point variogram model. The performance of PSINV is illustrated in a bimodal facies reservoir composed of sand and shale.

The above four chapters are in fact four independent papers which have been published, are under review or to be submitted.

Chapter 6 summarizes the thesis and gives recommendations for future research.

Appendix A shows in detail how the transformation function needed in the NS-EnKF is constructed including normal score transform and back transform. 
Journal of Hydrology, submitted.

\title{
2 \\ Inverse Methods in Hydrogeology: Trajectory and Future Trends
}

\begin{abstract}
Parameter identification is an essential step in constructing a groundwater model. The process of recognizing model parameters by conditioning on the observed data is inverse problem. A series of inverse methods has been proposed to solve the inverse problem, ranging from trial-and-error manual calibration to the current complex automatic data assimilation algorithms. This paper does not attempt to be another review paper on inverse models, but rather to analyze and track the trajectory of the inverse methods over the last decades, revealing their evolution, motivation and future trends. Issues confronted by the inverse problem, such as dealing with multiGaussianity or whether to preserve or not the prior statistics are discussed.
\end{abstract}

\subsection{Introduction}

Mathematical modeling of subsurface flow and mass transport is needed for groundwater resources management and contamination remediation. The forward model requires specification of a variety of parameters, for instance, hydraulic conductivity, storativity and source or sink together with initial and 
boundary conditions. However, in practice it is impossible to characterize the model exhaustively because of the complex hydrogeological environment; for this reason, inverse modeling is indispensable. Inverse models are used to identify input parameters at unsampled locations by incorporating observed model responses, e.g., hydraulic conductivities are derived after hydraulic head and/or solute concentration data are combined. Deriving model parameters from model state observation is common in many other disciplines, such as petroleum engineering, meteorology and oceanography. This work mostly focuses on inverse methods used in hydrogeology.

\subsubsection{The forward problem and the inverse problem}

The forward problem involves predicting model states, e.g., hydraulic head, drawdown and solute concentration, based on a prior model parameterization. Combining the mass conservation and Darcy's laws, the forward groundwater flow model in an aquifer can be written as (Bear, 1972)

$$
\nabla \cdot(K \nabla h)=S_{s} \frac{\partial h}{\partial t}+Q
$$

subject to initial and boundary conditions, where $\nabla$. is the divergence operator $\left(\frac{\partial}{\partial x}+\frac{\partial}{\partial y}+\frac{\partial}{\partial z}\right), \nabla$ is the gradient operator $\left(\frac{\partial}{\partial x}, \frac{\partial}{\partial y}, \frac{\partial}{\partial z}\right)^{\mathrm{T}}, K$ is hydraulic conductivity $\left[L T^{-1}\right], h$ represents hydraulic head $[L], S_{s}$ is specific storage $\left[L^{-1}\right], t$ is time $[T]$, and $Q$ is source or sink $\left[T^{-1}\right]$. The differential equation governing non-reactive transport in the subsurface is:

$$
\phi \frac{\partial C}{\partial t}=-\nabla \cdot(q C)+\nabla \cdot(\phi \mathrm{D} \nabla C)
$$

subject to initial and boundary conditions, where $C$ is the concentration of solute in the liquid phase $\left[M L^{-3}\right], \phi$ is porosity [-], D is the local hydrodynamic dispersion coefficient tensor $\left[L^{2} T^{-1}\right]$, and $q$ is the Darcy velocity $\left[L T^{-1}\right]$ calculated by Darcy's law: $q=-K \nabla h$.

The inverse problem aims at determining the unknown model parameters by making use of the observed state data. In the early days of groundwater modeling, it was common to start with a prior guess of the model parameters, run the forward model to obtain the simulated states, and then enter in a manual loop of comparison between simulated and observed states, modification of parameters, and forward model running, until observed and simulated values were close enough so as to accept the model parameter distribution as a good representation of the aquifer. This "trial and error" method falls into the scope of "indirect methods" as opposed to the "direct methods" which do not require multiple runs of the forward model to derive the model parameters (Neuman, 1973) as will be discussed below. 


\subsubsection{Why is the inverse problem necessary?}

Sagar et al. (1975) classified the inverse problem into five types according to the unknowns, i.e., model parameters, initial conditions, boundary conditions, sources or sinks and a mixture of the above, respectively. Most documented inverse methods fall into the first type, that is, they try to identify model parameters which contribute a large amount to model uncertainty due to the inherent heterogeneity of aquifer properties. Parameter identification is of importance considering the fact that no reliable predictions can be acquired without a good estimation of model parameters. Besides estimation of aquifer parameters, inverse methods are also able to provide an assessment of uncertainty for the predictions. Furthermore, inverse problem might serve as a guide for data collection and the design of an observation network. The reader is referred to Poeter and Hill (1997) who discussed the benefits of inverse modeling in depth.

\subsubsection{Why is the inverse problem difficult?}

A problem is properly posed if the solution exists uniquely and varies continuously as the input data changes smoothly. However, most of the inverse problems in hydrogeology are ill-posed and they cannot be solved unless under certain assumptions and constraints. Ill-posedness may give rise to three problems: non-uniqueness, non-existence and non-steadiness of the solutions, among which non-uniqueness is the most common. Non-uniqueness primarily stems from the fact that the number of parameters to be estimated exceeds that of the available observation data. Another reason is that the parameters to be identified are sometimes not sensitive to the observations; for instance, hydraulic conductivities are not as sensitive to head data close to prescribed head boundaries as they are to head data close to prescribed flux boundaries (Carrera and Neuman, 1986b).

A series of suggestions have been proposed to alleviate the ill-posedness:

1. Reduce the number of unknown parameters, e.g., using zonation, or collect more observation data so that the numbers of data and unknowns are balanced.

2. Consider the prior information or some other type of constraint so as to restrict the space within which parameters may vary.

3. Impose regularization terms to reduce fluctuations during the optimization iterations.

4. Maximize the sensitivity of heads to model parameters, for instance, by designing properly the observation network. 
5. Minimize the nonlinearity in the model equation. Carrera and Neuman (1986b) argued that working with the logarihtm of hydraulic conductivity reduces the degree of non-convexity during optimization. An alternative is to infer hydraulic conductivity using fluxes rather than heads as done by Ferraresi et al. (1996), since the relationship between hydraulic conductivity and flux is linear (Darcy's law) while the relationship between hydraulic conductivity and head is nonlinear.

Detailed discussions can be found in works by Emsellem and De Marsily (1971), Neuman (1973), Carrera and Neuman (1986b) and McLaughlin and Townley (1996) among others.

Besides the ill-posedness problem, computational burden is the second main hurdle for inverse problems (Neuman, 2006). There are several reasons for the high CPU time requirement. Since many inverse models are iterative, the forward model has to be run many times until an acceptable parameter distribution is obtained. The time needed to run the forward model grows exponentially with the degree of discretization and the level of heterogeneity. When multiple realizations are sought, CPU demand grows with the number of realizations. For those methods that require them, computing sensitivity matrices is very time consuming. A few measures to reduce computational demand have been proposed, for instance, (a) certain kernel technique renders it possible to select representative realizations from a large ensemble so that the size of the ensemble can be reduced (Scheidt and Caers, 2009); (b) scale-up can be taken prior to any forward simulation in order to reduce solution time (e.g., Li et al., 2011d); (c) some improvements have been reported to speed up the calculation of the sensitivity Jacobian matrices (Medina and Carrera, 2003).

The problem of scales is the third difficulty to be confronted in the application of the inverse method. Measurements from boreholes (made in situ or in the laboratory), local pumping tests, and regional aquifer estimates are the three common scales (Dagan, 1986; Kitanidis and Vomvoris, 1983) at which information is handled in aquifer modeling. As Emsellem and De Marsily (1971) pointed out, "permeability is a parameter with no punctual value but with an average value in a region of a given size". The support of the permeability measured from the field is normally smaller than the cell size of the numerical model. In practice, permeability should be upscaled to a scale consistent with that of the numerical model discretization, otherwise the forward model would be computationally very expensive. A variety of approaches to calculate the upscaled permeability or hydraulic conductivity are available (e.g., Renard and Marsily, 1997; Sanchez-Vila et al., 2006; Wen and Gómez-Hernández, 1996; Zhou et al., 2010; Li et al., 2011a,b). Besides the scale inconsistence between the field measurement support and numerical model discretization, 
another scale problem is related with variety of observations, i.e., the observed data may be obtained in different supports.

\subsubsection{Outline of the paper}

Despite all sorts of difficulties, many inverse methods have been proposed to solve the inverse problem. In the present paper we do not intend to review all current inverse methods, since several others have reviewed the topic from different points of view (e.g., Carrera et al., 2005; De Marsily et al., 2000; McLaughlin and Townley, 1996; Oliver and Chen, 2010; Yeh, 1986). But rather, we would like to analyze the evolution of the inverse models, from the simple trial-and-error approaches of yesterday to the sophisticated ensemble Kalman filter of today, pointing out the incremental improvements that happened in the way. In the remainder of this paper, we mainly illuminate seven key topics as follows.

- Section 2.2.1 discusses the direct method. We focus on the indirect methods in the following sections.

- Section 2.2.2 shows a linear inverse method in which the groundwater flow model is solved by linearizing the partial differential equation under certain assumptions. Its shortcoming motivates development of the nonlinear inverse methods in which the forward problem is solved numerically. The inverse methods in the rest sections all belong to the nonlinear type.

- Section 2.2.3 highlights the importance of considering uncertainty and introduces the inverse method based on Monte Carlo simulation in which multiple plausible realizations are used to represent the real system.

- Section 2.2.4 displays another way to update the model, i.e., by sampling the posterior distribution rather than by minimizing an objective function which measures the mismatch between the simulated and observed data.

- Section 2.2.5 focuses on integrating new observations sequentially without the need to reformulate the problem.

- Section 2.2.6 talks about whether the prior statistical structure of the model should be preserved.

- Section 2.2.7 addresses the issue of multiGaussianity in inverse modeling and the difficulties to get away from it. 
In each section, we will introduce a typical inverse method explaining its principle, implementation details, advantages and shortcomings. Recent trends of the inverse problem are summarized in Section 2.3. The paper ends with some conclusions.

\subsection{Evolution of inverse methods}

Many approaches have been proposed to solve the inverse problem. Several comparison studies have been carried out to evaluate their performances, among them Zimmerman et al. (1998) and Hendricks Franssen et al. (2009) both compared seven different inverse methods. The former work focused on the transmissivity estimation and subsequent forecast of transport at the Waste Isolation Pilot Plant (WIPP). The latter applied seven inverse methods to characterize the well catchments by groundwater flow modeling. Some methods, such as the maximum likelihood method, the self-calibration method and the pilot point method were analyzed in both works. In this section we would like to track the evolution of inverse methods under different driving forces.

\subsubsection{Direct approach or indirect avenue?}

Inverse methods fall into two groups: direct and indirect ones (Neuman, 1973). Nowadays, only indirect methods are considered; however, it was the direct method that, somehow, gave rise to the indirect one. The move from direct methods to indirect ones would be the first major evolution in solving inverse problem, and for this reason we start this analysis by recalling the direct method and why it had to be discarded in favor of the indirect one.

The forward problem of groundwater flow model can be expressed generally as $F(K)=h$, where $F(\cdot)$ is the equation (e.g., Equation 2.1) relating the system parameters (e.g., hydraulic conductivity $K$ ) to the model response (e.g., hydraulic head $h$ ). The inverse problem can be simply formulated as the inverse: $K=F^{-1}(h)$, with the known responses but unknown parameters. The boundary conditions and sources or sinks terms could also be identified if necessary (Sagar et al., 1975).

Although the theory is straightforward, it is virtually impossible to obtain realistic solution by solving the algebraic equation series due to the serious ill-posedness or the singularity of the matrices involved in the numerical formulation of the problem (Sun, 1994). Some modifications were proposed to cope with the difficulties, such as: consider more equations than there are unknowns to build an over-determined system so that the effect of measurement errors is reduced (Ponzini and Lozej, 1982); or, impose a constraint on the 
objective function, which converts the inverse problem into a linear programming (Kleinecke, 1971; Neuman, 1973) or a minimization problem (Navarro, 1977).

The main shortcoming of the direct method is that it requires that piezometer heads have been measured at all nodes of the discretized domain, and for it to yield stable results head measurements are needed everywhere for several orthogonal boundary conditions in the sense explained by Ponzini and Lozej (1982).

It is worthwhile mentioning that, recently, Brouwer et al. (2008) proposed a direct approach called the "double constraint method" to determine permeability. Although it is not strictly a direct method, since it does not require having observations extensively over the entire domain, the final step of the method, computing permeabilities, is a direct approach. The methods assumes that couples of pressure/flow rates are available at a number of points in the domain. A guess of the spatial distribution of the permeabilities is made and two forward runs are performed, the first one considers the measured pressures as prescribed boundary conditions (disregarding the flow rate data), the second one uses the flow rates as prescribed boundary conditions (disregarding the pressure data), then prior permeability guess is forgotten and new permeabilities are computed "directly" at each block interface using the pressure gradients derived from the first run and the flow rates derived from the second one. The process is repeated, and eventually the spatial distribution of permeabilities converges to a stable one. The method was compared in a synthetic example with the results obtained by the ensemble Kalman filter yielding good results.

The virtual impossibility of having state observation data on the entire domain gave rise to the indirect methods capable of handling limited numbers of observations.

\subsubsection{Linearization or not?}

Kitanidis and Vomvoris (1983) proposed the geostatistical approach (GA) as a very clever way to reduce the number of unknown values and subsequently mitigate the ill-posedness problem. Using geostatistical tools, identifying the conductivities is not performed by trying to guess the values of conductivity in each node of the aquifer domain, but rather by determining the parameters of the random function model underlying such a spatial distribution; then, conductivities are interpolated by kriging onto the model cells.

The procedure of the GA can be summarized into two main parts: structure analysis and linear estimation. Structure analysis consists of three steps as follows: 
1. Select a geostatistical model, e.g., decide a variogram function and whether the model is stationarity or not. The model structure is selected based on all available information including hydraulic properties.

2. Estimate the parameters characterizing the model structure such as trend (if any), variance and correlation ranges. The joint probability function of log-permeability and head is assumed multiGaussian, and the hydraulic heads are expressed as a linear function of log-permeability, after linearizing Equation 2.1. Then, the parameters of the geostatistical model (generally no more than five) are estimated through maximum likelihood. Gauss-Newton method is used to solve the iterative maximization of the likelihood function.

3. Examine the validity of model. The estimated structure is either accepted or modified during the test (i.e., the variogram function is changed, or anisotropy is introduced).

As soon as the geostatistical model is accepted, the log-permeability field is estimated by kriging, a best linear unbiased estimation algorithm, which is capable of providing estimations with minimum error variance. Later, Dagan (1985) and Rubin and Dagan (1987) proposed an extension of the GA using Bayesian conditional probabilities.

The advantages of the GA reside in two main aspects. First, it reduces the number of the effective parameters to be estimated by introducing the concept of random function into the inverse problem. In this way, the ill-posedness is alleviated since the unknown value number is far less than the number of observations and the estimated parameters are independent of grid discretization. Second, this method is computationally efficient arising from two facts: hydraulic head is obtained by first-order approximating the flow equation instead of numerical solving it; a linear estimation (kriging) is applied as soon as the geostatistical structure is identified with no iterative optimization involved, saving CPU time to a large extent. The method was first verified through a one-dimensional test and found stable and well-behaved (Kitanidis and Vomvoris, 1983).

Despite several advantages of this method, we have to mention some shortcomings. First, the hydraulic head in the forward problem is approximated by linearizing the flow equation under an assumption which is only valid if the log-conductivity exhibits a small variance. Hoeksema and Kitanidis (1984) alleviated this problem by applying the method to a two-dimensional isotropic confined aquifer in which hydraulic heads are obtained by solving numerically the partial differential equation, and then Kitanidis (1995) further generalized it onto a quasi-linear approach. Second, the final conductivity map is obtained by kriging, this has two implications: first, and most important, the 
final maps are smooth since they represent an ensemble expected value of the random function model, and second, since kriging only uses the covariances for the estimation, as soon as heads cannot be approximated as a linear combination of the conductivities, the final conductivity maps do not honor the measured piezometric heads, in the sense that the forward solution of the flow problem will not match the measured heads.

The need to apply the inverse model to hydraulic conductivity spatial distributions with large heterogeneity forced moving from the linearized approaches to other approaches in which this linearization is not necessary.

\subsubsection{Deterministic estimation or stochastic simulation?}

A typical example of nonlinear inverse approaches is the maximum likelihood method (MLM) developed by Carrera and Neuman (1986a). The method is able to estimate simultaneously such parameters as hydraulic conductivity, specific storage, porosity, dispersivity, recharge, leakage and boundary conditions by incorporating head and concentration measurements as well as prior information (Medina and Carrera, 1996). Zonation is employed to reduce the number of parameters to be estimated, that is, hydraulic conductivities are assumed constant over large patches of the aquifer, thus, the number of unknowns is proportional to the number of patches. Then, the groundwater problem (Equation 2.1, or 2.2 or both) is solved numerically subject to initial and boundary conditions. Let $x$ be a vector of all the unknown parameters and $y^{o b s}$ be a vector of available measurements, a set of optimum parameter estimates is obtained by maximizing the likelihood $L\left(x \mid y^{\text {obs }}\right)$. Under the hypothesis that all the data could be transformed to jointly follow a multiGaussian distribution, the likelihood function can be expressed as follows:

$$
L\left(x \mid y^{o b s}\right) \propto \exp \left\{-\frac{1}{2} \sum_{i=1}^{N_{m}}\left(y_{i}-y_{i}^{o b s}\right)^{\mathrm{T}} C_{y, i}^{-1}\left(y_{i}-y_{i}^{o b s}\right)\right\}
$$

where $y_{i}$ can be computed states (e.g., head and concentration) and model parameters, $C_{y, i}$ is the corresponding covariance of $y_{i}$ and $N_{m}$ is the number of types of measurements. Maximizing $L\left(x \mid y^{o b s}\right)$ is equivalent to minimize $-2 \ln (L)$, and the optimization problem turns to minimizing the objective function:

$$
J=\sum_{i=1}^{N_{m}}\left(y_{i}-y_{i}^{o b s}\right)^{\mathrm{T}} C_{y, i}^{-1}\left(y_{i}-y_{i}^{o b s}\right) .
$$

Iterative minimization algorithms are applied on the objective function until certain convergence criteria are met. The model parameters are then updated by adding the optimal perturbations. The uncertainty of parameter estimates is evaluated by a lower bound of the covariance matrix. 
One of the important features of MLM is that the number of unknown parameters is reduced significantly due to the application of zonation so that the potential ill-posedness problem is circumvented to some extent. Furthermore, the MLM might be used as a conceptual model identification tool, i.e., to identify the best model structure among several alternatives; in this respect, Carrera (1987) argued that the criterion proposed by Kashyap (1982) is the most effective.

However, some limitations of the MLM are apparent. Although the zonation scheme does help to reduce the number of the unknowns, it causes over smoothness, i.e., inherently heterogeneous geologic properties are represented by a few patches of homogeneous conductivities, while at the same time may introduce unacceptable discontinuities between zones. Furthermore, some zone discretizations may cause bias, depending on the number of zones and measurements (Kitanidis, 1996). Also, as stated earlier, the objective function is constructed under the assumption of a multinormal distribution, i.e., the prior residuals and estimates follow a multiGaussian distribution. The implications of this assumption will be discussed further in Section 2.2.7.

The MLM was probably the first widely successful inverse method, it could incorporate many types of observations, it included a regularization term to prevent wide fluctuations during the optimization phase, and, because it did not use any approximation for the relationship between the state variables and the aquifer parameters, it yielded a zoned map of hydraulic conductivities that reproduced very well the observed data. However, the MLM method produced a single map, too smooth to really describe the heterogeneity observed in nature.

Small scale variability had already being recognized as one of the important factors controlling aquifer response. Recognizing this, De Marsily et al. (1984) introduced the pilot point method (PiPM) as a procedure to introduce more variability in the aquifer model. Starting from a kriging map of the hydraulic conductivities, smooth as all kriging estimation maps are, De Marsily et al. (1984) positions a fictitious datum where no observation exists, and assigns to it a value so that when kriging is performed again, with this new datum, the new kriging map provides a better approximation to the observed piezometric head data. New pilot points are introduced sequentially into the model until there is enough heterogeneity in the model so as to reproduce the observed head data. This procedure had several advantages: the aquifer could be discretized at any scale, since after each iteration, (block) kriging was performed on the entire aquifer; the heterogenity was consistently treated throughout the process, since the same variogram model was used for all kriging estimations; and, because of the way the procedure is implemented, the fictitious data introduced at the pilot points was always within the local limits of variability of the variable as induced by the underlying random function model. The PiPM 
was successfully applied to model the Culebra formation overlying the Waste Isolation Pilot Plan (WIPP) (Cooley and Hill, 2000; LaVenue et al., 1995; RamaRao et al., 1995, 2000). The main problem of the PiPM was still that, at the end, there was only a single representation of the aquifer, a single kriging map that, although more heterogeneous than the kriging map computed from the conductivity data alone, still was too smooth.

An estimated map, let it be obtained by kriging, maximum likelihood or Bayesian conditional probabilities, it is an average map, and the average does not necessarily represent reality. The same way that the average outcome of throwing a dice (3.5) does not correspond to any of the dice face pips, the average of an ensemble of potential realizations, does not correspond with any realization. The smooth fields obtained by these methods fail to reflect the local spatial variability and will necessarily fail in properly predicting mass transport (e.g., Gómez-Hernández and Wen, 1994).

It is then when the self-calibrated method is proposed (SCM) and the concept of inverse stochastic modeling starts being considered. The idea is not to seek a single smooth representation of the spatial variability of hydraulic conductivity capable of reproducing the observed piezometric head and/or concentration data, but to generate multiple realizations, all of which displaying realistic patterns of short scale variability, all of which reproducing the observed piezometric head and/or concentration data. The concept of the SCM was first outlined by Sahuquillo et al. (1992) and then elaborated by Gómez-Hernández et al. (1997) accompanied with two applications and an implementation program (Capilla et al., 1997, 1998; Wen et al., 1999). The SCM is based on the PiPM with the following rationale: instead of starting from a kriging map and introducing local perturbations by adding fictitious pilot points and optimizing the value that would produce the best reproduction of the observed state, let's start from multiple realizations generated by a conditional simulation algorithm; and instead of identifying the optimal location of the next pilot point and introducing them sequentially, locate what Gómez-Hernández et al. (1997) call master points all at once (as many as two or three per correlation length) and determine the values of the perturbations in a single optimization step. To understand the SCM, one has to recall that a conditional realization is the sum of a conditional mean (kriging map) plus a correlated residual map (Journel, 1974), what SCM is to apply the pilot point method with multiple points at once to modify the conditional mean with the objective that the new conditional mean plus the correlated residuals would match the observation data. By applying this optimization approach to all the realizations of an ensemble, the SCM is capable to generate multiple realizations, all of which are conditional to the state observation; thus, the term inverse stochastic modeling. 
With such an ensemble of realizations, it was possible to make transport predictions in each of the realizations and collect all of these predictions to build a model of uncertainty based on transport predictions made on realistic realizations.

It was soon realized that the concept of the SCM could be implemented in the original PiPM, and it has been applied multiple times since then (e.g., Alcolea et al., 2006, 2008; Lavenue and De Marsily, 2001).

Gómez-Hernández et al. (2003) reviewed stochastic conditional inverse modeling showing the strengths of SCM. The SCM was extended to incorporate concentration data (Hendricks Franssen et al., 2003; Wen et al., 2002) for the characterization of hydraulic conductivity, and also to incorporate breakthrough data of both reactive and nonreactive data to characterize the spatial variability of the sorption coefficient (Huang et al., 2004). Recently, genetic algorithms have been coupled with the SCM to search for the optimal locations of the master points as well as the optimal perturbation at these locations (Wen et al., 2006).

Both the PiPM and the SCM have been highly criticized recently by Rubin et al. (2010) who present as an alternative the anchored distribution method.

The MLM, the PiPM and the SCM are closely related in that they follow a very similar perturbation and updating scheme. The update process for the PiPM is illustrated in "Figure 1" of RamaRao et al. (1995) and in "Figure 1" of Alcolea et al. (2006). For the sake of completeness and comparison, we graphically show an sketch of the update algorithm for all the three methods (Figure 2.1). In the MLM, it is like that there is a pilot point in each zone, and the perturbation in the pilot point extends as a constant over the entire zone; in the PiPM, the pilot point perturbation dies out with the correlation length of the random function model; and in the SCM, the perturbation of the entire field is obtained by kriging the perturbations in each master point. All three methods seek finding those perturbations that added to the initial guess will result in a new field that is conditional to the observed state data.

\subsubsection{Minimization or sampling?}

Up to here all inverse methods discussed are based on the minimization of an objective function that measures the mismatch between the simulated state and the observed values. However, there are alternative ways to achieve the same results without resorting to an optimization problem, but rather to sampling a multivariate probability distribution.

Suppose that model parameter $x$ is characterized by a multiGaussian distribution with mean $\mu$ and variance $C_{x}, x \sim N\left(\mu, C_{x}\right)$, with a prior probability 


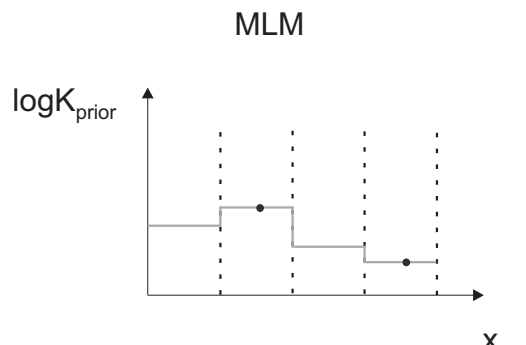

PiPM

SCM
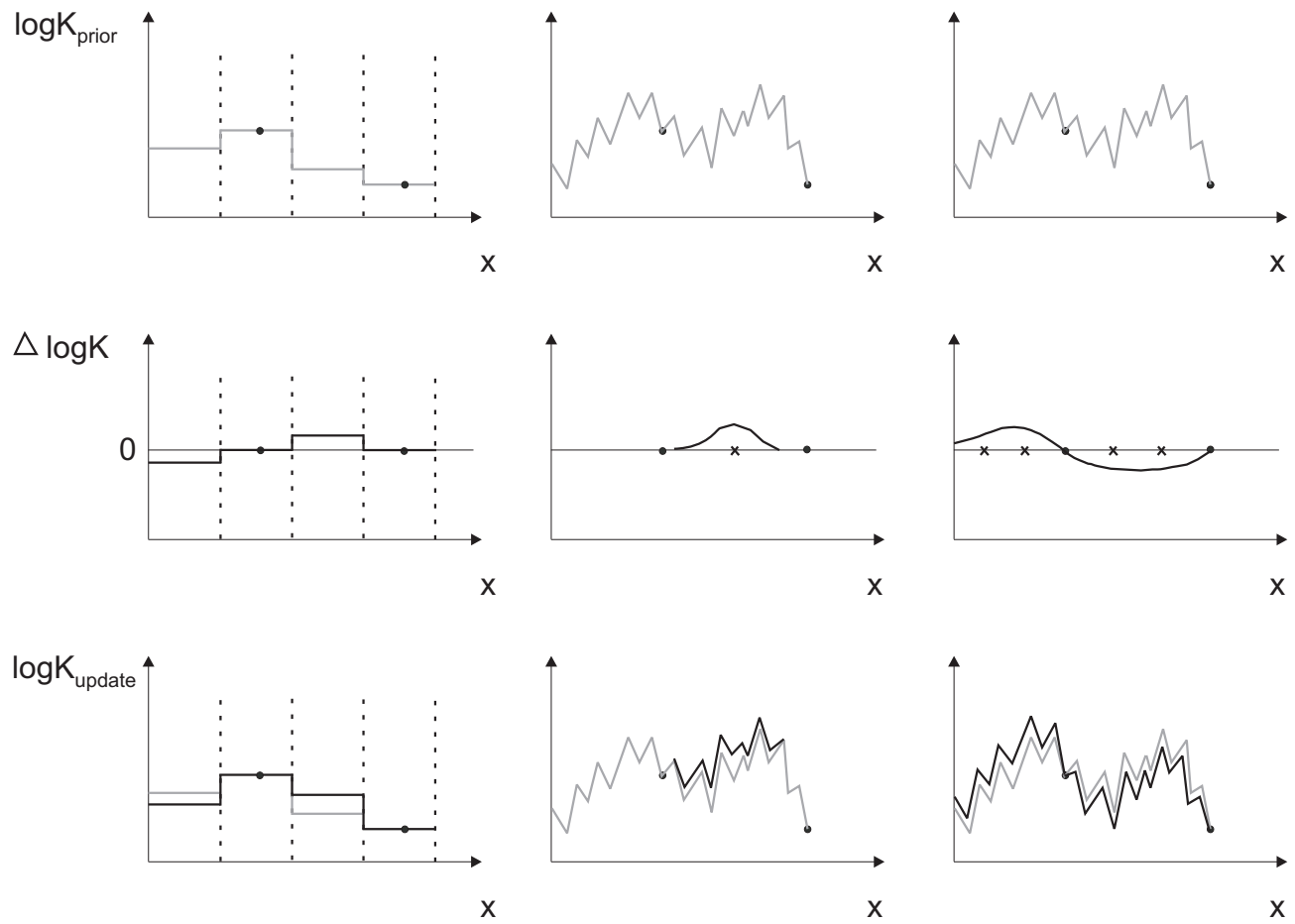

- Measurement

$\times \quad$ Pilot point or master point

Figure 2.1: Schematic illustration of MLM, PiPM and SCM. Suppose the parameter to be estimated is the log hydraulic conductivity. The prior guess is updated by adding a perturbation, $\log \mathrm{K}_{\text {update }}=\log \mathrm{K}_{\text {prior }}+\Delta \operatorname{logK}$. The PiPM adds a perturbation around each pilot point sequentially. A seed $\log \mathrm{K}$ field of the realization ensemble is shown for the SCM, which is modified by adding a perturbation that is computed by interpolating the perturbations at the master points. 
density given by

$$
\pi(x) \propto \exp \left\{-\frac{1}{2}(x-\mu)^{T} C_{x}^{-1}(x-\mu)\right\} .
$$

Assuming that the discrepancies between observed state variables $y^{\text {obs }}$ and their corresponding model simulations $y^{\text {sim }}=F(x)$ is also multiGaussian with error covariance $C_{y}$, the joint probability distribution of $y^{o b s}$ given $x$ is,

$$
\pi\left(y^{o b s} \mid x\right) \propto \exp \left\{-\frac{1}{2}\left(y^{o b s}-F(x)\right)^{\mathrm{T}} C_{y}^{-1}\left(y^{o b s}-F(x)\right)\right\} .
$$

Using Bayes' theorem, the posterior distribution of $x$ given the observations $y^{\text {obs }}$ can be written as

$$
\begin{aligned}
\pi\left(x \mid y^{o b s}\right) & =\frac{1}{c} \pi(x) \cdot \pi\left(y^{o b s} \mid x\right) \\
& \propto \exp \left\{-\frac{1}{2}(x-\mu)^{\mathrm{T}} C_{x}^{-1}(x-\mu)-\frac{1}{2}\left(y^{o b s}-F(x)\right)^{\mathrm{T}} C_{y}^{-1}\left(y^{o b s}-F(x)\right)\right\}
\end{aligned}
$$

with $c$ a normalization constant. Markov chain Monte Carlo method (McMC) (Hastings, 1970; Metropolis et al., 1953; Oliver et al., 1997) is suitable for drawing samples from the posterior conditional distribution $\pi\left(x \mid y^{o b s}\right)$. If a sufficiently large chain is generated following the procedure described below, the chain will converge so that its members will be drawn from the posterior conditional distribution. The procedure of McMC is the following (Robert and Casella, 2004)

1. Initialize the first realization $x$.

2. Update $x$ according to the Metropolis-Hastings rule:

- Propose a candidate realization $x^{*}$ conditional on the last realization by drawing from the transition kernel $x^{*} \sim q\left(x^{*} \mid x\right)$.

- Accept the candidate $x^{*}$ with probability $\min \{1, \alpha\}$ and

$$
\alpha=\frac{\pi\left(x^{*} \mid y\right)}{\pi(x \mid y)} \cdot \frac{q\left(x \mid x^{*}\right)}{q\left(x^{*} \mid x\right)}
$$

3. Loop back to the second step.

The two critical points on the McMC method are the selection of the transition kernel $q\left(x^{*} \mid x\right)$ and how to compute the acceptance probability $\alpha$. The first attempt to apply the McMC in hydrogeology is by Oliver et al. (1997) who generated permeability realizations conditioned to variogram and pressure data using a local transition kernel. This local kernel only modifies 
a single cell in the realization of $x$ when making the transition from $x$ to $x^{*}$. Such a small perturbation, specially if the aquifer discretization is large, makes the method quite slow, since after each proposal there is a need to evaluate the state equation (groundwater flow model) and to decide whether the proposal is accepted or not. If transition kernel is global, producing a new realization which changes in every cell of the aquifer, the probability that the candidate is rejected is quite high. An alternative is to use a block kernel in which the proposed realizations differs from the previous one only over a certain region inside the aquifer (Fu and Gómez-Hernández, 2009). The so called blocking McMC gives better results than either the local or global transition kernels. If, in addition, the evaluation of the state of the system, for the purposes of computing the acceptance probability, is made on a coarse scale with the aid of upscaling, and only the high acceptance probability members are submitted to the fine scale evaluation, the convergence rate of the chain will be improved.

As mentioned, the McMC is not an optimization algorithm, it aims at generating multiple independent realizations by sampling from the posterior parameter distribution conditioned on the observations. It is also important to notice that, since the posterior distribution is built from the prior parameter distribution, the realizations generated are consistent with the prior model. We will return later to the issue of whether it is important or not to preserve the prior model structure throughout the inversion process in Section 2.2.6.

The original McMC is computational demanding since each proposed realization is subject to forward simulation which involves solving a partial differential equation over a large domain and a long time period. Furthermore, these proposed realizations are not necessarily accepted, which generally requires that a large number of candidate realizations have to be generated and evaluated. A lot of work has been devoted to increase efficiency of McMC by means of increasing the acceptance rates or reducing the dimensionality of the forward simulation. A "limited-memory" algorithm has been used to accelerate sampling using low-dimensional jump distributions (Kuczera et al., 2010). A two-stage McMC has been proposed to improve the efficiency of McMC (Dostert et al., 2009; Efendiev et al., 2009), in which fine scale simulations are performed only if the proposal at the coarse scale is accepted. Another drawback of McMC is related with the low mixing speed of the chain, in other words, the McMC should sample from the entire posterior distribution, but it takes quite a long chain until this happens (Fu and Gómez-Hernández, 2009; Romary, 2010).

\subsubsection{Real time integration or not?}

The trajectory of inverse modeling up to here shows quite a large evolution from the initial methods. There were two main problems. The first, and most 
important one, was CPU requirements; the second was the need to reformulate the problem from the beginning if new data are collected. Inverse stochastic modeling supposed a big leap in aquifer characterization, but, in essence, it was equivalent to performing inverse modeling seeking a single best estimate, but as many times as realizations were needed. It was necessary to find an alternative capable of generating multiple conditioned realizations of conductivity in a more efficient manner. If this could be achieved, it would be interesting that as new data are collected, as it happens in any monitoring network that measures piezometric heads or concentration values sequentially in time, that they could be incorporated into the inverse model naturally without any modification of the algorithm. The ensemble Kalman filter (EnKF) is the method capable of it (Burgers et al., 1998; Evensen, 1994).

The EnKF is based on the Kalman filter, a data assimilation algorithm for systems in which the relation between model parameters and states is linear (Kalman, 1960). This linearity renders an exact propagation of the covariance with time. However, the equations depicting groundwater and solute transport model are nonlinear (Equations 2.1 and 2.2), which deteriorates the propagation of covariance. As a solution to this problem, the extended Kalman filter was proposed, the nonlinear function is approximated linearly by a Taylorseries expansion and this linearization is used for covariance propagation. The problem with extended Kalman filter is that it also tends to deteriorate as more data assimilated, it does not work well with highly heterogeneous fields, and it is time-consuming when the aquifer is finely discretized (Evensen, 2003).

The EnKF circumvents the problem of covariance propagation in time by working with an ensemble of realizations. The forward problem is solved on each realization, and the ensemble of states is used to compute the covariance explicitly. This is one of the reason that the EnKF is computationally efficient. Another reason resides in that the EnKF is capable of incorporating the observations sequentially in time without the need to store all previous states nor the need to restart groundwater simulation from the very beginning time.

The theory and numerical implementation of the EnKF is described extensively in Evensen $(2003,2007)$. Here we will only recall that the EnKF deals with dynamic systems, for which observed data are obtained as a function of time and used to sequentially update the model. The joint state vector $x_{i}$, for realization $i$, including both the parameters controlling the transfer function and the state variables, is given by:

$$
x_{i}=\left(\begin{array}{l}
A \\
B
\end{array}\right)_{i}=\left(\begin{array}{c}
\left(a_{1}, a_{2}, \ldots, a_{N}\right)^{\mathrm{T}} \\
\left(b_{1}, b_{2}, \ldots, b_{N}\right)^{\mathrm{T}}
\end{array}\right)_{i}
$$

where $A$ is the vector of model parameters such as hydraulic conductivities and porosities, and $B$ is the vector with the state variables such as hydraulic heads and concentrations. The size of the state vector ensemble $\mathrm{x}$ is determined by 
the number of grid cells in which the domain has been discretized $(N)$ and the number of realizations in the ensemble $\left(N_{r}\right)$, i.e., $\mathrm{x}=\left(x_{1}, x_{2}, \ldots, x_{N_{r}}\right)$.

The EnKF consists of two main steps: forecast and update. The forecast step involves the transition of the state vector from time $t-1$ to time $t$, i.e., $\mathbf{x}_{t}=F\left(\mathbf{x}_{t-1}\right)$, where $F(\cdot)$ is the transfer function. Normally this transfer function leaves the model parameters unchanged and forecasts the state variables to the next time step using the groundwater flow model (Equation 2.1). After observation data are collected the state vector is updated by Kalman filtering:

$$
\mathrm{x}_{t}^{u}=\mathrm{x}_{t}^{f}+\mathrm{G}_{t}\left(\mathrm{y}_{t}^{o b s}+\varepsilon-\mathrm{Hx}_{t}^{f}\right)
$$

where $\mathrm{x}_{t}^{u}$ is the joint vector ensemble with the updated states at time $t$ and $\mathrm{x}_{t}^{f}$ is the vector ensemble with the forecasted states; $\mathrm{y}_{t}^{o b s}$ is the observation at time $t ; \varepsilon$ is an observation error characterized by a normal distribution with zero mean and covariance $\mathrm{R}$; $\mathrm{G}_{t}$ is the Kalman gain, derived after the minimization of a posterior error covariance:

$$
\mathrm{G}_{t}=\mathrm{P}_{t}^{f} \mathrm{H}^{\mathrm{T}}\left(\mathrm{HP}_{t}^{f} \mathrm{H}^{\mathrm{T}}+\mathrm{R}\right)^{-1}
$$

it multiplies the residuals between observed and forecasted values to provide an update to the latter; $\mathrm{H}$ is the observation matrix; $\mathrm{P}_{t}^{f}$ is the ensemble covariance matrix of the state $\mathrm{x}_{t}^{f}$ :

$$
\mathrm{P}_{t}^{f} \approx \frac{1}{N_{r}-1}\left(\mathrm{x}_{t}^{f}-\overline{\mathrm{x}}_{t}^{f}\right)\left(\mathrm{x}_{t}^{f}-\overline{\mathrm{x}}_{t}^{f}\right)^{\mathrm{T}}
$$

where $\overline{\mathbf{x}}_{t}^{f}$ is the ensemble mean: $\overline{\mathbf{x}}_{t}^{f} \approx \frac{1}{N_{r}} \sum_{i=1}^{N_{r}} x_{t, i}^{f} ;$ and $x_{t, i}^{f}$ is a realization of the ensemble of state vectors. It is worth noting that we do not have to compute the whole covariance matrix explicitly because we can compute directly $\mathrm{P}_{t}^{f} \mathrm{H}^{\mathrm{T}}$ and $\mathrm{HP}_{t}^{f} \mathrm{H}^{\mathrm{T}}$ taking advantage of the fact that most of the entries of $\mathrm{H}$ are zeroes.

Most inverse methods need to store the previous states when conditioned to new observed data. The forward simulation has to be restarted from the initial time until the new observation data are collected. On the contrary, the EnKF is able to assimilate the real time observation data and stores only the latest state. The EnKF focuses on the present observation data while the previous incorporated observations are not involved any more as soon as they have been assimilated. This is one of the reasons of attractive computational efficiency as we have mentioned. The sequential data assimilation scheme with the EnKF is shown in Figure 2.2. Due to its advantages such as computational efficiency and easiness to combine with any forward model, the EnKF has been widely applied as a data assimilation tool in diverse disciplines such as oceanography, meteorology and hydrology (e.g., Bertino et al., 2003; Chen and Zhang, 2006; 


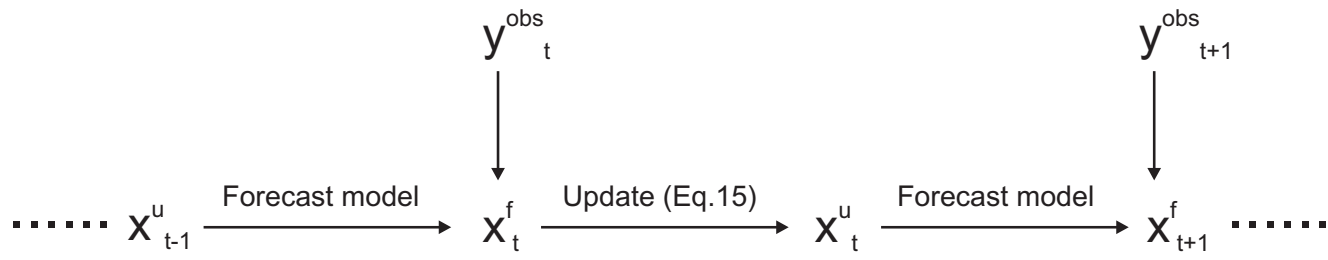

Figure 2.2: Workflow of the EnKF.

Houtekamer and Mitchell, 2001; Moradkhani et al., 2005; Nowak, 2009; Wen and Chen, 2005).

Notice that the EnKF is neither an optimization method nor a sampling one, it is a data assimilation filter based on the minimization of a posterior covariance. For this reason, the EnKF is optimal when parameter and states are linearly related and follow a multiGaussian distribution (Evensen and Leeuwen, 2000). However, in hydrogeology, hydraulic conductivity is likely not to be properly modeled as multiGaussian, and even if it were, the states (heads and concentrations) would never be due to the nonlinear state transfer function. Some work has been done attempting to circumvent the problem of non-Gaussianity, but it will be discussed in its own section later on (Section 2.2.7).

Besides the multiGaussian requirement, other disadvantages related with the EnKF include: (a) underestimation of model variability especially when the ensemble size is small and the parameter is highly heterogeneous; (b) nonphysical and spurious update of state vectors. To address these problems, several covariance regularization strategies are proposed such as covariance inflation, cutoff radius, distance based covariance localization and damping factor scheme (Chen and Oliver, 2010; Devegowda et al., 2010; Hamill et al., 2001; Hendricks Franssen and Kinzelbach, 2008; Houtekamer and Mitchell, 1998).

\subsubsection{Preserve prior structure or not?}

The inverse methods based on optimization attempt to minimize the deviation between predicted states and observation data, disregarding, sometimes, the prior model used to generate the initial guess fields from which to start the optimization. Kitanidis (2007) already stated that "the degree of data reproduction is a poor indicator of the accuracy of estimates". There has been some debate on whether the prior model structure should be preserved through the inverse modeling process, or, on the contrary, the optimization process may allow the final set of realizations to depart (drastically) from the original model as driven by the need to reproduce the observed states. The best, probably 
lies in between, the prior model should be taken into account and should be used as a regularization element, while the new data should allow to introduce some deviations when this is the only way to approximate them. In this respect, Neuman (1973) proposed the multiple-objective algorithm in which the model parameters are constrained not only by minimization of the reproduction error but also by a physical plausibility criterion. A similar strategy is used by Carrera and Neuman (1986a) and Medina and Carrera (1996) in the MLM, in which prior information is combined into the likelihood functions, or by Alcolea et al. (2006) in the PiPM, in which a regularization term is added to the objective function.

There are methods which, by construction, will produce realizations with the prior model structure, such as the McMC, in which the prior model is implicitly built into the definition of the posterior conditional probability distribution from which the chain of realizations are drawn.

There are two other methods that, by construction, will preserve the prior model during the inversion process, thus ensuring that the parameter distributions are physically plausible at the end of the inversion process: the gradual deformation method (GDM) and the probability perturbation method (PrPM).

The GDM method, as initially proposed by Hu (2000), is based in the successive linear combination of pairs of realizations. A single parameter controls this linear combination and its value is computed by a simple optimization procedure so that there is an improvement in the reproduction of the observed state on the resulting realization. In addition, if both realizations are multiGaussian with the same mean and covariance it is easy to show that the resulting realization will also be multiGaussian with the same mean and covariance. This pairwise combination is repeated until an acceptable match to the observed data is attained. This simple, but extremely effective, approach, which only worked for multiGaussian fields was soon extended to work with other random function fields in conjunction with sequential simulation algorithms. In sequential simulation algorithms, each node of the realization is visited sequentially, a random number is drawn and a nodal value is obtained from the local conditional distribution, which has been computed accordingly to the random function model. There are sequential simulation algorithms to generate multiGaussian realizations (e.g., Gómez-Hernández and Journel, 1993), realizations with arbitrary indicator covariance functions (e.g., GómezHernández and Srivastava, 1990) or realizations based on the multiple-point patterns derived from a training image (e.g., Strebelle, 2002). In all cases, it all reduces to mapping a set of independent uniform random numbers into a realization of correlated values using as a transfer function the local conditional probabilities computed according to Bayes' rule, which are the essence of all sequential simulation algorithms. 
The attractiveness of GDM is that each iteration is a simple optimization step, and that it preserves the prior spatial structure. The GDM algorithm can be summarized as follows:

1. Generate two independent Gaussian white noises, $z_{1}$ and $z_{2}$, with zero mean and unit variance. The two noises are combined to form a new Gaussian white noise vector $z$ with zero mean and unit variance according to

$$
z=z_{1} \sin (\rho \pi)+z_{2} \cos (\rho \pi)
$$

where $\rho$ is a deformation parameter ranging from -1 to 1 . If $\rho=0, z$ is the same as $z_{2}$ and if $\rho=1 / 2, z$ is the same as $z_{1}$. Note that more than two noises could be combined to increase the convergence rate (Ying and Gomez-Hernandez, 2000; Le Ravalec-Dupin and Noetinger, 2002).

2. The random vector $z$, is transformed into a uniform white noise vector $u=G^{-1}(z)$, with $G(\cdot)$ being the Gaussian cumulative distribution function, and $u$ is used with a geostatistical sequential simulation algorithm to yield a realization $x, x=S(z)$.

3. Run the forward model $F(\cdot)$ (e.g., Equation 2.1) on the generated property realization to obtain the simulated model responses, such as flow rates and hydraulic heads.

4. An objective function measuring the mismatch between the simulated model response and the observed data is built as

$$
J(\rho)=\sum_{i=1}^{n} \omega_{i}\left(F(x(\rho))_{i}-F(x(\rho))_{i}^{o b s}\right)^{2} .
$$

where $\omega_{i}$ is a weight and $n$ is the number of observed data.

5. Minimize the objective function to obtain the optimal $\rho$, and update the random vector $z$.

6. The updated random vector $z$ will replace the previous $z_{1}$ and combine with a newly generated vector $z_{2}$ to construct a new random vector. Then loop back to the second step until all the observed data are matched up to some tolerance.

The drawback of the GDM is related to the convergence rate. Le RavalecDupin and Noetinger (2002) found that the convergence rate is strongly influenced by the number of realizations which are combined in each iteration. Caers (2003) proposed an efficient gradual deformation algorithm by coupling the traditional GDM, multiple-point geostatistics and a fast streamline-based 
history matching method with the aim to reduce CPU demand for parameter identification in highly heterogeneous reservoir. Another criticism to GDM has been whether it generates realizations spanning the entire space of variability coherent with the observed data; in this respect, Liu and Oliver (2004) assessed the performance of the GDM through a one-dimensional experiment; after comparison of the GDM with other inverse methods, such as the McMC, they concluded that the GDM is able to produce reasonable distributions of permeability and porosity in their model.

Another method that attempts to preserve the prior structural model is the probability perturbation method (Caers, 2003). The probability perturbation is also based on the sequential simulation algorithm, therefore, it will preserve the random function model that is implicit to the algorithm used. Given a fixed random path to visit the nodes of the aquifer, a fixed set of random numbers, and a fixed set of conditional probability distributions, the PrPM will freeze the random numbers and perturb the conditional probability distributions in order to achieve the match to the observed data. Recall that the GDM freezes the probability distributions and modifies the random numbers. The perturbation of the conditional probabilities is performed by means of a single parameter $r_{D}$ that is subject to optimization. This parameter can be interpreted as the degree of perturbation needed to apply to the seed realization in order to match the state data, if $r_{D}$ is close to zero, the actual realization gives a good reproduction of the state data, there is no need to change anything, if $r_{D}$ is close to one, the current realization is far from matching the observation data and there is a need to generate another realization independent of the previous one, any value in between would generate a realization that represents a transition between these two independent realizations. Caers (2007) compared the performances of the GDM an the PrPM in several simple examples from such aspects as preservation of prior structure and accuracy of posterior sampler.

The PrPM method has been extended to allow different degree of perturbation in different geological zones of the aquifer, that is, $r_{D}$ is allowed to vary piecewise within the aquifer according to pre-defined zones (Hoffman and Caers, 2005). The PrPM was initially applied to categorical variables, although later it was extended to continuous ones $(\mathrm{Hu}, 2008)$. The PrPM has been mostly used in petroleum engineering although recently it has been applied in groundwater modeling, e.g., combined with a multiple-point geostatistical method to locate high permeability zones in an aquifer (Ronayne et al., 2008).

Before Caers proposed the PrPM, the idea of perturbing probabilities had already been proposed by Capilla et al. (1999) although in a slightly different context. They used the concept of the SCM method, but instead of working with the conductivity values directly, they transformed these conductivity 
values onto cumulative probabilities using the local conditioning probability distributions obtained by kriging (the probability field of Froidevaux (1993)). The type of kriging used could be indicator kriging and could incorporate soft conditioning data, and therefore, the spatial structure associated to such a type of kriging would be preserved through the optimization process. Once the probabilities had been computed, the SCM method would be applied to seek the best spatial distribution of probabilities that when backtransformed onto conductivities would result in the best match to the observed state data. Later, to improve its efficiency, the optimization step of the probability fields was combined with the GDM by Capilla and Llopis-Albert (2009).

The problem still is what if the prior model is not correct. What if the prior model implies an isotropic spatial correlation, but, in reality conductivities are highly anisotropic with channels of high permeability and quasi impermeable barriers? Some studies have analyzed the impact of a wrong a priori model choice, for instance, Freni and Mannina (2010) analyzed the impact of different a priori hypotheses and found that improper assumptions could lead to very poor parameter estimations; Li et al. (2011c) assessed the performance of normal-score EnKF (NS-EnKF) in non-multiGaussian media and they argued that, if the monitoring net was designed properly, the localized NS-EnKF was able to identify the channel structure even when an erroneous prior random function model was used. A possible solution to account for the prior model uncertainty is to use multiple prior models as done by Suzuki and Caers (2008) although at a very large computational demand.

\subsubsection{MultiGaussian or not?}

Ever since the publication of the seminal paper on stochastic hydrogeology by Freeze (1975), hydraulic conductivity has been assumed to follow a univariate lognormal distribution. This assumption was based on experimental data, and it was later corroborated by Hoeksema and Kitanidis (1985) who analyzed the histogram and covariance of hydraulic conductivity data from 31 regional aquifers to conclude that, indeed, hydraulic conductivity is best modeled by a logGaussian histogram. However, there are still many cases, such as aquifers in fluvial deposits, in which several highly contrasting facies coexist and in which conductivities are better characterized by a multimodal distribution. But, whether the histogram of the conductivities is normal is not the most important issue, after all, it is always possible to apply a normal-score transformation to the data so that the transformed data follows a Gaussian histogram; the important issue is whether the best model to characterize the spatial continuity of hydraulic conductivity is the multiGaussian or not. Applying a normal-score transform to the data will render them univariate normal, but it does not imply that the higher-order models (the ones controlling 
the continuity of the extreme values, or the curvilinear arrangements of some conductivity values) should follow a multiGaussian model.

The nonGaussian models have been explored for some time now (e.g., Gómez-Hernández and Wen, 1998; Journel and Deutsch, 1993; Kerrou et al., 2008; Rubin and Journel, 1991; Woodbury and Ulrych, 1993; Zinn and Harvey, 2003), and the dangers of using a multiGaussian model in aquifers with high continuity of extreme values were already exposed by Journel and Deutsch (1993) and Gómez-Hernández and Wen (1998).

Of all the methods discussed, all of those which are based on the minimization of the sum of square deviations have a tendency to generate multiGaussian realizations, even if the seed realizations prior to the start of the inverse procedure are non-Gaussian. Basically, all methods that use only moments up to the order two (covariance) in their formulation will behave in this way as a consequence that the multiGaussian distribution is the only one fully characterized by a mean value and a two-point covariance function. This is the case of the GA, the SCM, the PiPM, or the MLM. Even the EnKF, which only uses the ensemble derived covariance to update the realizations after each data collection stage, will end up with realizations with a multiGaussian flavor even if the initial ensemble is not.

Some of the methods discussed can handle non multiGaussian patterns of variability, such as the McMC, the GDM or the PrPM. It is apparent that those methods that can benefit from techniques such as multiple point geostatistical simulation, which can generate realizations of conductivity with realistic patterns according to a training image (Mariethoz, 2009), are the most promising. There have been some attempts to modify the EnKF to handle non multiGaussian ensembles, by applying transformations to parameter and state variables that either makes them Gaussian (Zhou et al., 2011a; Schöniger et al., 2011) or linearize their relationship (Schöniger, 2010).

\subsubsection{Trajectory of inverse approaches to date}

Table 2.1 summarizes the inverse methods discussed so far. As time has passed inverse models have, sequentially, gotten away from the linearization of the state equation, become stochastic, attempted to preserve the prior structure (or at least introduce some controls which will give the prior structure information some weight during the inverse modeling process), and become capable of handling non-multiGaussian realizations. In our opinion the best inverse model should be the one that is stochastic, is capable to deal with multiple sources of state data governed by a complex state equation, is not limited to multiGaussian realizations, can weight in prior information, and can generate multiple realizations in an efficient manner. 


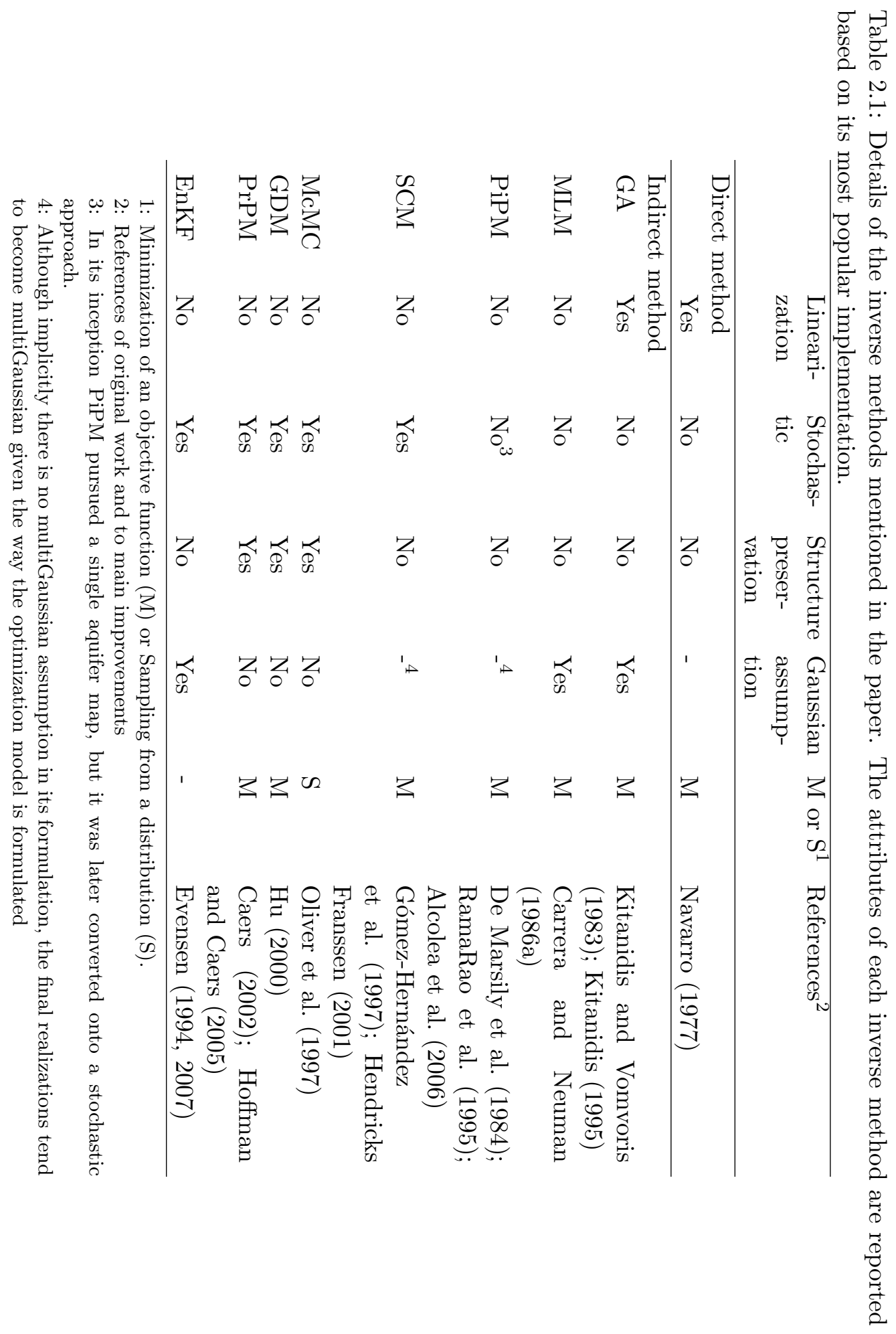




\subsection{Recent trend of inverse methods}

The methods discussed so far have already been thoroughly tested and their advantages and pitfalls are well known. In the last few years, new issues have been brought into the inverse model formulation that we would like to mention next.

\subsubsection{Integrating multi-sources information}

Direct measurements of parameters of interest (usually known as "hard data") represent the first constraint that the model must meet. These data are easily handled by standard geostatistic methods (Deutsch and Journel, 1998). Then, there are soft data, that is, indirect measurements of the parameters. Recent developments in physical and geophysical techniques provide indirect measurements that are non-linearly related to the parameter of interest and that should be used also to constrain our aquifer model. Examples of these techniques are ground-penetrating radar (Dafflon et al., 2009; Kowalsky et al., 2004), time-lapse electrical resistivity (Irving and Singha, 2010), 4D-seismic (Le Ravalec-Dupin, 2010), spatial altimetry (Getirana, 2010), and remote sensing (Brunner et al., 2007; Hendricks Franssen et al., 2008). It is worth noting that certain techniques (e.g., remote sensing) are able to provide information over a large area rather than at quasi-point scale. Besides the hard and soft (geo)physical measurements, state data other than hydraulic heads or flow rates should be used to infer aquifer parameters, for instance peak concentration arrival times (Bellin and Rubin, 2004) or groundwater ages (Sanford, 2010).

Two other types of data have been used to identify aquifer structure, i.e., water table fluctuations due to tides and connectivity data. Tidal induced water table fluctuations carry information about the aquifer properties in coastal aquifers. Head fluctuations have been used to identify possible preferential flow paths between the sea and the coastal aquifer (Alcolea et al., 2007; Park et al., 2011; Slooten et al., 2010). The other rarely applied constraint is connectivity, which is found to play a critical role in transport modeling. In a synthetic example, Zinn and Harvey (2003) demonstrated how the same conductivity values when rearranged in space to induce different connectivity patterns have very different flow and transport behavior. Some indicators has been proposed to measure the connectivity (e.g., Knudby and Carrera, 2005, 2006; Le Goc et al., 2010), and some attempts to include connectivity information in inverse modeling have been carried out (Alcolea and Renard, 2010; Renard and Caers, 2008). 


\subsubsection{Combining high-order moments}

To include curvilinear features in the spatial distribution of the hydraulic conductivities amounts to go beyond the two-point covariance (a second order moment) and to account for high-order moments. A possible approach is with multiple-point geostatistics (Guardiano and Srivastava, 1993; Strebelle, 2002). We have already discussed how the GDM and the PrPM take advantage of the sequential simulation methods based on multiple-point geostatistics to generate inverse conditional realizations following the patterns extracted from a training image (such as the one in Figure 2.3). An alternative avenue is the use of spatial cumulants (Mustapha and Dimitrakopoulos, 2010). Other examples include that Alcolea and Renard (2010) proposed a blocking moving window algorithm taking advantage of multiple point simulations, Mariethoz et al. (2010) has proposed an iterative resampling algorithm, and Zhou et al. (2011b) presented a pattern search based inverse method.

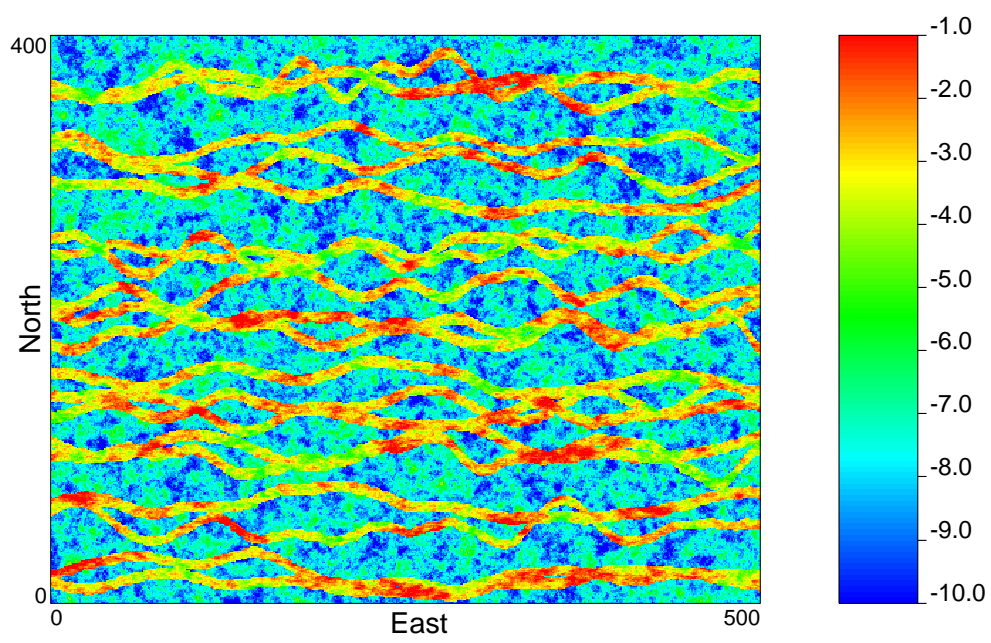

Figure 2.3: Conceptual model of a fluvial deposited aquifer used as a training image by the multiple point based simulation algorithms.

\subsubsection{Multiscale problem}

There are a couple of reasons why it is interesting to use a multiscale approach to deal with the inverse problem. On one hand, if the aquifer is very heterogeneous and the discretization is too fine, it may be computationally impossible to handle a stochastic inverse problem. On the other hand, data may be available at different support scales. Some authors have combined upscaling and 
inverse modeling to address the scale problem (e.g., Fu and Gómez-Hernández, 2009; Fu et al., 2010; Li et al., 2011d; Li and Ren, 2010; Scheidt et al., 2011).

\subsection{Conclusions}

We have given an overview of the trajectory of inverse methods in hydrogeology, i.e., how the algorithms have evolved during the last decades to solve the inverse problem, from direct solutions to indirect methods, from linearization to nonlinearization of the transfer function, and from single estimate to stochastic Monte Carlo simulation. Furthermore, we consider a few issues involved in solving the inverse problem, e.g., whether the multiGaussian assumption is appropriate and whether the prior structure should serve as a constraint. Not all aspects of inverse modeling have been touched here, the reader is referred to other works for a more general introduction to the inverse problem (e.g., Oliver et al., 2008; Sun, 1994). 


\section{Bibliography}

Alcolea, A., Carrera, J., Medina, A., 2006. Pilot points method incorporating prior information for solving the groundwater flow inverse problem. Advances in Water Resources 29 (11), 1678-1689.

Alcolea, A., Carrera, J., Medina, A., 2008. Regularized pilot points method for reproducing the effect of small scale variability: Application to simulations of contaminant transport. Journal of Hydrology 355 (1-4), 76-90.

Alcolea, A., Castro, E., Barbieri, M., Carrera, J., Bea, S., 2007. Inverse modeling of coastal aquifers using tidal response and hydraulic tests. Ground Water 45 (6), 711-722.

Alcolea, A., Renard, P., 2010. Blocking Moving Window algorithm: Conditioning multiple-point simulations to hydrogeological data. Water Resources Research 46 (8), W08511.

Bear, J., 1972. Dynamics of fluids in porous media. American Elsevier Pub. Co., New York, 764pp.

Bellin, A., Rubin, Y., 2004. On the use of peak concentration arrival times for the inference of hydrogeological parameters. Water Resources Research 40 (7), W07401.

Bertino, L., Evensen, G., Wackernagel, H., 2003. Sequential data assimilation techniques in oceanography. International Statistical Review 71 (2), 223241.

Brouwer, G. K., Fokker, P. A., Wilschut, F., Zijl, W., 2008. A direct inverse model to determine permeability fields from pressure and flow rate measurements. Mathematical Geosciences 40 (8), 907-920.

Brunner, P., Hendricks Franssen, H. J., Kgotlhang, L., Bauer-Gottwein, P., Kinzelbach, W., 2007. How can remote sensing contribute in groundwater modeling? Hydrogeology Journal 15 (1), 5-18.

Burgers, G., Jan van Leeuwen, P., Evensen, G., 1998. Analysis scheme in the ensemble Kalman filter. Monthly Weather Review 126 (6), 1719-1724. 
Caers, J., 2002. Geostatistical history matching under training-image based geological model constraints. SPE Annual Technical Conference and Exhibition, San Antonio, Texas, SPE 77429.

Caers, J., 2003. Efficient gradual deformation using a streamline-based proxy method. Journal of Petroleum Science and Engineering 39 (1-2), 57-83.

Caers, J., 2007. Comparing the gradual deformation with the probability perturbation method for solving inverse problems. Mathematical Geology 39 (1), 27-52.

Capilla, J., Llopis-Albert, C., 2009. Gradual conditioning of non-Gaussian transmissivity fields to flow and mass transport data: 1 . Theory. Journal of Hydrology 371 (1-4), 66-74.

Capilla, J. E., Gómez-Hernández, J. J., Sahuquillo, A., 1997. Stochastic simulation of transmissivity fields conditional to both transmissivity and piezometric data 2. Demonstration on a synthetic aquifer. Journal of Hydrology 203 (1-4), 175-188.

Capilla, J. E., Gómez-Hernández, J. J., Sahuquillo, A., 1998. Stochastic simulation of transmissivity fields conditional to both transmissivity and piezometric head data-3. Application to the Culebra Formation at the Waste Isolation Pilot Plan (WIPP), New Mexico, USA. Journal of Hydrology 207 (34), 254-269.

Capilla, J. E., Rodrigo, J., Gómez-Hernández, J. J., 1999. Simulation of nonGaussian transmissivity fields honoring piezometric data and integrating soft and secondary information. Mathematical Geology 31 (7), 907-927.

Carrera, J., 1987. State of the art of the inverse problem applied to the flow and solute transport equations. In: Custodio, E., Gurgui, A., Ferreira, J. L. (Eds.), Analytical and Numerical Groundwater Flow and Quality Modelling. Series C, Mathematical and physical sciences. Vol. 224. D. Reidel, Norwell, MA, pp. 549-583.

Carrera, J., Alcolea, A., Medina, A., Hidalgo, J., Slooten, L. J., 2005. Inverse problem in hydrogeology. Hydrogeology Journal 13 (1), 206-222.

Carrera, J., Neuman, S. P., 1986a. Estimation of aquifer parameters under transient and steady state conditions: 1 . Maximum likelihood method incorporating prior information. Water Resources Research 22 (2), 199-210.

Carrera, J., Neuman, S. P., 1986b. Estimation of aquifer parameters under transient and steady state conditions: 2. uniqueness, stability, and solution algorithms. Water Resources Research 22 (2), 211-227. 
Chen, Y., Oliver, D. S., 2010. Cross-covariances and localization for EnKF in multiphase flow data assimilation. Computational Geosciences 14 (4), $579-601$.

Chen, Y., Zhang, D., 2006. Data assimilation for transient flow in geologic formations via ensemble Kalman filter. Advances in Water Resources 29 (8), $1107-1122$.

Cooley, R. L., Hill, M. C., 2000. Comment on RamaRao et al.[1995] and LaVenue et al.[1995]. Water Resources Research 36 (9), 2795-2797.

Dafflon, B., Irving, J., Holliger, K., 2009. Use of high-resolution geophysical data to characterize heterogeneous aquifers: Influence of data integration method on hydrological predictions. Water Resources Research 45 (9), W09407.

Dagan, G., 1985. Stochasti modeling of groundwater flow by unconditional and conditional probabilities: The inverse problem. Water Resources Research $21(1), 65-72$.

Dagan, G., 1986. Statistical theory of groundwater flow and transport: Pore to laboratory, laboratory to formation, and formation to regional scale. Water Resources Research 22 (9), 120S-134S.

De Marsily, G., Delhomme, J. P., Coudrain-Ribstein, A., Lavenue, A. M., 2000. Four decades of inverse problems in hydrogeology. In: Zhang, D., Winter, C. L. (Eds.), Theory, modeling, and field investigation in hydrogeology: a special volume in honor of Shlomo P. Neumans 60th birthday. Geological Society of America Special Paper 348, Boulder, Colorado, pp. $1-17$.

De Marsily, G., Lavedau, G., Boucher, M., Fasanino, G., 1984. Interpretation of interference tests in a well field using geostatistical techniques to fit the permeability distribution in a reservoir model. In: Verly, G., David, M., Journel, A. G., Marechal, A. (Eds.), Geostatistics for natural resources characterization. D. Reidel, Hingham, Mass., pp. 831-849.

Deutsch, C. V., Journel, A. G., 1998. GSLIB, Geostatistical Software Library and User's Guide, 2nd Edition. Oxford University Press, New York, 384pp.

Devegowda, D., Arroyo-Negrete, E., Datta-Gupta, A., 2010. Flow relevant covariance localization during dynamic data assimilation using EnKF. Advances in Water Resources 33, 129-145. 
Dostert, P., Efendiev, Y., Mohanty, B., 2009. Efficient uncertainty quantification techniques in inverse problems for Richards' equation using coarse-scale simulation models. Advances in Water Resources 32 (3), 329-339.

Efendiev, Y., Datta-Gupta, A., Ma, X., Mallick, B., 2009. Efficient sampling techniques for uncertainty quantification in history matching using nonlinear error models and ensemble level upscaling techniques. Water Resources Research 45 (11), W11414.

Emsellem, Y., De Marsily, G., 1971. An automatic solution for the inverse problem. Water Resources Research 7 (5), 1264-1283.

Evensen, G., 1994. Sequential data assimilation with a nonlinear quasigeostrophic model using Monte Carlo methods to forecast error statistics. Journal of Geophysical Research 99 (C5), 10143-10162.

Evensen, G., 2003. The Ensemble Kalman Filter: Theoretical formulation and practical implementation. Ocean dynamics 53 (4), 343-367.

Evensen, G., 2007. Data assimilation: The ensemble Kalman filter. Springer Verlag, 279pp.

Evensen, G., Leeuwen, P. J. V., 2000. An ensemble kalman smoother for nonlinear dynamics. Monthly Weather Review 128, 1852-1867.

Ferraresi, M., Todini, E., Vignoli, R., 1996. A solution to the inverse problem in groundwater hydrology based on Kalman filtering. Journal of Hydrology 175 (1-4), 567-581.

Freeze, R. A., 1975. A stochastic-conceptual analysis of one-dimensional groundwater flow in nonuniform homogeneous media. Water Resources Research 11 (3), 725-741.

Freni, G., Mannina, G., 2010. Bayesian approach for uncertainty quantification in water quality modelling: the influence of prior distribution. Journal of Hydrology, doi: 10.1016/j.jhydrol.2010.07.043.

Froidevaux, R., 1993. Probability field simulation. In: Soares, A. (Ed.), Geostatistics Tróia '92, volume 1. Kluwer, pp. 73-84.

Fu, J., Gómez-Hernández, J. J., 2009. A blocking Markov chain Monte Carlo method for inverse stochastic hydrogeological modeling. Mathematical Geosciences 41 (2), 105-128.

Fu, J., Tchelepi, H. A., Caers, J., 2010. A multiscale adjoint method to compute sensitivity coefficients for flow in heterogeneous porous media. Advances in Water Resources, doi: 10.1016/j.advwatres.2010.04.005. 
Getirana, A. C. V., 2010. Integrating spatial altimetry data into the automatic calibration of hydrological models. Journal of Hydrology 387 (3-4), 244-255.

Gómez-Hernández, J. J., Hendricks Franssen, H. J. W. M., Sahuquillo, A., 2003. Stochastic conditional inverse modeling of subsurface mass transport: A brief review and the self-calibrating method. Stochastic Environmental Research and Risk Assessment 17 (5), 319-328.

Gómez-Hernández, J. J., Journel, A. G., 1993. Joint sequential simulation of Multi-Gaussian fields. In: Soares, A. (Ed.), Geostatistics Tróia '92. Vol. 1. Kluwer Academic Publishers, Dordrecht, pp. 85-94.

Gómez-Hernández, J. J., Sahuquillo, A., Capilla, J. E., 1997. Stochastic simulation of transmissivity fields conditional to both transmissivity and piezometric data-I. Theory. Journal of Hydrology 203 (1-4), 162-174.

Gómez-Hernández, J. J., Srivastava, R. M., 1990. ISIM3D: an ANSI-C three dimensional multiple indicator conditional simulation program. Computer and Geosciences 16 (4), 395-440.

Gómez-Hernández, J. J., Wen, X., 1994. Probabilistic assessment of travel times in groundwater modeling. J. of Stochastic Hydrology and Hydraulics 8 (1), 19-56.

Gómez-Hernández, J. J., Wen, X. H., 1998. To be or not to be multi-Gaussian? A reflection on stochastic hydrogeology. Advances in Water Resources 21 (1), $47-61$.

Guardiano, F., Srivastava, R., 1993. Multivariate geostatistics: beyond bivariate moments. In: Soares, A. (Ed.), Geostatistics-Troia. Kluwer Academic Publ, Dordrecht, pp. 133-144.

Hamill, T. M., Whitaker, J. S., Snyder, C., 2001. Distance-dependent filtering of background error covariance estimates in an ensemble Kalman filter. Monthly Weather Review 129 (11), 2776-2790.

Hastings, W. K., 1970. Monte Carlo sampling methods using Markov chains and their applications. Biometrika 57 (1), 97-109.

Hendricks Franssen, H.-J., 2001. Inverse stochasstic modelling of groundwater flow and mass transport. Ph.D. thesis, Technical University of Valencia, Spain. 363pp.

Hendricks Franssen, H. J., Alcolea, A., Riva, M., Bakr, M., van der Wiel, N., Stauffer, F., Guadagnini, A., 2009. A comparison of seven methods for the inverse modelling of groundwater flow. Application to the characterisation of well catchments. Advances in Water Resources 32 (6), 851-872. 
Hendricks Franssen, H. J., Brunner, P., Makobo, P., Kinzelbach, W., 2008. Equally likely inverse solutions to a groundwater flow problem including pattern information from remote sensing images. Water Resources Research 44, W01419.

Hendricks Franssen, H. J., Gómez-Hernández, J. J., Sahuquillo, A., 2003. Coupled inverse modelling of groundwater flow and mass transport and the worth of concentration data. Journal of Hydrology 281 (4), 281-295.

Hendricks Franssen, H. J., Kinzelbach, W., 2008. Real-time groundwater flow modeling with the Ensemble Kalman Filter: Joint estimation for states and parameters and the filter inbreeding problem. Water Resources Research 44, W09408.

Hoeksema, R. J., Kitanidis, P. K., 1984. An application of the geostatistical approach to the inverse problem in two-dimensional groundwater modeling. Water Resources Research 20 (7), 1003-1020.

Hoeksema, R. J., Kitanidis, P. K., 1985. Analysis of the spatial structure of properties of selected aquifers. Water Resources Research 21 (4), 563-572.

Hoffman, B. T., Caers, J., 2005. Regional probability perturbations for history matching. Journal of Petroleum Science and Engineering 46 (1-2), 53-71.

Houtekamer, P. L., Mitchell, H. L., 1998. Data assimilation using an ensemble Kalman filter technique. Monthly Weather Review 126 (3), 796-811.

Houtekamer, P. L., Mitchell, H. L., 2001. A sequential ensemble Kalman filter for atmospheric data assimilation. Monthly Weather Review 129 (1), 123137.

Hu, L. Y., 2000. Gradual deformation and iterative calibration of Gaussianrelated stochastic models. Mathematical Geology 32 (1), 87-108.

Hu, L. Y., 2008. Extended Probability Perturbation Method for Calibrating Stochastic Reservoir Models. Mathematical Geosciences 40 (8), 875-885.

Huang, H., Hu, B. X., Wen, X. H., Shirley, C., 2004. Stochastic inverse mapping of hydraulic conductivity and sorption partitioning coefficient fields conditioning on nonreactive and reactive tracer test data. Water Resources Research 40 (1), W01506.

Irving, J., Singha, K., 2010. Stochastic inversion of tracer test and electrical geophysical data to estimate hydraulic conductivities. Water Resources Research 46 (11), W11514. 
Journel, A. G., 1974. Geostatistics for conditional simulation of ore bodies. Economic Geology 69 (5), 673-687.

Journel, A. G., Deutsch, C. V., 1993. Entropy and spatial disorder. Mathematical Geology 25 (3), 329-355.

Kalman, R. E., 1960. A new approach to linear filtering and prediction problems. Journal of Basic Engineering 82, 35-45.

Kashyap, R., 1982. Optimal choice of AR and MA parts in autoregressive moving average models. IEEE Transactions on Pattern Analysis and Machine Intelligence PAMI-4 (2), 99-104.

Kerrou, J., Renard, P., Hendricks Franssen, H. J., Lunati, I., 2008. Issues in characterizing heterogeneity and connectivity in non-multiGaussian media. Advances in Water Resources 31 (1), 147-159.

Kitanidis, P., 1996. On the geostatistical approach to the inverse problem. Advances in Water Resources 19 (6), 333-342.

Kitanidis, P. K., 1995. Quasi-linear geostatistical theory for inversing. Water Resources Research 31 (10), 2411-2419.

Kitanidis, P. K., 2007. On stochastic inverse modeling. In: Hyndman, D. W. Day-Lewis, F. D., Singha, K. (Eds.), Subsurface hydrology: Data integration for properties and processes. American Geophysical Union, Washington, DC, pp. 19-30.

Kitanidis, P. K., Vomvoris, E. G., 1983. A geostatistical approach to the inverse problem in groundwater modeling (steady state) and one-dimensional simulations. Water Resources Research 19 (3), 677-690.

Kleinecke, D., 1971. Use of linear programing for estimating geohydrologic parameters of groundwater basins. Water Resources Research 7 (2), 367374 .

Knudby, C., Carrera, J., 2005. On the relationship between indicators of geostatistical, flow and transport connectivity. Advances in Water Resources 28 (4), 405-421.

Knudby, C., Carrera, J., 2006. On the use of apparent hydraulic diffusivity as an indicator of connectivity. Journal of Hydrology 329 (3-4), 377-389.

Kowalsky, M. B., Finsterle, S., Rubin, Y., 2004. Estimating flow parameter distributions using ground-penetrating radar and hydrological measurements during transient flow in the vadose zone. Advances in Water Resources 27 (6), 583-599. 
Kuczera, G., Kavetski, D., Renard, B., Thyer, M., 2010. A limited-memory acceleration strategy for MCMC sampling in hierarchical Bayesian calibration of hydrological models. Water Resources Research 46, W07602.

LaVenue, A. M., RamaRao, B. S., De Marsily, G., Marietta, M. G., 1995. Pilot point methodology for automated calibration of an ensemble of conditionally simulated transmissivity fields 2. Application. Water Resources Research $31(3), 495-516$.

Lavenue, M., De Marsily, G., 2001. Three-dimensional interference test interpretation in a fractured aquifer using the pilot point inverse method. Water Resources Research 37 (11), 2659-2675.

Le Goc, R., de Dreuzy, J.-R., Davy, P., 2010. Statistical characteristics of flow as indicators of channeling in heterogeneous porous and fractured media. Advances in Water Resources 33 (3), 257-269.

Le Ravalec-Dupin, M., 2010. Pilot block method methodology to calibrate stochastic permeability fields to dynamic data. Mathematical Geosciences $42(2), 165-185$.

Le Ravalec-Dupin, M., Noetinger, B., 2002. Optimization with the gradual deformation method. Mathematical Geology 34 (2), 125-142.

Li, L., Zhou, H., Gómez-Hernández, J. J., 2011a. A comparative study of three-dimensional hydraulic conductivity upscaling at the macro-dispersion experiment (MADE) site, Columbus Air Force Base, Mississippi (USA). Journal of Hydrology 404 (3-4), 278-293.

Li, L., Zhou, H., Gómez-Hernández, J. J., 2011b. Transport upscaling using multi-rate mass transfer in three-dimensional highly heterogeneous porous media. Advances in Water Resources 34 (4), 478-489.

Li, L., Zhou, H., Hendricks Franssen, H. J., Gómez-Hernández, J. J., 2011c. Groundwater flow inverse modeling in non-MultiGaussian media: performance assessment of the normal-score Ensemble Kalman Filter. Hydrology and Earth System Sciences Discussions 8 (4), 6749-6788.

Li, L., Zhou, H., Hendricks Franssen, H.-J., Gómez-Hernández, J. J., 2011d. Modeling transient groundwater flow by coupling ensemble Kalman filtering and upscaling. Water Resources Research, under review.

Li, N., Ren, L., 2010. Application and assessment of a multiscale data integration method to saturated hydraulic conductivity in soil. Water Resources Research 46, W09510. 
Liu, N., Oliver, D. S., 2004. Experimental assessment of gradual deformation method. Mathematical Geology 36 (1), 65-77.

Mariethoz, G., 2009. Geological stochastic imaging for aquifer characterization. Ph.D. thesis, Universite de Neuchatel.

Mariethoz, G., Renard, P., Caers, J., 2010. Bayesian inverse problem and optimization with iterative spatial resampling. Water Resources Research 46 (11), W11530.

McLaughlin, D., Townley, L. R., 1996. A reassessment of the groundwater inverse problem. Water Resources Research 32 (5), 1131-1161.

Medina, A., Carrera, J., 1996. Coupled estimation of flow and solute transport parameters. Water Resources Research 32 (10), 3063-3076.

Medina, A., Carrera, J., 2003. Geostatistical inversion of coupled problems: dealing with computaional burden and different types of data. Journal of Hydrology 281 (4), 251-264.

Metropolis, N., Rosenbluth, A. W., Rosenbluth, M. N., Teller, A. H., Teller, E., 1953. Equation of state calculations by fast computing machines. The journal of chemical physics 21 (6), 1087-92.

Moradkhani, H., Sorooshian, S., Gupta, H. V., Houser, P. R., 2005. Dual state-parameter estimation of hydrological models using ensemble Kalman filter. Advances in Water Resources 28 (2), 135-147.

Mustapha, H., Dimitrakopoulos, R., 2010. A new approach for geological pattern recognition using high-order spatial cumulants. Computers \& Geosciences 36 (3), 313-334.

Navarro, A., 1977. A modified optimization method of estimating aquifer parameters. Water Resources Research 13 (6), 935-939.

Neuman, S. P., 1973. Calibration of distributed parameter groundwater flow models viewed as a multiple-objective decision process under uncertainty. Water Resources Research 9 (4), 1006-1021.

Neuman, S. P., 2006. Blueprint for perturbative solution of flow and transport in strongly heterogeneous composite media using fractal and variational multiscale decomposition. Water Resources Research 42, W06D04.

Nowak, W., 2009. Best unbiased ensemble linearization and the quasi-linear Kalman ensemble generator. Water Resour Res 45, W04431. 
Oliver, D. S., Chen, Y., 2010. Recent progress on reservoir history matching: a review. Computational Geosciences 15 (1), 185-221.

Oliver, D. S., Cunha, L. B., Reynolds, A. C., 1997. Markov chain Monte Carlo methods for conditioning a permeability field to pressure data. Mathematical Geology 29 (1), 61-91.

Oliver, D. S., Reynolds, A. C., Liu, N., 2008. Inverse theory for petroleum reservoir characterization and history matching. Cambridge University Press.

Park, E., Kim, K.-Y., Ding, G., Kim, K., Han, W. S., Kim, Y., Kim, N., 2011. A delineation of regional hydraulic conductivity based on water table fluctuation. Journal of Hydrology, doi: 10.1016/j.jhydrol.2011.01.002.

Poeter, E. P., Hill, M. C., 1997. Inverse models: A necessary next step in ground-water modeling. Ground water 35 (2), 250-260.

Ponzini, G., Lozej, A., 1982. Identification of aquifer transmissivities: The comparison model method. Water Resources Research 18 (3), 597-622.

RamaRao, B., LaVenue, A., De Marsily, G., Marietta, M., 1995. Pilot point methodology for automated calibration of an ensemble of conditionally simulated transmissivity fields 1 . Theory and computational experiments. Water Resources Research 31 (3), 475-493.

RamaRao, B. S., LaVenue, A. M., de Marsily, G., Marietta, M. G., 2000. Reply to Comment on RamaRao et al.[1995] and LaVenue et al.[1995]. Water resources research 36 (9), 2799-2803.

Renard, P., Caers, J., 2008. Conditioning facies simulations with connectivity data. In: Ortiz, J. M., Emery, X. (Eds.), Proceedings of the eighth International Geostatistical Congress. Santiago, Chile, pp. 597-606.

Renard, P., Marsily, G. D., 1997. Calculating equivalent permeability: A review. Advances in Water Resources 20 (5-6), 253-278.

Robert, C. P., Casella, G., 2004. Monte Carlo statistical methods. Springer Verlag.

Romary, T., 2010. History matching of approximated lithofacies models under uncertainty. Computational Geosciences 14 (2), 343-355.

Ronayne, M. J., Gorelick, S. M., Caers, J., 2008. Identifying discrete geologic structures that produce anomalous hydraulic response: An inverse modeling approach. Water Resources Research 44 (8), W08426. 
Rubin, Y., Chen, X., Murakami, H., Hahn, M., 2010. A Bayesian approach for inverse modeling, data assimilation, and conditional simulation of spatial random fields. Water Resources Research 46, W10523.

Rubin, Y., Dagan, G., 1987. Stochastic identification of transmissivity and effective recharge in steady groundwater flow 1. theory. Water Resources Research 23 (7), 1185-1192.

Rubin, Y., Journel, A. G., 1991. Simulation of non-Gaussian space random functions for modeling transport in groundwater. Water Resources Research 27 (7), 1711-1721.

Sagar, B., Yakowitz, S., Duckstein, L., 1975. A direct method for the identification of the parameters of dynamic nonhomogeneous aquifers. Water Resources Research 11 (4), 563-570.

Sahuquillo, A., Capilla, J. E., Gómez-Hernández, J. J., 1992. Conditional simulation of transmissivity fields honouring piezometric data. In: Blain, W. R., Cabrera, E. (Eds.), Hydraulic Engineering Software IV, Fluid Flow Modeling. Kluwer Academic Publishers.

Sanchez-Vila, X., Guadagnini, A., Carrera, J., 2006. Representative hydraulic conductivities in saturated groundwater flow. Reviews of Geophysics 44 (3), RG3002.

Sanford, W., 2010. Calibration of models using groundwater age. Hydrogeology Journal, doi: 10.1007/s10040-010-0637-6.

Scheidt, C., Caers, J., 2009. Representing spatial uncertainty using distances and kernels. Mathematical Geosciences 41 (4), 397-419.

Scheidt, C., Caers, J., Chen, Y., Durlofsky, L. J., 2011. A multi-resolution workflow to generate high-resolution models constrained to dynamic data. Computational Geoscience, doi: 10.1007/s10596-011-9223-9.

Schöniger, A., 2010. Parameter estimation by ensemble Kalman filters with transformed data. Master's thesis, University of Stuttgart. 95pp.

Schöniger, A., Nowak, W., Hendricks Franssen, H. J., 2011. Parameter estimation by ensemble Kalman filters with transformed data: approach and application to hydraulic tomography. Water Resources Research, under review.

Slooten, L., Carrera, J., Castro, E., Fernandez-Garcia, D., 2010. A sensitivity analysis of tide-induced head fluctuations in coastal aquifers. Journal of Hydrology 393 (3-4), 370-380. 
Strebelle, S., 2002. Conditional simulation of complex geological structures using multiple-point statistics. Mathematical Geology 34 (1), 1-21.

Sun, N.-Z., 1994. Inverse problems in groundwater modeling. Kluwer Academic, Dordrecht. 337pp.

Suzuki, S., Caers, J., 2008. A distance-based prior model parameterization for constraining solutions of spatial inverse problems. Mathematical Geosciences 40 (4), 445-469.

Wen, X., Chen, W., 2005. Real-time reservoir model updating using ensemble Kalman filter. SPE 92991.

Wen, X., Gómez-Hernández, J. J., 1996. Upscaling hydraulic conductivities: An overview. Journal of Hydrology 183 (1-2), ix-xxxii.

Wen, X., Lee, S., Yu, T., 2006. Simultaneous integration of pressure, water cut, 1 and 4-D seismic data in geostatistical reservoir modeling. Mathematical Geology 38 (3), 301-325.

Wen, X. H., Capilla, J. E., Deutsch, C. V., Gómez-Hernández, J. J., Cullick, A. S., 1999. A program to create permeability fields that honor single-phase flow rate and pressure data. Computers \& Geosciences 25 (3), 217-230.

Wen, X. H., Deutsch, C. V., Cullick, A. S., 2002. Construction of geostatistical aquifer models integrating dynamic flow and tracer data using inverse technique. Journal of Hydrology 255 (1-4), 151-168.

Woodbury, A. D., Ulrych, T. J., 1993. Minimum relative entropy: Forward probabilistic modeling. Water Resources Research 29 (8), 2847-2860.

Yeh, W. W., 1986. Review of parameter identification procedures in groundwater hydrology: The inverse problem. Water Resources Research 22 (2), $95-108$.

Ying, Z., Gomez-Hernandez, J., 2000. An improved deformation algorithm for automatic history matching: Report 13. Stanford Center for Reservoir Forecasting (SCRF) Annual Report, Stanford, CA.

Zhou, H., Gómez-Hernández, J. J., Hendricks Franssen, H.-J., Li, L., 2011a. An approach to handling Non-Gaussianity of parameters and state variables in ensemble Kalman filter. Advances in Water Resources 34 (7), 844-864.

Zhou, H., Gómez-Hernández, J. J., Li, L., 2011b. A pattern search based inverse method. Water Resources Research, under review. 
Zhou, H., Li, L., Gómez-Hernández, J. J., 2010. Three-dimensional hydraulic conductivity upscaling in groundwater modeling. Computers \& Geosciences 36 (10), 1224-1235.

Zimmerman, D. A., Marsily, G. D., Gotway, C. A., Marietta, M. G., Axness, C. L., Beauheim, R. L., Bras, R. L., Carrera, J., Dagan, G., Davies, P. B., et al., 1998. A comparison of seven geostatistically based inverse approaches to estimate transmissivities for modeling advective transport by groundwater flow. Water Resources Research 34 (6), 1373-1413.

Zinn, B., Harvey, C., 2003. When good statistical models of aquifer heterogeneity go bad: A comparison of flow, dispersion, and mass transfer in connected and multivariate Gaussian hydraulic conductivity fields. Water Resources Research 39 (3), 1051. 


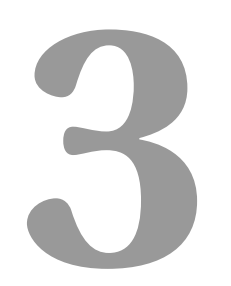

\title{
An Approach to Handling Non-Gaussianity of Parameters and State Variables in Ensemble Kalman Filtering
}

\begin{abstract}
The ensemble Kalman filter (EnKF) is a commonly used real-time data assimilation algorithm in various disciplines. Here, the EnKF is applied, in a hydrogeological context, to condition log-conductivity realizations on logconductivity and transient piezometric head data. In this case, the state vector is made up of log-conductivities and piezometric heads over a discretized aquifer domain, the forecast model is a groundwater flow numerical model, and the transient piezometric head data are sequentially assimilated to update the state vector. It is well known that all Kalman filters perform optimally for linear forecast models and a multiGaussian-distributed state vector. Of the different Kalman filters, the EnKF provides a robust solution to address non-linearities; however, it does not handle well non-Gaussian state-vector distributions. In the standard EnKF, as time passes and more state observations
\end{abstract}


are assimilated, the distributions become closer to Gaussian, even if the initial ones are clearly non-Gaussian. A new method is proposed that transforms the original state vector into a new vector that is univariate Gaussian at all times. Back transforming the vector after the filtering ensures that the initial non-Gaussian univariate distributions of the state-vector components are preserved throughout. The proposed method is based in normal-score transforming each variable for all locations and all time steps. This new method, termed the normal-score ensemble Kalman filter (NS-EnKF), is demonstrated in a synthetic bimodal aquifer resembling a fluvial deposit, and it is compared to the standard EnKF. The proposed method performs better than the standard EnKF in all aspects analyzed (log-conductivity characterization and flow and transport predictions).

\subsection{Introduction}

Groundwater modeling and prediction plays a critical role in decision making for groundwater management and environmental protection. In order to make reliable groundwater flow model predictions, it is necessary to account for all measured data. Although important information can be obtained from field work, it is in practice impossible to characterize an aquifer exhaustively. To best account for the state information - such as flows, hydraulic heads or concentrations - in the characterization of aquifer parameters, numerous methods of parameter identification have been proposed (e.g., for an overview see Carrera et al., 2005; De Marsily et al., 2000; Hendricks Franssen et al., 2009; McLaughlin and Townley, 1996; Oliver and Chen, 2010; Yeh, 1986).

In the early days of parameter identification, the aim was to obtain a single "best" estimate of the aquifer parameters. However, it has been proven that such a "best" estimate is always much smoother than the real aquifer and that flow and transport predictions performed in such estimate are very poor (Gómez-Hernández and Wen, 1994). The alternative is to resort to Monte Carlo analysis in which multiple realizations of the aquifer parameters are built accounting for all the measured data. Each realization represents a possible case of the unknown reality. Flow and transport are modeled in each realization and all model predictions are collected to characterize uncertainty and to define an optimal prediction (e.g., pilot point method (Alcolea et al., 2006; RamaRao et al., 1995), self-calibration (Gómez-Hernández et al., 1997) and gradual deformation $(\mathrm{Hu}, 2000)$ ).

One such Monte Carlo-based method is the ensemble Kalman filter (EnKF) proposed by Evensen (1994) and subsequently clarified by Burgers et al. (1998). The EnKF is an extension of the Kalman filter to deal with nonlinear state equations. The Kalman filter (Kalman, 1960) proved to be a very powerful 
data assimilation algorithm for systems in which the relation between parameters and state is linear. This linearity allowed an exact propagation of the state covariance in time. The first attempt to deal with nonlinear transfer functions, such is the case in groundwater modeling, was the extended Kalman filter (e.g., Leng and Yeh, 2003; Yeh and Huang, 2005). In the extended Kalman filter, the nonlinear transfer function is linearized after a Taylor expansion and this linearization is used for the covariance propagation. For highly nonlinear transfer functions, extended Kalman filter tends to deteriorate as time progresses, since the errors in the covariance propagation accumulate; at the same time, extended Kalman filter is time consuming when the aquifer is finely discretized (Evensen, 2003).

The EnKF circumvents the problem of covariance propagation in time by working with an ensemble of realizations. In each realization the state equation is solved, and the ensemble of states is used to compute the covariance explicitly and efficiently. The EnKF has gained popularity in diverse disciplines such as oceanography, meteorology and hydrology (e.g., Bertino et al., 2003; Chen and Zhang, 2006; Houtekamer and Mitchell, 2001; Li et al., 2011b; Moradkhani et al., 2005; Nowak, 2009; Wen and Chen, 2005a). The advantages of the EnKF can be summarized as follows: first, CPU consumption is reduced mostly because of the way the state covariance is computed (Hendricks Franssen and Kinzelbach (2009) documented a reduction of the needed CPU time by a factor of 80 as compared with other Monte-Carlo type inverse modeling); second, the EnKF can easily be combined with different forecast models; third, the EnKF is capable of incorporating the observations sequentially in time without the need to store all previous states. In addition, although Evensen (1994) developed the EnKF to obtain a single optimal estimate of the system state, the EnKF provides, as a by-product, the entire ensemble of states, which can be used to assess uncertainty.

Our objective with this paper is to use the EnKF for the generation of an ensemble of hydraulic conductivity realizations which are conditional to hydraulic conductivity measurements and to piezometric head data. This approach has already been described in the literature, for instance by Hendricks Franssen and Kinzelbach (2008), who generated realizations of transmissivity and recharge, and by Liu et al. (2008), who focused on hydraulic conductivity, dispersivity, mass transfer rate and mobile porosity ratio for a dual-domain mass transfer model at the MADE site. Example applications in petroleum engineering can be found in the works by Wen and Chen (2005b) and Gu and Oliver (2007). Our contribution is to develop a new approach applicable to non-Gaussian distributions of hydraulic conductivities, with an application to a bimodal distribution typical, for instance, of fluvial deposits.

It can be shown that the EnKF provides an optimal solution when the parameter vector follows a multiGaussian distribution and the state transfer 
function is linear (Arulampalam et al., 2002). In most practical applications in groundwater and petroleum engineering neither the parameters can be modeled as multiGaussian nor the transfer function is linear. The importance of accounting for the nonGaussianity of hydraulic conductivity has already been demonstrated in the literature (Gómez-Hernández and Wen, 1998; Journel and Deutsch, 1993; Zinn and Harvey, 2003). To circumvent the problems of the EnKF, some authors have concentrated in the problem of non-Gaussianity in the parameters and others have focused on reparameterizing the state equation so that the relationship between model parameters and state variables is closer to linear. Sun et al. (2009) worked on the non-Gaussianity aspect and took advantage of localization techniques with a Gaussian mixture model to update the parameters of a multimodal distribution. Chen et al. (2009) addressed the nonlinearity problem by reparameterization.

In this work we take the route of transforming parameters and state variables so that they both follow marginal Gaussian distributions. These transformations, which are themselves highly nonlinear, will make the state transfer function even more nonlinear than for the untransformed variables, but, in return, the Kalman filtering equations will be applied on Gaussian variates. We demonstrate this approach on a synthetic aquifer resembling a highly channelized fluvial deposit. It will be shown how this approach improves the results obtained using a standard implementation of the EnKF even though the univariate transformation applied ensures marginal Gaussian distributions but does not ensure multiGaussianity of the joint state vector.

We are aware that in another study (Schöniger et al., 2011) similar transformation techniques as proposed in this paper were applied but only to the state variables, not to the parameters, in the context of hydraulic tomography. The authors focus on the improvements that can be achieved with different transformation techniques, analyze under which conditions improvements can be obtained, and demonstrate a pseudo-linearizing effect of their transformations. For reference, they compared their improved method to an exhaustive particle filter. Similar applications can be found in other disciplines; for instance, in reservoir modeling, transformation from non-Gaussian distribution to Gaussian distribution is applied to the state variables, such as saturation, by $\mathrm{Gu}$ and Oliver (2006) and, in ocean ecosystem modeling, a similar transformation is applied to chlorophyll- $a$ concentration by Simon and Bertino (2009). Other transformation algorithms can be found in the literature such as in Béal et al. (2010) and Bocquet et al. (2010). In contrast with all of these applications, the method proposed in this paper focuses on transforming not only the non-Gaussian distributed state variables but, most importantly, the non-Gaussian distributed model parameters, i.e., the hydraulic conductivities, which are commonly assumed to follow a log-normal distribution. These transformation of parameters and state variables will make the relationship between 
the transformed variables even more nonlinear (instead of more linear as pursued in the previously cited works), but the Kalman filtering equations will be applied to Gaussian variables. To our knowledge, no such transformation algorithm has been applied to handle the non-Gaussian distribution of hydraulic conductivities in the scope of the EnKF in hydrogeology.

Throughout the paper we use interchangeably the terminology from geostatistics, hydrogeology and Kalman filtering, most noticeably, (a) piezometric head data assimilation is equivalent to (inverse) data conditioning, in the sense that the solution of the flow equation in each of the final realizations of hydraulic conductivity will match the measured piezometric heads, (b) by forecast model, transfer function or state transition model we refer to the transient groundwater flow equation and its corresponding numerical model, and (c) when referring to the flow equation we distinguish between parameters (i.e., hydraulic conductivities) and state variables (i.e., piezometric heads), whereas in the EnKF we will talk about a (joint) state vector that includes realizations of both parameters and state variables at time $t$. Also, since our goal is the characterization of the hydraulic conductivity spatial variability, we will be using the term hard data to refer to local measurements of hydraulic conductivity, as opposed to measurements of piezometric heads which are soft data since they do not measure directly hydraulic conductivity but serve to characterize its spatial variability.

The rest of the paper is structured as follows. After the standard EnKF is introduced, the new algorithm is explained in detail. Then a synthetic bimodal aquifer is used to evaluate the performance of the proposed method. The paper ends with a discussion and some conclusions.

\subsection{Methodology}

We first present the groundwater flow and transport equations that will be used in the synthetic example, then we follow with the description of the standard EnKF and propose the new algorithm with the transformed variables, which will be referred to as the normal score EnKF (NS-EnKF). The flow model will be used in conjunction with ensemble Kalman filtering to obtain realizations of hydraulic conductivity conditioned to both conductivity and piezometric head data. The transport model will be used only for verification purposes to evaluate how well transport is predicted in the final conductivity fields. 


\subsubsection{Flow and transport equations}

By combining mass conservation and Darcy's law, the groundwater flow equation in saturated porous media can be expressed as (Bear, 1972):

$$
\nabla \cdot(K \nabla h)=S_{s} \frac{\partial h}{\partial t}+Q
$$

subject to initial and boundary conditions. In the differential equation, $\nabla$. is the divergence operator, $\nabla$ is the gradient operator, $K$ is the hydraulic conductivity $\left[L T^{-1}\right], h$ represents hydraulic head $[L], S_{s}$ is specific storage $\left[L^{-1}\right], Q$ is the source-sink term $\left[T^{-1}\right]$ and $t$ is time $[T]$. Equation 3.1 is numerically solved by applying a discretization in space and time.

The governing equation for non-reactive transport in the subsurface is:

$$
\phi \frac{\partial C}{\partial t}=-\nabla \cdot(q C)+\nabla \cdot(\phi \mathbf{D} \nabla C)
$$

subject to initial and boundary conditions, where $C$ is the concentration of solute in the liquid phase $\left[M L^{-3}\right] ; \phi$ is porosity [-]; $\mathbf{D}$ is the local hydrodynamic dispersion coefficient tensor $\left[L^{2} T^{-1}\right]$ with principal axes parallel and perpendicular to the direction of flow and eigenvalues proportional to the components of the fluid velocity and the longitudinal and transverse dispersivities, and $q$ is the Darcy velocity $\left[L T^{-1}\right]$ calculated by Darcy's law: $q=-K \nabla h$.

\subsubsection{Standard EnKF}

The theory and numerical implementation of the EnKF is described extensively in Evensen $(2003,2007)$. Here we will only recall that the EnKF deals with dynamic systems, for which state data are observed as a function of time and used to sequentially update the parameters and the state of the system. For this purpose an ensemble of realizations is generated and then each realization is updated as observation data are available.

In the EnKF, the state vector $\mathbf{x}$ is represented by the collection of all state vectors for all $N_{r}$ realizations, $\mathbf{x}=\left(x_{1}, x_{2}, \ldots, x_{N_{r}}\right)$. For realization $i, x_{i}$ includes both the parameters controlling the transfer function and the state variables:

$$
x_{i}=\left(\begin{array}{l}
A \\
B
\end{array}\right)_{i}=\left(\begin{array}{c}
\left(a_{1}, a_{2}, \ldots, a_{N \cdot N_{p}}\right)^{\mathrm{T}} \\
\left(b_{1}, b_{2}, \ldots, b_{N \cdot N_{s}}\right)^{\mathrm{T}}
\end{array}\right)_{i}
$$

where $A$ is the vector of model parameters, such as hydraulic conductivities and porosities, and $B$ is the vector with the state variables, such as hydraulic heads and concentrations. The size of the state vector for each realization is determined by the number of grid cells in which the domain has been discretized $(N)$, the number of model parameters $\left(N_{p}\right)$, and the number of state 
variables $\left(N_{s}\right)$. In our case, only one type of parameter (log-hydraulic conductivity $\ln K$ ) and one type of state variable (piezometric head $h$ ) are considered, therefore

$$
x_{i}=\left(\begin{array}{c}
\left(\ln K_{1}, \ln K_{2}, \ldots, \ln K_{N}\right)^{\mathrm{T}} \\
\left(h_{1}, h_{2}, \ldots, h_{N}\right)^{\mathrm{T}}
\end{array}\right)_{i} .
$$

The final size of the ensemble state vector is equal to the size of each realization state vector multiplied by the number of realizations. In our case, it is $2 \times$ $N \times N_{r}$

The EnKF consists of two main steps: a forecast step and an update step. Both steps are to be performed in each realization. The forecast step involves the transition of the state variables from time $t-1$ to time $t$,

$$
\mathbf{x}_{t}=F\left(\mathbf{x}_{t-1}\right)
$$

where $F(\cdot)$ is the transfer function. In our case this transfer function leaves the log-conductivities unchanged and forecasts the piezometric heads to the next time step using the transient groundwater flow model (Equation 3.1).

After data are collected, the state vector is updated by the EnKF. The update process is summarized by the following equations:

$$
\begin{aligned}
\mathbf{x}_{t}^{u} & =\mathbf{x}_{t}^{f}+\mathbf{G}_{t}\left(\mathbf{z}_{t}+\varepsilon-\mathbf{H} \mathbf{x}_{t}^{f}\right) \\
\mathbf{G}_{t} & =\mathbf{P}_{t}^{f} \mathbf{H}^{\mathrm{T}}\left(\mathbf{H P}_{t}^{f} \mathbf{H}^{\mathrm{T}}+\mathbf{R}\right)^{-1} \\
\overline{\mathbf{x}}_{t}^{f} & \approx \frac{1}{N_{r}} \sum_{i=1}^{N_{r}} x_{t, i}^{f} \\
\mathbf{P}_{t}^{f} & \approx \frac{1}{N_{r}}\left(\mathbf{x}_{t}^{f}-\overline{\mathbf{x}}_{t}^{f}\right)\left(\mathbf{x}_{t}^{f}-\overline{\mathbf{x}}_{t}^{f}\right)^{\mathrm{T}}
\end{aligned}
$$

where $\mathbf{x}_{t}^{u}$ is the vector with the updated model parameters and state variables at time $t$ and $\mathbf{x}_{t}^{f}$ is the vector with the forecasted state; $\mathbf{G}_{t}$ is the Kalman gain - derived after the minimization of a posterior error covariance - it multiplies the residuals between observed and forecasted values to provide an update to the latter; $\mathbf{z}_{t}$ are the observations at time $t ; \varepsilon$ is an observation error, generally characterized by a normal distribution with zero mean and a diagonal covariance $\mathbf{R}$ (we assume that errors at different measurement locations are independent); $\mathbf{H}$ is the observation matrix which has as many rows as number of measurements $(M)$ and in our case consists of 0 's and 1's since we assume that measurement locations coincide with the grid nodes; $x_{t, i}^{f}$ is a realization of the ensemble of forecasted state vectors; $\overline{\mathbf{x}}_{t}^{f}$ is the ensemble mean; and $\mathbf{P}_{t}^{f}$ is the ensemble covariance matrix of the state $\mathbf{x}_{t}^{f}$. It is worth noting that we do not have to compute the whole covariance matrix explicitly because we can compute directly $\mathbf{P}_{t}^{f} \mathbf{H}^{\mathrm{T}}$ and $\mathbf{H} \mathbf{P}_{t}^{f} \mathbf{H}^{\mathrm{T}}$ taking advantage of the fact that most of the entries of $\mathbf{H}$ are zeroes. 
The EnKF has an intrinsic feature in honoring parameter data (i.e., hydraulic conductivity measurements) as long as they remain constant during the forecast step and parameter measurement errors are neglected. In such a case the components of the covariance matrix $\mathbf{P}_{t}^{f}$ involving parameter measurement locations are equal to zero, the Kalman gain at those locations is also zero, and therefore they remain constant also through the update step.

\subsubsection{Normal-score EnKF}

As we have mentioned before, we are going to modify the formulation of the standard EnKF so that we ensure that the joint state vector follows marginal Gaussian distributions at all locations and all times. This is achieved by applying a normal-score transform, independently, to each element of the state vector as discussed in the appendix A. This normal-score transform function (TF) has to be recomputed after each forecast step. Let $\Phi_{t}(\cdot)$ represent the normal-score transformation at time $t$ so that

$$
\mathbf{y}_{t}=\Phi_{t}\left(\mathbf{x}_{\mathbf{t}}\right)
$$

is a new state vector in which all variables have a marginal Gaussian distribution with zero mean and unit variance. Similarly we will have a normally distributed state vector at time $t-1, \mathbf{y}_{t-1}=\Phi_{t-1}\left(\mathbf{x}_{\mathbf{t}-\mathbf{1}}\right)$. Substituting $\mathbf{y}$ for $\mathbf{x}$ in the transfer function (3.5) we obtain

$$
\mathbf{y}_{t}=\Phi_{t}\left(F\left(\Phi_{t-1}^{-1}\left(\mathbf{y}_{t-1}\right)\right)\right),
$$

which we can rewrite as

$$
\mathbf{y}_{t}=\varphi_{t}\left(\mathbf{y}_{t-1}\right)
$$

with $\varphi_{t}=\Phi_{t} \cdot F \cdot \Phi_{t-1}^{-1}$. We have replaced the original nonlinear transfer function $F(\cdot)$ with a new nonlinear transfer function $\varphi_{t}(\cdot)$ that takes as input a vector of Gaussian variables and propagates it in time into a new vector which is also Gaussian. (Notice that the normal score transform only renders the variables Gaussian one by one, the multivariate properties of the state vector are also changed but not necessarily to become multiGaussian.)

The inverse of the normal-score transform function permits, at any time step and for any location, to retrieve the state vector $\mathbf{x}$ from the vector $\mathbf{y}$ ensuring that the marginal non-Gaussian distribution of $\mathbf{x}$ is kept. This is particularly interesting in cases such as aquifer modeling in fluvial deposits, which are characterized by multimodal distributions of log-conductivities.

Once the new transfer function in Equation 3.9 is established, the NSEnKF just follows the same steps as the standard EnKF. However, since the transfer function depends on the normal-score transform function that, in turn, depends on the state values, there is not an explicit expression. A flow chart of the NS-EnKF is displayed in Figure 3.1 which consists of the following steps: 


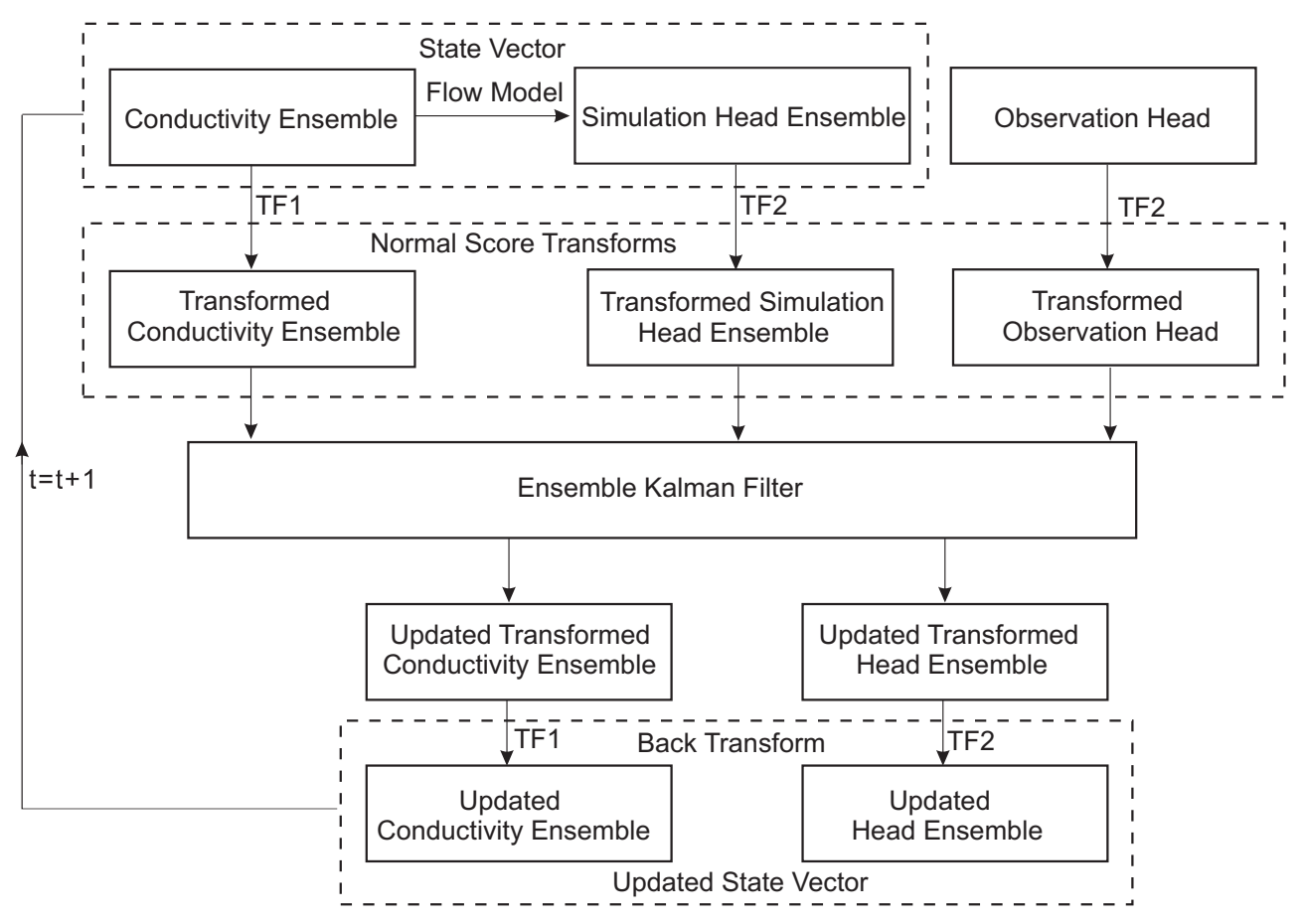

Figure 3.1: Flow chart of the NS-EnKF. 
1. Generate the initial ensemble. A large number of equally likely stochastic realizations of the state vector are generated. In our case the state vector consists of log-conductivities and piezometric heads; the log-conductivities are generated using geostatistical simulation techniques (Deutsch and Journel, 1998), which are conditioned to hard data; the piezometric heads are set equal to the initial heads, which in our case are zero everywhere (alternatively the initial heads could be set equal to the steadystate solution of the flow equation for given boundary conditions and external sources, or they could be obtained from a warm-up run).

2. Forecast. For each realization, the log-conductivities stay unchanged, and the piezometric heads are the solutions of the groundwater flow equation in each realization from time $t-1$ to time $t$.

3. Normal-score transform. Establish the local cumulative distribution functions (CDFs) for all the components of the state vector from the ensemble of realizations. In our case there will be one such local CDF at each location for the log-conductivity and another one for the piezometric head. Use these local CDFs to build the normal-score transformation function and transform the state vector into a new vector, with all its components following marginal Gaussian distributions with zero mean and unit variance. It is worthwhile to mention that the log-conductivities transform functions remain unchanged during the data assimilation (TF1 in Figure 3.1); this helps recovering the prior model structure. On the contrary, the transformation functions for piezometric heads have to be recomputed at each time step (TF2 in Figure 3.1).

4. Update. State data are collected at time $t$. These data are normal-score transformed using the same local transformation functions computed in the previous step. In our example we only collect piezometric head data although the method would be applicable if log-conductivity data were collected, too. Next, we apply Equation 3.6 to update the state vector.

5. Back transform. The updated state vector is back transformed using the previously constructed transformation functions. Time advances one step, the updated state vector becomes the current vector and we loop back to the forecast step.

To sum up, the proposed method applies the EnKF always to a state vector all of which components follow a marginal Gaussian distribution. Furthermore, using the normal-score transformation we ensure that the prior nonGaussian marginals of the model parameters are kept throughout, in our case, the prior bimodal pattern of the log-conductivities. We recognize that, in the proposed method, the model parameters and state variables are transformed 
Table 3.1: Scenario definition. Parameters that change from one scenario to another.

\begin{tabular}{cccccc}
\hline Scenario & No. hard data & Variance & No. realizations & EnKF & NS-EnKF \\
\hline 1 & 80 & 1.72 & 1000 & $\times$ & \\
2 & 80 & 1.72 & 1000 & & $\times$ \\
3 & 80 & 1.72 & 200 & & $\times$ \\
4 & 20 & 1.72 & 1000 & & $\times$ \\
5 & 80 & 9.92 & 1000 & & $\times$ \\
\hline
\end{tabular}

into Gaussian space independently of each other, which yields a state vector with univariate Gaussian marginal distribution but not necessarily jointly multiGaussian.

\subsection{Synthetic experiment}

Aquifers of fluvial deposits are a typical example of geologic formations in which conductivities have a non-Gaussian distribution. Moreover, the hydraulic conductivities in such aquifers typically show a variation over several orders of magnitude. In this section, a synthetic bimodal aquifer composed of sand and clay is built to demonstrate the effectiveness of the proposed method. The NS-EnKF is compared with the standard EnKF in order to investigate whether and to what extent the normal-score transformation improves aquifer characterization for non-Gaussian cases. The influence of several parameters (i.e., number of realizations, number of hard data and magnitude of hydraulic conductivity variance) is investigated (see Table 3.1 for the description of the different scenarios).

Direct sampling, a multiple-point geostatistical simulation algorithm, is used to generate facies-distribution realizations (Mariethoz et al., 2010). Direct sampling is a pattern-based geostatistic simulation approach that generates realizations by borrowing structures from a training image. A training image generated by FLUVSIM (Deutsch and Tran, 2002) serves as a conceptual model of the bimodal aquifer composed of sand with high permeability and floodplain fine-grid deposits (e.g., clay) with low permeability (see Figure 3.2). Compared with traditional variogram-based two-point geostatistics, multiple-point geostatistics is well suited for handling curvilinear structures, which cannot be characterized well with a variogram.

The numerical experiment is carried out for a synthetic aquifer of $1 \mathrm{~m}$ thickness extending over a domain of $500 \mathrm{~m} \times 400 \mathrm{~m}$ discretized in $2 \mathrm{D}$ into 100 columns by 80 rows (i.e., square grid cells of $5 \mathrm{~m}$ ). The reference facies field (Figure 3.3A) is generated by direct sampling based on the training image of 


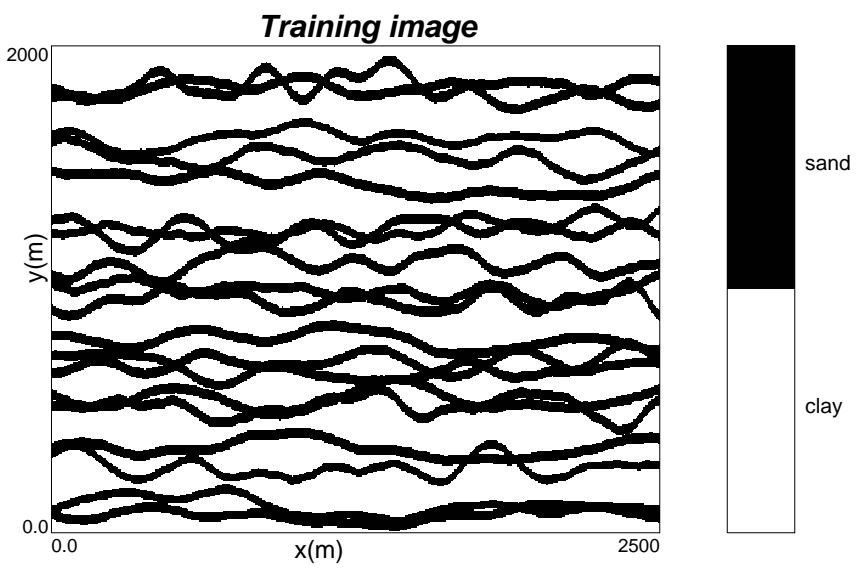

Figure 3.2: Training image used to generate the ensemble of binary facies realizations.

Figure 3.2. Then, each facies is populated with log-conductivity values. These log-conductivity values are generated by GCOSIM3D (Gómez-Hernández and Journel, 1993), independently for each facies, according to stationary multiGaussian random functions with parameters given in Table 3.2. The reference $\ln K$ field is plotted in Figure 3.3B. The histogram of $\ln K$ (Figure 3.3E) shows a pronounced bimodality with a global mean of $-4.43 \ln (\mathrm{cm} / \mathrm{s})$ and variance of $1.72(\ln (\mathrm{cm} / \mathrm{s}))^{2}$. Hydraulic conductivity measurements are taken from this reference field at the locations shown in Figure 3.3C. These data will be used as conditioning hard data.

Table 3.2: Parameters defining the multiGaussian random function used to generate the log-conductivity within each of the two facies.

\begin{tabular}{ccllcc}
\hline Facies & Variogram type & $\begin{array}{l}\text { Mean } \\
(\ln (\mathrm{cm} / \mathrm{s}))\end{array}$ & $\begin{array}{l}\text { Std.dev. } \\
(\ln (\mathrm{cm} / \mathrm{s}))\end{array}$ & $\lambda_{x}^{*}(\mathrm{~m})$ & $\lambda_{y}^{*}(\mathrm{~m})$ \\
\hline clay & exponential & -5.5 & 0.5 & 50 & 50 \\
sand & exponential & -3.0 & 0.5 & 100 & 50 \\
\hline
\end{tabular}

* ranges in the $x$ and $y$ directions.

The groundwater flow equation under confined conditions is solved for the reference hydraulic conductivity field assuming impermeable boundary conditions on the northern and southern boundaries, constant prescribed head on the western boundary equal to zero meters and prescribed specific discharge through the eastern boundary of $-2.2 \mathrm{~m} / \mathrm{d}$ (Figure 3.3D). The initial head is $0 \mathrm{~m}$ over the area of interest. The groundwater flow equation (Equation 3.1 ) is solved by finite differences in both space and time using the simulator 

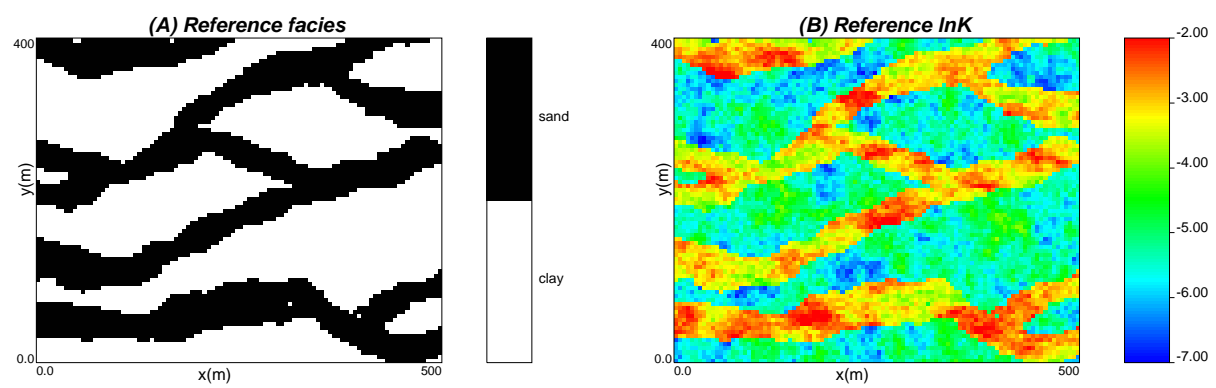

(D) Boundary conditions and well configuration
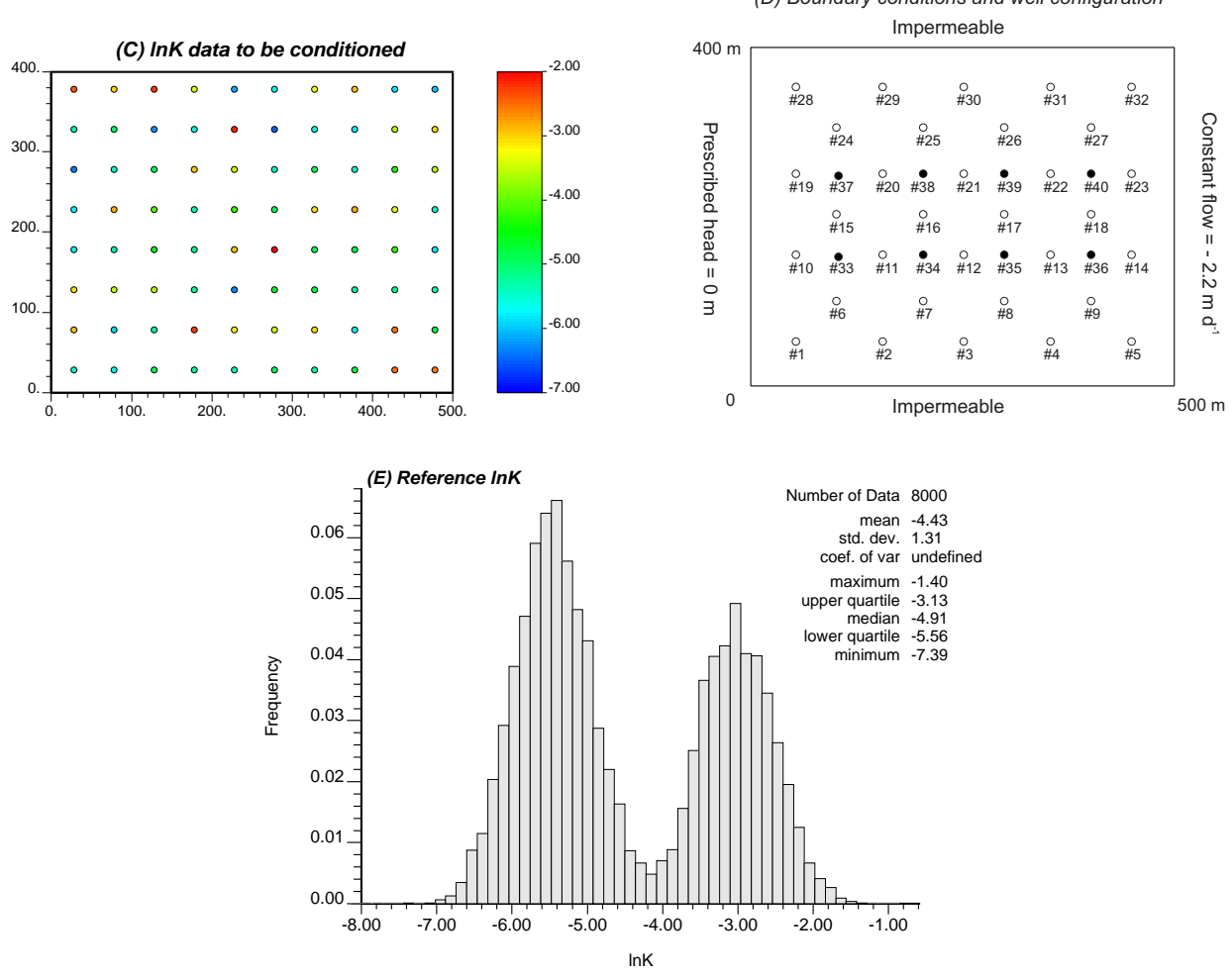

Figure 3.3: (A) Reference facies field; (B) reference log-conductivity field $(\ln (\mathrm{cm} / \mathrm{s})) ;(\mathrm{C})$ hydraulic conductivity measurements used for conditioning; (D) flow boundary conditions and location of piezometers (open circles for observation, solid circles for prediction); (E) histogram of reference logconductivity. 
developed by $\mathrm{Fu}$ (2008). The simulation period of 300 days is discretized into 100 time steps, the duration of which follows a geometric series with ratio of 1.05. There are no internal sinks or sources in this example. Specific storage is assumed constant and equal to $0.003 \mathrm{~m}^{-1}$. Piezometers cover the aquifer regularly (see Figure 3.3D), and the transient evolution of the piezometric heads in the reference field at these locations is sampled. Head observations from piezometers \#1 to \#32 will be used as observation data (conditioning data) during the update step of the NS-EnKF. The remaining piezometers will be used for validation purposes.

Similarly as we did to generate the reference realization, one thousand facies realizations are generated by direct sampling using the training image depicted in Figure 3.2. Then, in each realization, both facies are populated with $\ln K$ values generated by sequential Gaussian simulation. It is important to stress that both the facies realizations and the log-conductivity realizations are conditional to the hard data sampled from the reference field and shown in Figure 3.3C. The geostatistical parameters used in the sequential simulation are the same as for the reference given in Table 3.2. By generating the realizations in this way, neither statistical model uncertainty nor log-conductivity measurement uncertainty are considered in this example. For illustration purposes, a randomly selected realization is shown in Figure 3.4 together with the ensemble mean of the 1000 realizations.
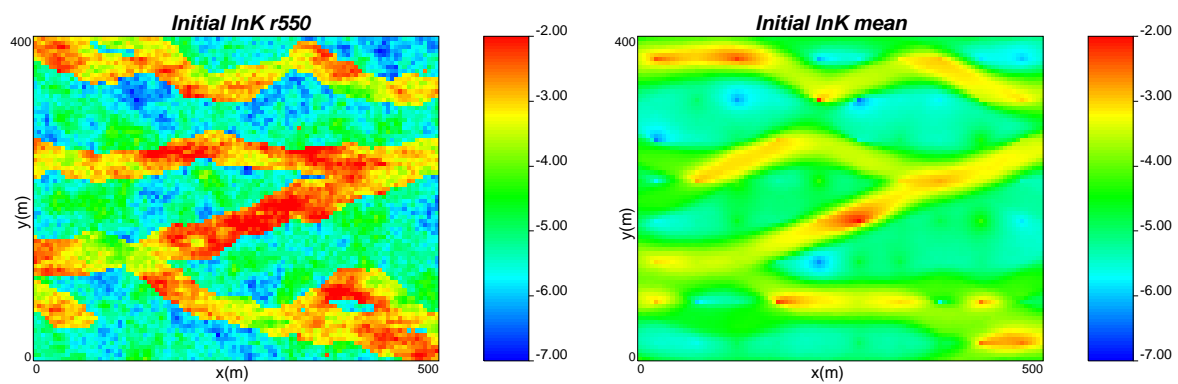

Figure 3.4: A randomly selected $\ln K$ realization $\left(550^{\text {th }}\right)$ from the initial ensemble and the ensemble mean of all 1000 initial realizations for Scenarios 1 and 2 .

Next, hydraulic head data assimilation by the NS-EnKF is performed as illustrated in section 2.3. The transient hydraulic heads observed in the reference field during the first 50 time steps (22.8 days) at the 32 monitoring piezometers are used to update the state vector after each time step. The observed heads during the remaining 50 time steps are used for model verification. Head observation error is characterized by a normal distribution with zero mean and a standard deviation of $0.01 \mathrm{~m}$. 
Recall that the term assimilation, commonly used in the filtering literature, is equivalent to the term conditioning used in the geostatistical literature.

\subsection{Performance assessment measures}

Ensemble means and ensemble standard deviations will be computed. The ensemble mean map should reproduce the main spatial patterns of hydraulic conductivity, as close as permitted by the conditioning data set, and it will be visually compared with the reference map to get a qualitative assessment. The standard deviation map will indicate, locally, the degree of uncertainty and, as will be seen, will serve to delineate the edges of the channels. For a quantitative assessment we compute a precision measure given by the Average Absolute Deviation $\left(A A D(x)_{t}\right)$, and, taking advantage of knowing the reference, we also compute a bias measure, the Average Absolute Error $\left(A A E(x)_{t}\right)$.

$$
\begin{aligned}
& A A D(x)_{t}=\frac{1}{N_{x y}} \frac{1}{N_{r}} \sum_{i=1}^{N x y} \sum_{j=1}^{N_{r}}\left|x_{t, i, j}-\bar{x}_{t, i}\right| \\
& A A E(x)_{t}=\frac{1}{N_{x y}} \sum_{i=1}^{N_{x y}}\left|\bar{x}_{t, i}-x_{t, i}^{r e f}\right|
\end{aligned}
$$

where $x_{t, i, j}$ is the state at time step $t$, node $i$ and realization $j, N_{x y}$ and $N_{r}$ are the number of grid cells and realizations, respectively, $\bar{x}_{t, i}$ represents ensemble mean at time $t$ and node $i$, and $x_{t, i}^{r e f}$ is the reference state at time $t$ and node $i$.

The histograms of hydraulic conductivity before and after data assimilation will be compared.

Connectivity of conductivity plays a critical role for transport: early arrival times of contaminants are very sensitive to the existence of channels characterized by connected high-conductivity values, and late arrival times are influenced by the presence of barriers of connected low-conductivity values. A series of measures have been proposed to evaluate connectivity (Knudby and Carrera, 2005) and we adopt the connectivity function as defined by Stauffer and Aharony (1994) because it is straightforward and easy to compute. This connectivity function gives the probability that two points are connected within the same facies by a continuous path. Thus, connectivity is a nonincreasing function of Euclidean distance. In our case, in order to calculate the connectivity function, the continuous log-conductivity fields are converted to indicator fields with values 0 and 1 :

$$
I(x)= \begin{cases}1, & \text { if } \ln K \geq-4 \\ 0, & \text { otherwise }\end{cases}
$$


where the threshold of -4 splits, approximately, the log-conductivities into sand and clay (Figure 3.3E). We use the program CONNEC3D (Pardo-Igúzquiza and Dowd, 2003) to calculate the probability that two points with log-conductivities larger than -4 are connected following a continuous path with log-conductivities larger than -4 .

Hydraulic conductivity and piezometric head conditioning is not enough to ensure good transport predictions (Meier et al., 2001). We analyze the transport prediction ability of the updated conductivity fields by performing a transport experiment - once piezometric head is at steady state - in which particles are uniformly released over a line source at $x=17 \mathrm{~m}$. The integrated breakthrough curves (BTCs) at a control plane positioned at $x=$ $450 \mathrm{~m}$ are computed in the reference field and compared to the BTCs in the updated fields (see Figure 3.5 for the experiment setup). Average Absolute Error $A A E\left(T_{\alpha}\right)$ and Average Absolute Deviation $A A D\left(T_{\alpha}\right)$ are computed for different percentiles of the cumulative mass distribution.

$$
\begin{aligned}
& A A E\left(T_{\alpha}\right)=\frac{1}{N_{r}} \sum_{r=1}^{N_{r}}\left|T_{r, \alpha}-T_{r e f, \alpha}\right| \\
& A A D\left(T_{\alpha}\right)=\frac{1}{N_{r}} \sum_{r=1}^{N_{r}}\left|T_{r, \alpha}-\bar{T}_{\alpha}\right|
\end{aligned}
$$

where $T_{r, \alpha}$ is the travel time for the $r^{\text {th }}$ realization, the $\alpha$ percentile of the cumulative mass distribution ( $\alpha$ values of $5 \%, 50 \%$ or $95 \%$ are analyzed), $T_{r e f, \alpha}$ refers to the travel times corresponding to the reference field, and $\bar{T}_{\alpha}$ represents mean travel time over the ensemble.

\subsection{Results and discussion}

\subsubsection{Standard EnKF versus NS-EnKF}

In this subsection we will show that conditioning to piezometric head data by either the EnKF or the NS-EnKF is always beneficial, since either method produces a final ensemble of realizations that better characterizes the spatial variability of hydraulic conductivity than the initial ensemble of realizations. We will also show that the NS-EnKF outperforms the standard EnKF for the fluvial aquifer used here both with regard to the characterization of hydraulic conductivity, and in the capabilities of the updated hydraulic conductivity model to predict piezometric heads at unsampled locations, and mass transport through the aquifer. 


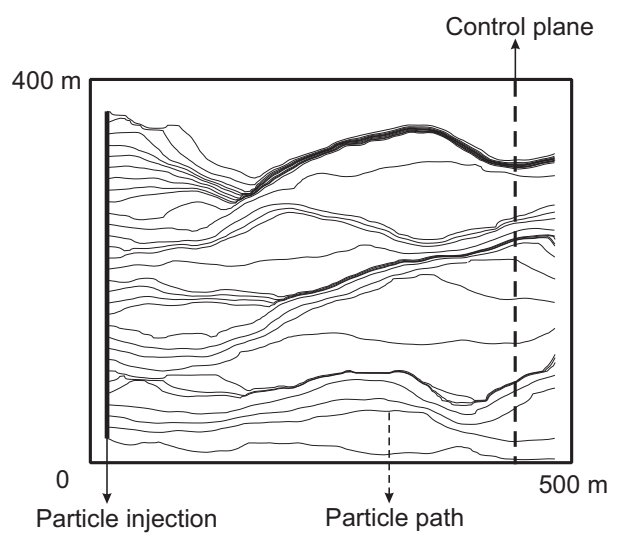

Figure 3.5: Configuration of the transport prediction experiment showing the line source where particles are injected and the control plane where breakthrough curves are measured (a few particle paths are shown for illustration).

\section{Characterization of hydraulic conductivity}

Regarding the characterization of hydraulic conductivities, we compare the initial (unconditional) ensemble of realizations and the final ensembles once the updating (conditioning) process is completed, focusing on: (i) the two performance measures defined earlier, $A A E(\ln K)$ and $A A D(\ln K)$, (ii) individual realizations from the ensembles, (iii) ensemble averages, (iv) log-conductivity histograms, (v) probability maps of the two facies, and (vi) connectivity in the horizontal direction.

Table 3.3 lists the values of $A A E(\ln K)$ and $A A D(\ln K)$; these values show, quantitatively, that conditioning to piezometric heads by either the standard EnKF or the NS-EnKF improves the characterization of the spatial distribution of hydraulic conductivities. A reduction on the $A A E$ indicates a higher precision, while a reduction on the $A A D$ implies a smaller bias. These values also show that the NS-EnKF performs better than the standard EnKF.

Figure 3.6 shows a randomly selected realization and the ensemble mean after conditioning to piezometric heads for the two filtering approaches; the reference field is also shown. We notice that the continuity of the sand channels in the NS-EnKF case is better reproduced than for the standard EnKF both for the single realization and for the ensemble mean. In any case, it is quite remarkable how well the main patterns of continuity on the reference field are captured by the ensemble Kalman filters.

A more pronounced difference between the final results obtained by the two filters is observed when analyzing the log-conductivity histograms. Figure 3.7 


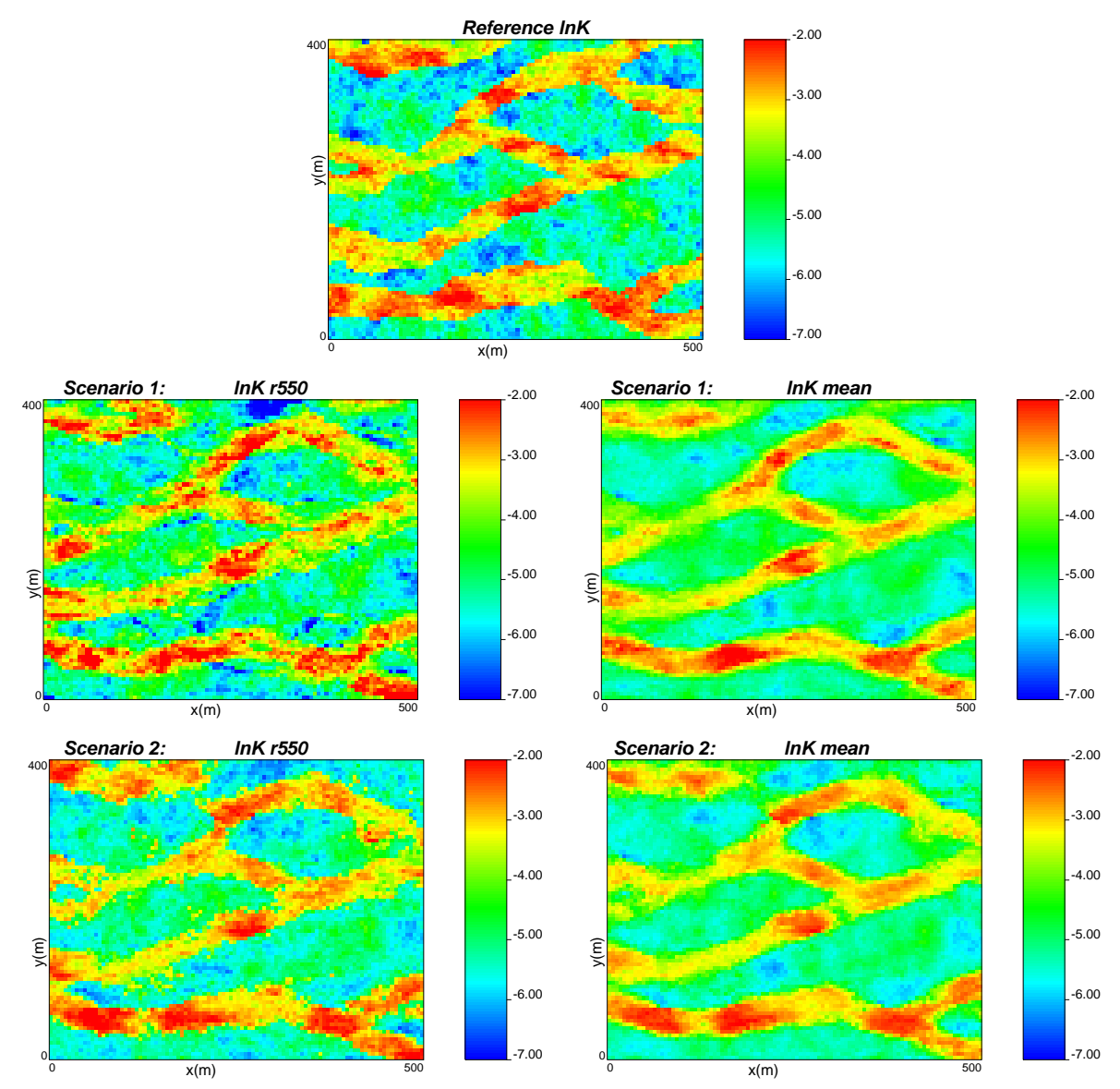

Figure 3.6: The reference field together with a randomly selected realization $\left(550^{\text {th }}\right)$ of $\ln K$ and the ensemble mean of $\ln K$ for Scenarios 1 and 2 (standard EnKF and NS-EnKF) after 50 times steps of piezometric head data assimilation. 
Table 3.3: Comparison of the initial ensemble (only conditioned to conductivity data), standard EnKF and NS-EnKF (Scenarios 1 and 2 in Table 3.1, which are conditioned to both conductivity and piezometric head data).

\begin{tabular}{lccc}
\hline Metrics & Initial ensemble & Scenario 1 & Scenario 2 \\
\hline $\operatorname{AAE}(\ln K)$ & 0.78 & 0.65 & 0.64 \\
$\operatorname{AAD}(\ln K)$ & 0.75 & 0.52 & 0.40 \\
$\operatorname{AAE}(\mathrm{h})$ & 0.48 & 0.12 & 0.13 \\
\hline $\operatorname{AAE}\left(T_{5 \%}\right)$ & 11.49 & 10.76 & 7.80 \\
$\operatorname{AAE}\left(T_{50 \%}\right)$ & 60.26 & 42.79 & 27.91 \\
$\operatorname{AAE}\left(T_{95 \%}\right)$ & 332.29 & 164.16 & 94.41 \\
$\operatorname{AAD}\left(T_{5 \%}\right)$ & 9.50 & 10.63 & 3.91 \\
$\operatorname{AAD}\left(T_{50 \%}\right)$ & 60.01 & 34.80 & 27.90 \\
$\operatorname{AAD}\left(T_{95 \%}\right)$ & 220.37 & 134.39 & 79.39 \\
\hline
\end{tabular}

displays the histogram of the initial ensemble of log-conductivity (recall that both filters start with the same ensemble of initial realizations conditioned to the hard log-conductivity data) and the histograms of the final ensembles. The initial ensemble pattern is maintained by the NS-EnKF as expected, since, this is a built-in feature of the new algorithm. However, the standard EnKF produces a histogram that gets close to Gaussian: the two initial modes are smeared out and the final range is increased.

Another way to look at how the evolution of the histograms impacts the characterization of the spatial distribution of the hydraulic conductivities is to retrieve the facies distribution by using the value of $-4 \ln (\mathrm{cm} / \mathrm{s})$ as a cutoff to identify the sand and clay facies. By transforming all realizations into 0 's (if $\ln K$ is less than -4 ) and 1 's (if $\ln K$ is greater than -4 ), and then computing the ensemble average, the resulting average maps would represent the probability that at each location the log-conductivity is larger than -4 , which, in this case, could be interpreted as that the facies is sand. Figure 3.8 shows the probability maps obtained in the initial ensemble, and on the final ensembles. In the initial ensemble, the hard log-conductivity data are enough to allow distinguish the location of the sand channels, but with still high uncertainty on their width. In the final ensembles, all channels are better delimited, with a very small area with probabilities away from 0 or 1 in the results of the NS-EnKF as compared with the probabilities obtained by the standard EnKF.

In a fluvial aquifer the connectivity induced by the sand channels is quite important in terms of transport predictions. Figure 3.9 shows connectivity 

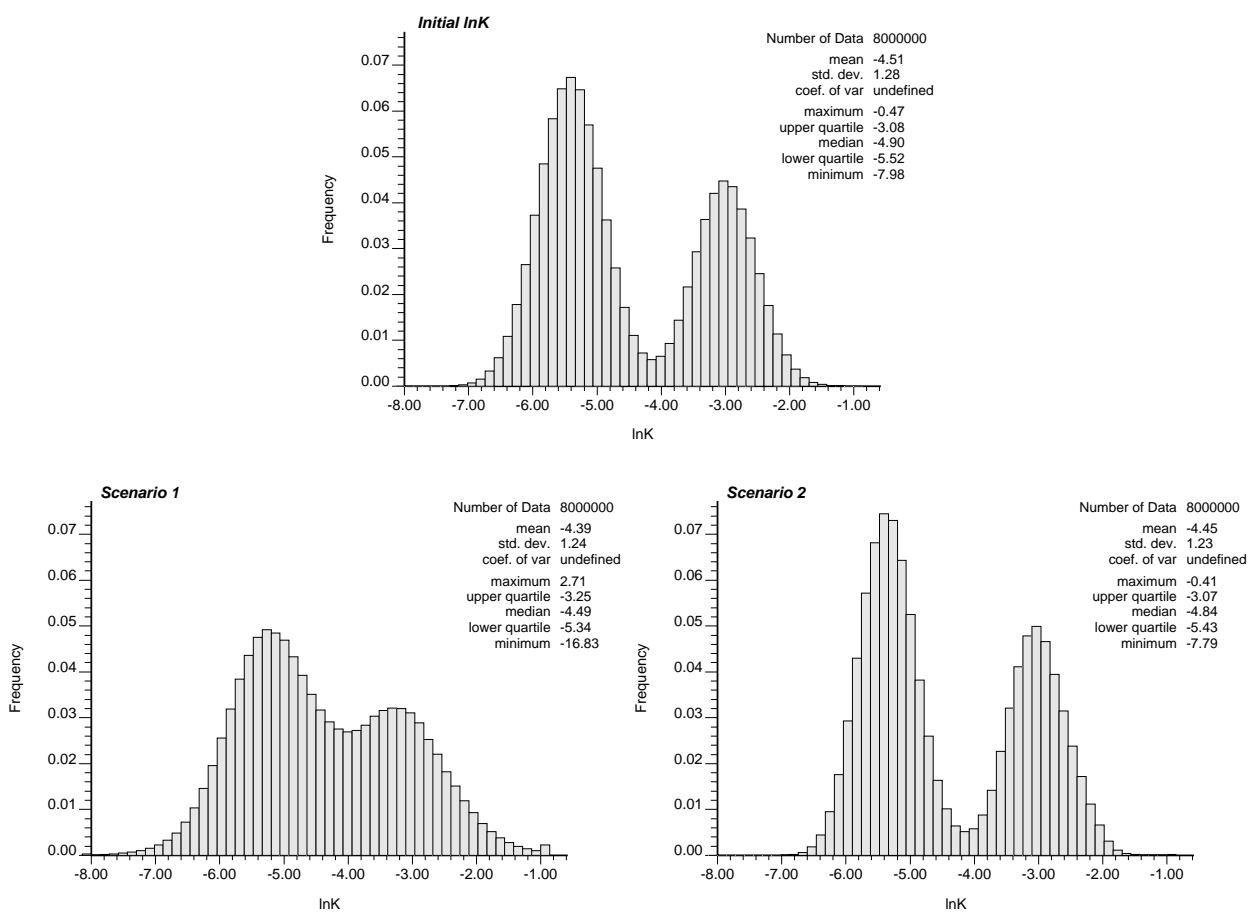

Figure 3.7: Log-conductivity histograms for the initial ensemble of realizations and for Scenarios 1 and 2 (standard EnKF and NS-EnKF) after data assimilation. 

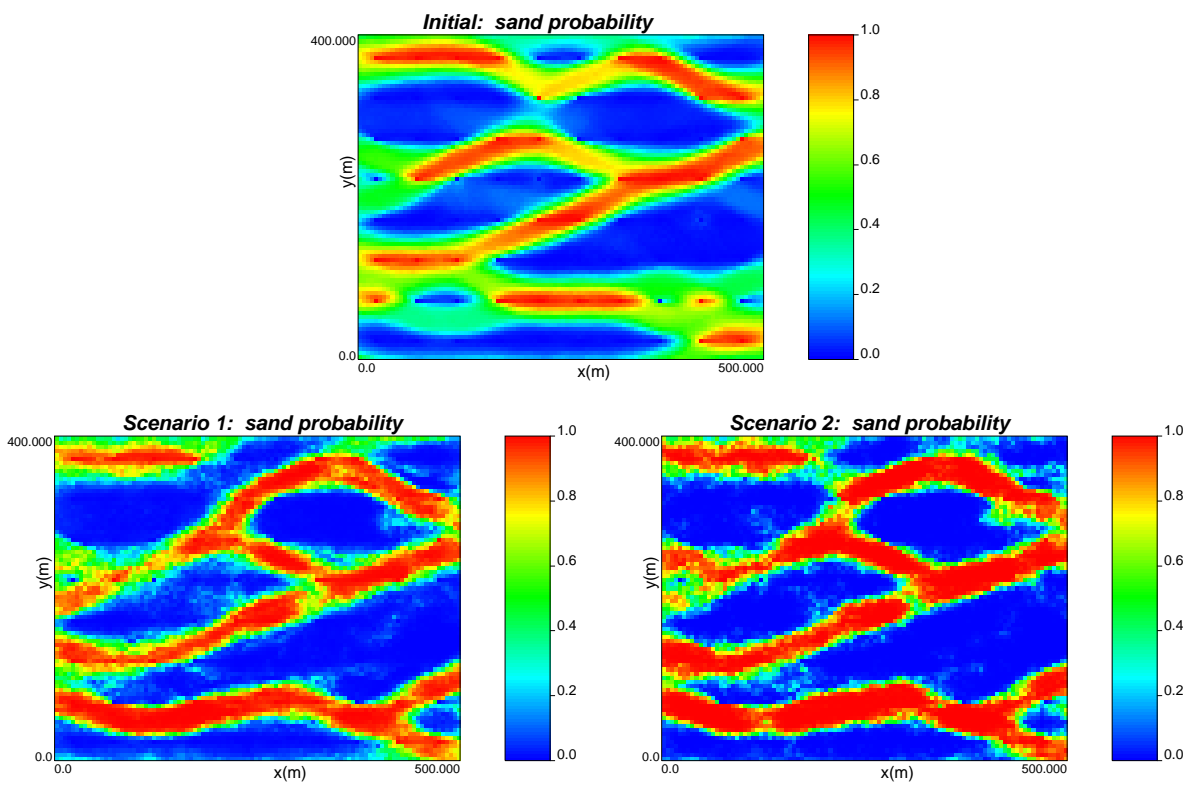

Figure 3.8: Probability that log-conductivity is larger than $-4 \ln (\mathrm{cm} / \mathrm{s})$ for the initial ensemble of realizations, and for the ensembles corresponding to Scenarios 1 and 2 (standard EnKF and NS-EnKF) after 50 times steps of piezometric head data assimilation. 
as a function of horizontal separation, for each one of the realizations in the initial ensemble and in the two final ensembles. Also the connectivity of the ensemble averages and of the reference field are shown. Connectivity is computed on the indicator maps obtained above and measures the probability that any two points, both of them with log-conductivities above $-4 \ln (\mathrm{cm} / \mathrm{s})$, and which are a certain horizontal distance apart, are connected by a continuos path of $\log$-conductivities above $-4 \ln (\mathrm{cm} / \mathrm{s})$. Looking only at the ensemble averages, we can conclude that the standard EnKF tends to produce realizations which are, on average, less connected, for all distances, than the reference field, with the NS-EnKF producing an average connectivity which is closer to the reference one than the standard EnKF; at the same time, the high connectivity values observed in the initial ensemble average for short distances is lost after conditioning to piezometric heads by any of the filters. Looking at the individual realizations, we can notice that the spread of the curves is progressively reduced from the initial ensemble to the ensemble obtained by the standard EnKF with the minimum spread for the ensemble obtained by the NS-EnKF. It remains to analyze the apparent splitting of the NS-EnKF ensemble of connectivity curves into two subsets, one set with curves about that of the reference field, and another one with curves higher than the reference.

In all the aspects analyzed, the NS-EnKF outperforms the standard EnKF with respect to the characterization of the spatial variability of log-conductivities.

\section{Prediction ability of the updated model}

Regarding the prediction capabilities of the updated models, we will analyze piezometric head predictions at both conditioning piezometers and control piezometers, we will also analyze the solute predictions for the mass transport experiment described earlier.

Figure 3.10 shows the hydraulic head evolution with time at two of the observation wells. Results are shown for the initial ensemble of realizations (conditioned only on log-conductivity) and for Scenarios 1 and 2 (standard EnKF and NS-EnKF) after conditioning to 32 piezometers up to the $50^{\text {th }}$ time step (22.8 days). Head uncertainty is significantly reduced after data assimilation. Conditioned piezometric heads, up to the $50^{\text {th }}$ time step, are almost perfectly reproduced in both scenarios. However, piezometric head prediction after the $50^{\text {th }}$ time step (when observation data are not used for conditioning anymore) exhibits lower uncertainty for the NS-EnKF than for the standard EnKF. We have also checked the head evolution at 8 control piezometers, two of which are shown in Figure 3.11. Like at the observation locations, piezometric head uncertainty is reduced, after data assimilation, in both scenarios, and the NS-EnKF gives more precise predictions than the standard EnKF. 

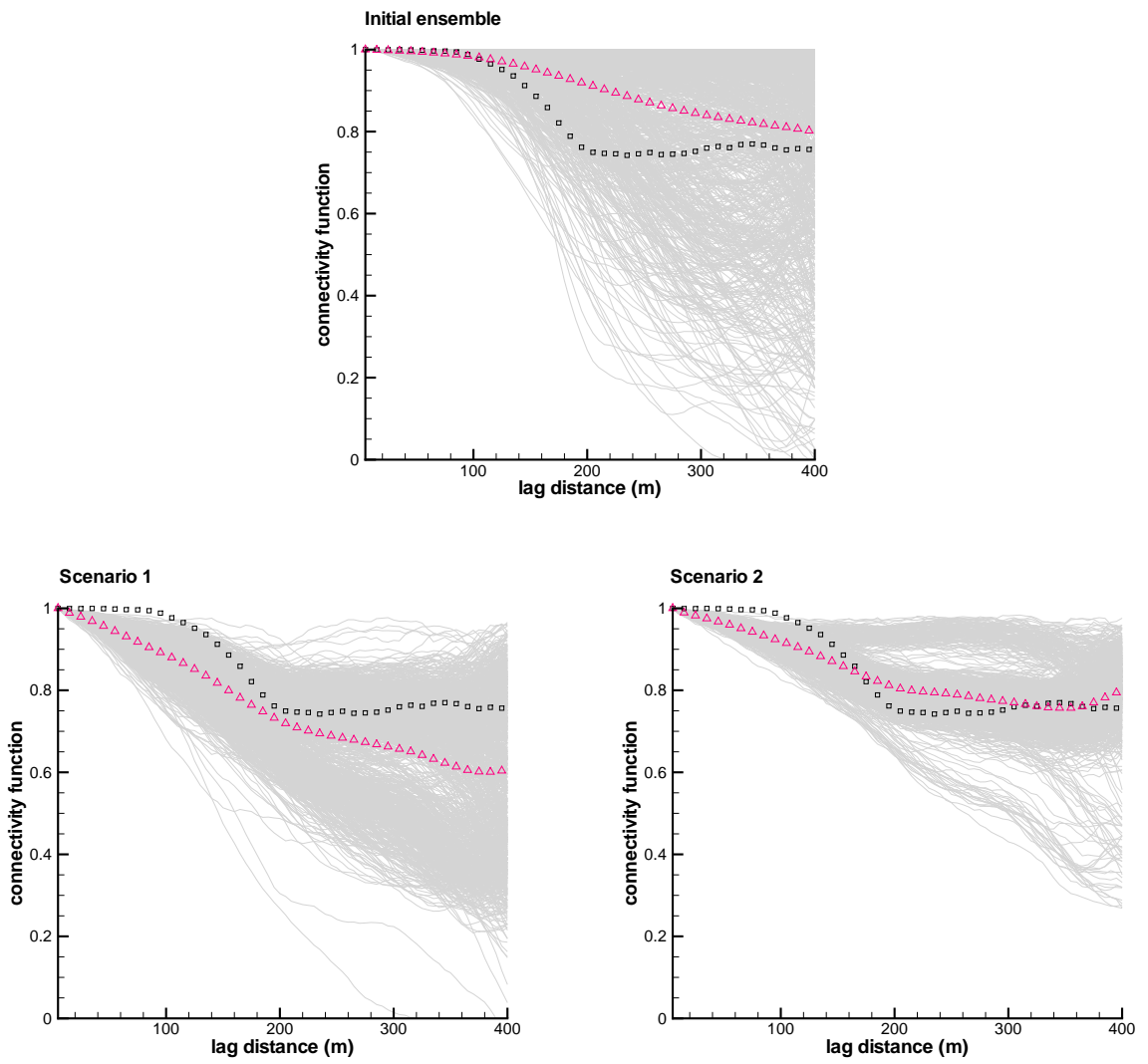

Figure 3.9: Connectivity, as a function of the separation distance, measuring the probability that two points aligned in the $x$ direction are connected by a continuous path of log-conductivities larger than $-4 \ln (\mathrm{cm} / \mathrm{s})$. Gray curves correspond to individual realizations, their mean is given by the triangles (red) and the squares (black) correspond to the reference. Results are shown for the initial ensemble of realizations (conditioned on log-conductivity) and for Scenarios 1 and 2 (standard EnKF and NS-EnKF) after data assimilation. 

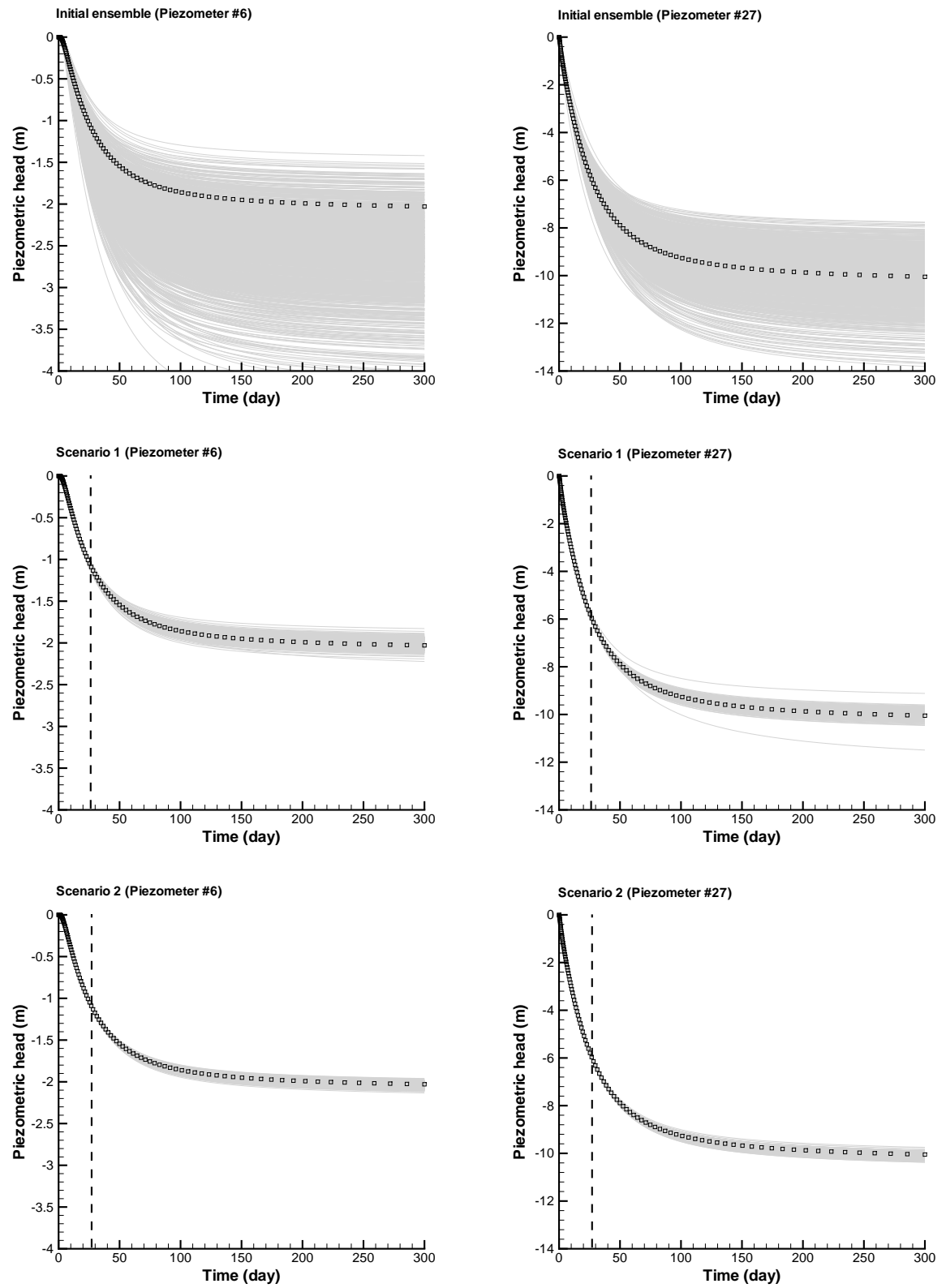

Figure 3.10: Piezometric head evolution at two of the thirty-two conditioning piezometers. Results are shown for the initial ensemble of realizations (conditioned only on log-conductivity) and for Scenarios 1 and 2 (standard EnKF and NS-EnKF, conditioned to transient piezometric heads up to 22.8 days). The vertical dashed lines indicate the conditioning period. Squares represent the piezometric head in the reference field. 

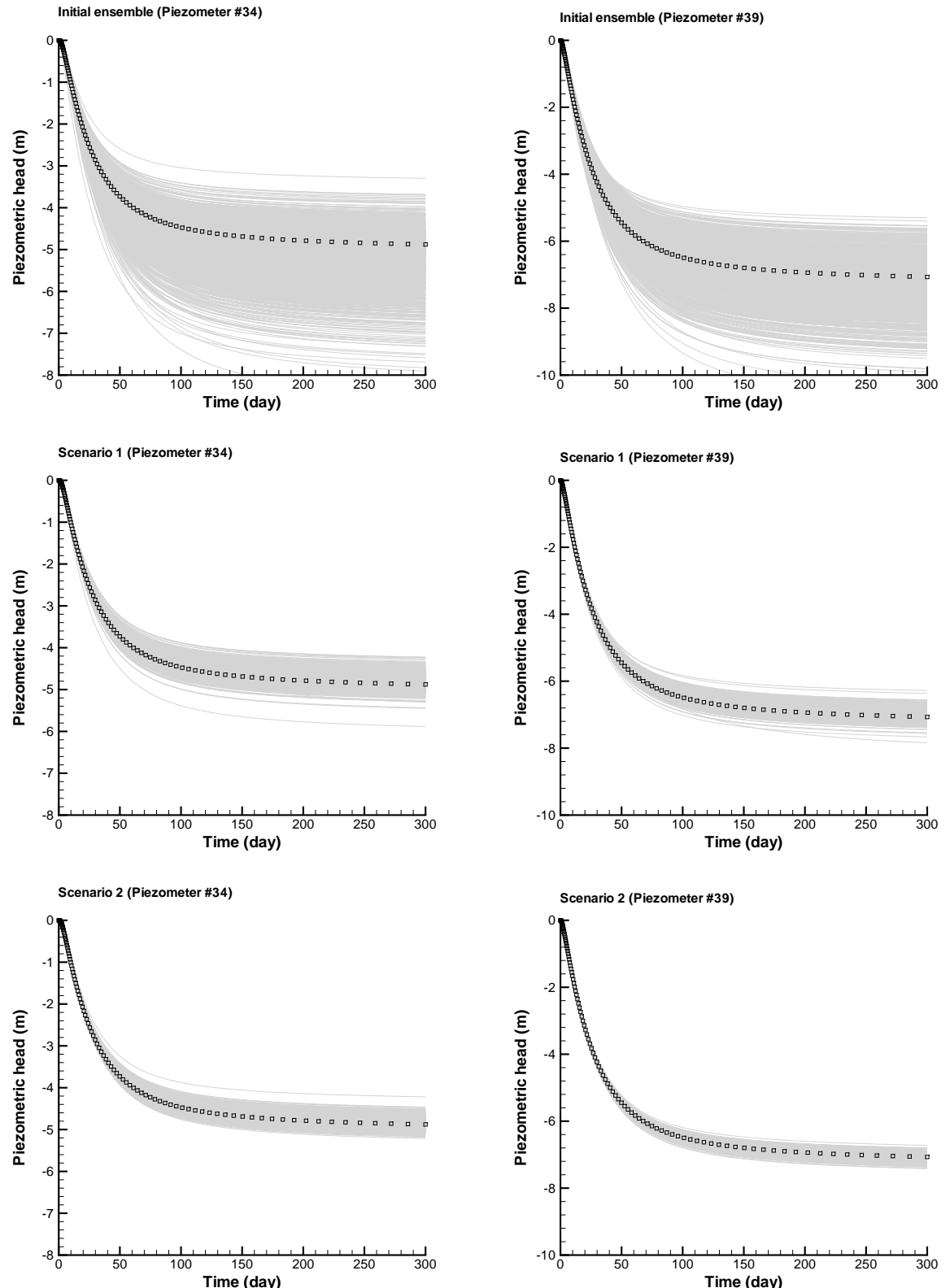

Figure 3.11: Piezometric head evolution at two of the eight validation piezometers. Results are shown for the initial ensemble of realizations (conditioned only on log-conductivity) and for Scenarios 1 and 2 (standard EnKF and NSEnKF) after data assimilation. Squares represent the piezometric head in the reference field. 
The updated $\ln K$ fields are further evaluated by using them as input for the solute transport prediction exercise described at the end of Section 3.4. The random walk particle tracking program RW3D (Fernàndez-Garcia et al., 2005; Salamon et al., 2006; Li et al., 2011a) is used to solve Equation 3.2. For the sake of simplicity, only advection is considered in the experiment. Table 3.3 shows the values of $A A E\left(T_{\alpha}\right)$ and $A A D\left(T_{\alpha}\right)$ for the $5^{\text {th }}, 50^{\text {th }}$ and $95^{\text {th }}$ percentiles of the breakthrough curves. We can see that, when using the NSEnKF, both the bias and uncertainty are substantially reduced with respect to the corresponding values computed on the initial ensemble. The standard EnKF also improves bias and uncertainty compared with the initial ensemble, although this improvement is less than that of the NS-EnKF. Figure 3.12 summarizes the breakthrough curves for the initial ensemble of realizations (conditioned only on log-conductivity) and for Scenarios 1 and 2 (standard EnKF and NS-EnKF) after data assimilation. The NS-EnKF gives better transport prediction than the standard EnKF, the breakthrough curve uncertainty, as measured by the width of the confidence interval given by the $5^{\text {th }}$ and $95^{\text {th }}$ percentiles, is reduced significantly, and the median BTC is almost identical to the reference one.

In summary, with respect to prediction of flow and transport, the standard EnKF is able to predict head evolution properly, and makes a reasonable prediction of solute transport, but cannot match the NS-EnKF, which performs better in both cases.

\subsubsection{Parameter sensitivity on NS-EnKF}

From the previous section we can conclude that the proposed approach, the NS-EnKF, is better than the standard EnKF. Next, we will analyze the sensitivity of the NS-EnKF to different parameters, i.e., time span of data assimilation, number of realizations, number of conditioning hard data and logconductivity variance.

\section{Effect of time span of head data assimilation}

In this section we will discuss the impact of the duration of the piezometric head data assimilation period on the spatial characterization of log-conductivity. Figures 3.13 and 3.14 show the ensemble mean and standard deviation of logconductivity for the initial ensemble and after assimilating head data up to time steps 1,10 and 50 (that is, $0.11,1.45$ and 22.8 days).

From the analysis of these two figures, we note that just a single time step is needed to obtain an ensemble mean that displays channels similar to those in the reference field. Then, as more observation heads are assimilated, the ensemble mean tends to resemble better the reference; however, the ensemble 

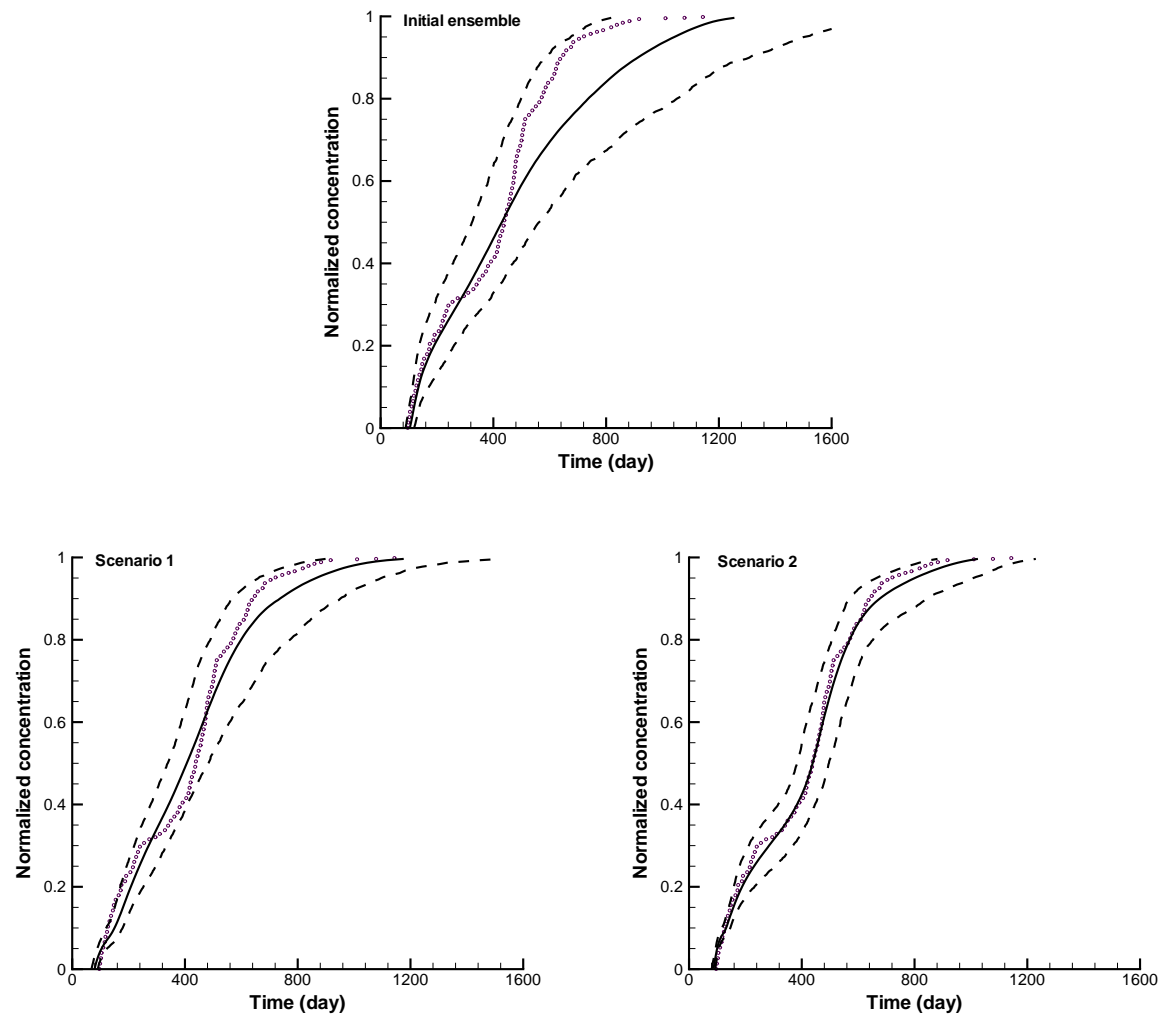

Figure 3.12: Summary of the breakthrough curves. The $5^{\text {th }}$ percentile, the median, and the $95^{\text {th }}$ percentile of the travel times are computed as a function of normalized concentration. Dashed lines correspond to the $5^{\text {th }}$ and $95^{\text {th }}$ percentiles, the solid line corresponds to the median, and the dotted line is the breakthrough curve in the reference. Results are shown for the initial ensemble of realizations (conditioned only on log-conductivity) and for Scenarios 1 and 2 (standard EnKF and NS-EnKF) after data assimilation. 

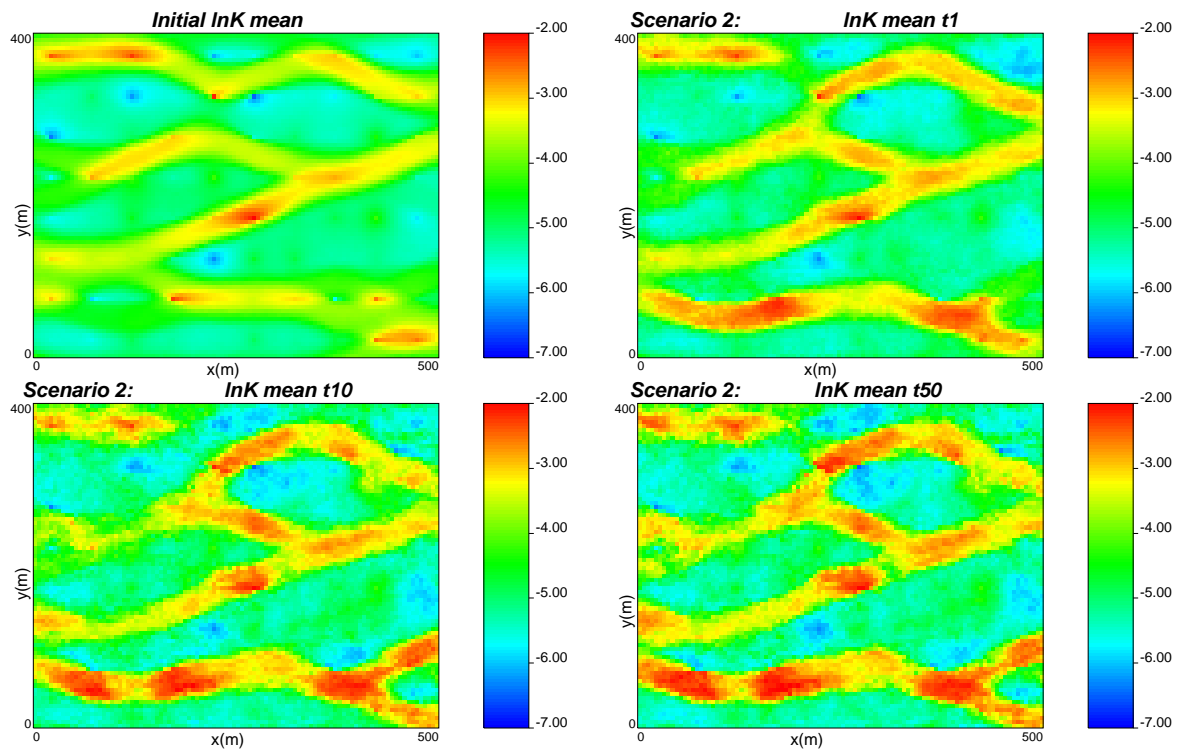

Figure 3.13: Evolution of log-conductivity ensemble mean as data are assimilated for time steps 1, 10 and 50 (Scenario 2).
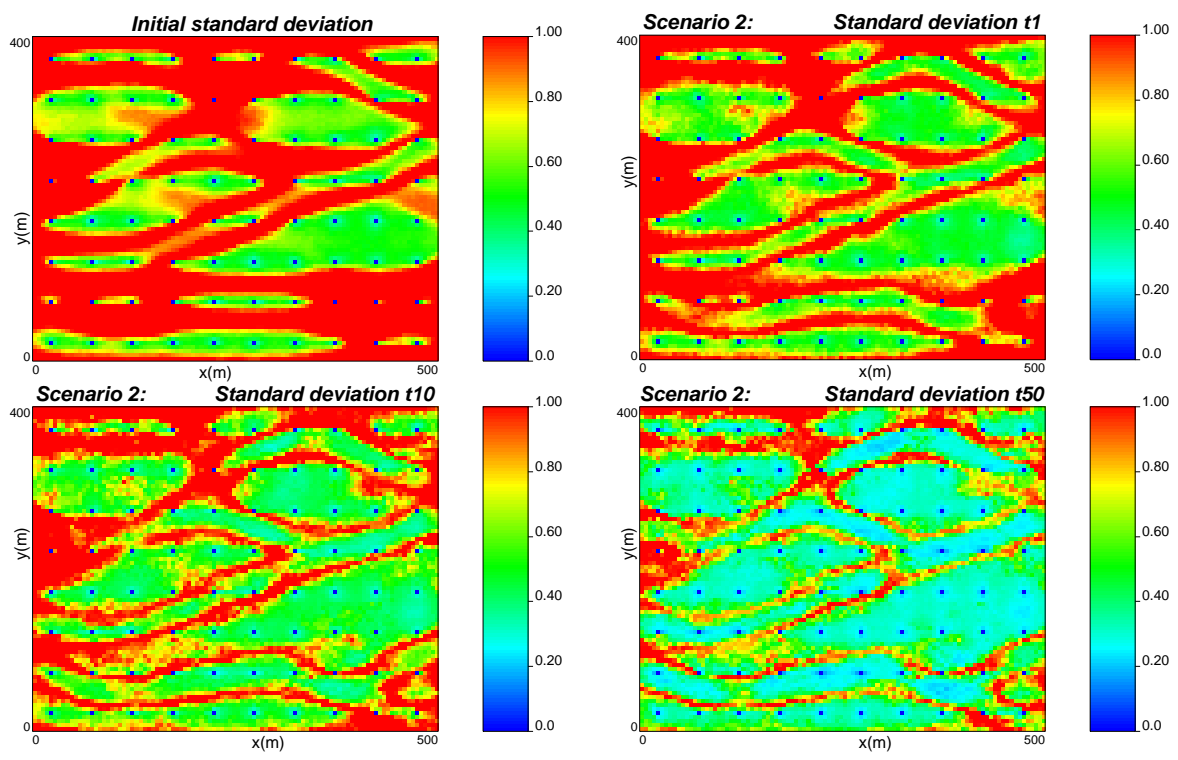

Figure 3.14: Evolution of log-conductivity ensemble standard deviation as data are assimilated for time steps 1, 10 and 50 (Scenario 2). 
mean does not change as much between the $10^{\text {th }}$ and the $50^{\text {th }}$ time steps as it did between the $1^{\text {st }}$ and the $10^{\text {th }}$.

The length of the assimilation period has a larger impact in the ensemble standard deviation maps. These maps show how the uncertainty on logconductivity becomes smaller as the time span of head data assimilation increases. As mentioned above, the ensemble mean maps change little between steps $10^{\text {th }}$ and $50^{\text {th }}$, but the standard deviation maps do change substantially, with an overall reduction of its values everywhere and with the highlight of the most likely locations for the channel borders given by the highest standard deviation values. In these maps, we can also observe that the standard deviation is zero at log-conductivity conditioning data locations, implying that $\ln K$ measurements are honored in all realizations.

We conclude that, for the specific flow setup discussed in this paper, a short span of piezometric head data is enough to obtain an ensemble mean capturing the main patterns of variability of log-conductivity, both in terms of facies delineation and of within-facies log-conductivity spatial variability; however, the ensemble of final realizations gets more precise when a longer time span is used for conditioning, resulting in an ensemble standard deviation map that could be used to draw the borders of the sand/clay interface.

\section{Effect of size of the ensemble}

All results shown until now were for an ensemble of 1000 realizations. To evaluate the impact of ensemble size, we performed an analysis with an ensemble of 200 realizations, referred to as Scenario 3 in Table 3.1. The ensemble log-conductivity mean, together with a few realizations before and after assimilating the piezometric head data for the first 50 time steps are displayed in Figure 3.15. For this number of realizations, and for our example, which has a relatively high log-conductivity variance, the problem of filter inbreeding (Hendricks Franssen and Kinzelbach, 2008) is apparent. The final updated realizations are almost identical to each other, and virtually equal to the ensemble mean after data assimilation. The average absolute deviation $A A D(\ln K)$ is only 0.02 (see Table 3.4 , Scenario 3 ), which represents an unreasonable reduction of uncertainty. A series of algorithms have been proposed to cope with the problem of filter degeneration caused by a small ensemble size (Chen and Oliver, 2010; Hamill et al., 2001; Hendricks Franssen and Kinzelbach, 2008; Houtekamer and Mitchell, 1998). We have not investigated how these algorithms would perform in conjunction with the NS-EnKF, and we conclude that the ensemble size has to be carefully chosen, particularly for high-variance log-conductivity fields. 

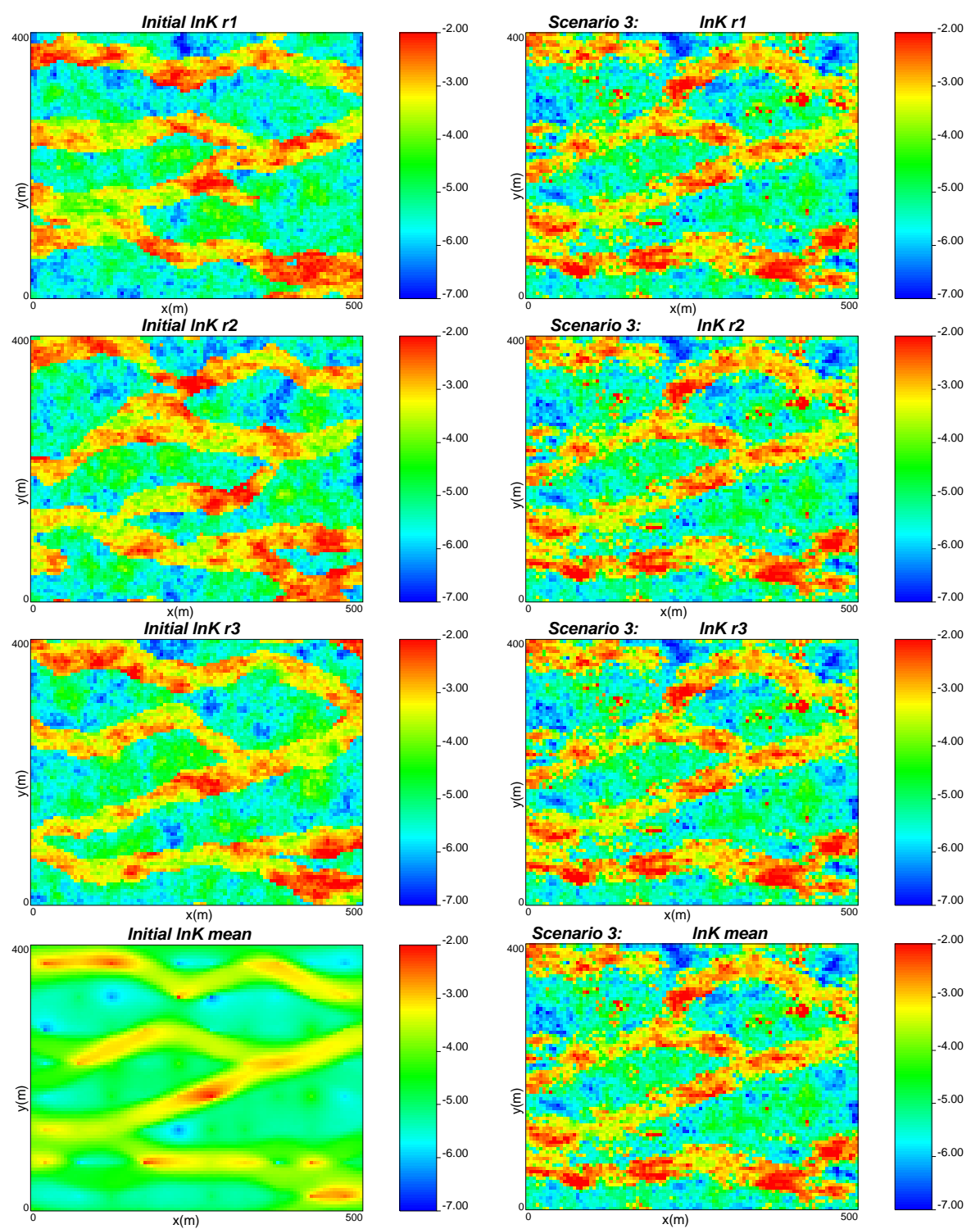

Figure 3.15: The first three realizations and the ensemble mean before (left column) and after (right column) 50 times steps of piezometric head data assimilation using the NS-EnKF with an ensemble size of 200 realizations (Scenario 3). Notice how filter inbreeding effects the final results making all the realizations virtually the same. 
Table 3.4: Performance measures for Scenarios 3, 4 and 5.

\begin{tabular}{lllllll}
\hline Metrics & $\begin{array}{l}\text { Initial } \\
\text { ensem- } \\
\text { ble }\end{array}$ & $\begin{array}{l}\text { Scenario } \\
3\end{array}$ & $\begin{array}{l}\text { Initial } \\
\text { ensem- } \\
\text { ble }\end{array}$ & $\begin{array}{l}\text { Scenario } \\
4\end{array}$ & $\begin{array}{l}\text { Initial } \\
\text { ensem- } \\
\text { ble }\end{array}$ & $\begin{array}{l}\text { Scenario } \\
5\end{array}$ \\
\hline $\operatorname{AAE}(\ln K)$ & 0.78 & 0.85 & 1.05 & 0.77 & 1.87 & 1.57 \\
$\operatorname{AAD}(\ln K)$ & 0.76 & 0.02 & 1.00 & 0.53 & 1.81 & 1.00 \\
$\operatorname{AAE}(\mathrm{h})$ & 0.52 & 0.52 & 1.34 & 0.14 & 0.77 & 0.43 \\
\hline $\operatorname{AAE}\left(T_{5 \%}\right)$ & - & - & 12.01 & 8.54 & 9.59 & 10.77 \\
$\operatorname{AAE}\left(T_{50 \%}\right)$ & - & - & 79.66 & 91.09 & 1986.06 & 1345.58 \\
$\operatorname{AAE}\left(T_{95 \%}\right)$ & - & - & 176.32 & 71.45 & 19313.82 & 4664.59 \\
$\operatorname{AAD}\left(T_{5 \%}\right)$ & - & - & 12.10 & 7.70 & 9.51 & 3.68 \\
$\operatorname{AAD}\left(T_{50 \%}\right)$ & - & - & 70.44 & 32.67 & 1668.17 & 831.16 \\
$\operatorname{AAD}\left(T_{95 \%}\right)$ & - & - & 158.70 & 71.20 & 13698.18 & 3134.55 \\
\hline
\end{tabular}

\section{Effect of number of hard conditioning data}

Maintaining an ensemble size of 1000 realizations, we reduce the number of $\ln K$ conductivity conditioning hard data. It could be argued that the good results observed in the previous section were due to the large number of conditioning data. This large number yields an initial ensemble average that already captures the main patterns of the reference $\ln K$ variability. For this reason we have reduced the number of conditioning data to 20 regularly-spaced samples taken from the reference field (Scenario 4 in Table 3.1). Then, we have generated a new set of initial $\ln K$ realizations by direct sampling using the same training image of Figure 3.2. The ensemble average of this new set of initial realizations can be seen in Figure 3.16. This mean map displays hardly any information about the channels of the reference, even though all 1000 realizations share the same 20 conditioning data.

It is remarkable to see how, assimilating the piezometric head data, the resulting ensemble mean map displays the most important features in the reference, although the channels are not as connected as in the reference. However, the relative improvement of this updated ensemble mean with respect to the initial one is very important. We attribute this behavior to the coupling of the EnKF and the normal-score transforms applied to all members of the state vector as described earlier.

Individual realizations, such as number $550^{\text {th }}$, resemble the main channel patterns of the reference; however, they are noisier than similar realizations generated with a larger number of $\ln K$ conditioning data, and, somehow, the channel connectivity is obscured. The metrics obtained for the flow and transport exercises are given in Table 3.4 (Scenario 4). We can see that all of 

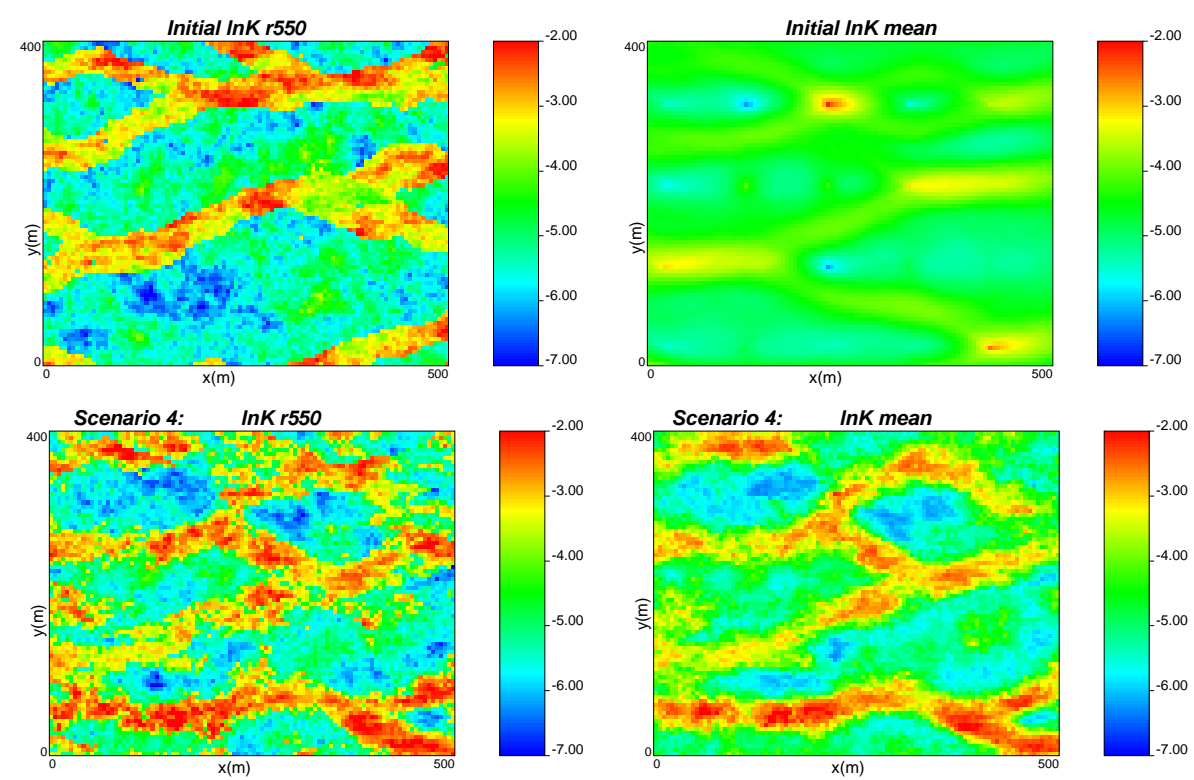

Figure 3.16: A randomly selected realization $\left(550^{\text {th }}\right)$ and the ensemble mean before and after 50 time steps of piezometric head data assimilation using the NS-EnKF for the scenario where only 20 log-conductivity conditioning data are used (Scenario 4). 
them decrease their values with respect to the values computed in the initial ensemble, and that the final values, although larger, are comparable to those obtained when conditioning to $80 \ln K$ data (Scenario 2 in Table 3.3).

We conclude that, overall, reducing the number of hard conditioning data is not an obstacle for log-conductivity characterization by the NS-EnKF.

\section{Effect of $\ln K$ variance}

Our final analysis focuses on the impact of a very high variance of $\ln K$ on the performance of the NS-EnKF (Scenario 5 in Table 3.1). To keep the rest of the parameters as similar as possible to those used in Scenario 2, we have simply scaled the reference realization, as well as the 1000 initial realizations for Scenario 2 , so that both reference and initial realizations have a variance of 9.92 , while keeping the same mean and the same spatial patterns. Then, we have run the same flow exercise in the new reference field, and we have sampled the same piezometers for their usage as conditioning data by the NS-EnKF. Figure 3.17 shows the reference field with its histogram, initial realization number 550 , the initial ensemble mean, the updated realization number 550 after assimilating the piezometric head data up to time step 50, and the updated ensemble $\ln K$ mean. We can see that even for this very large variance, the NS-EnKF is able to generate realizations which display the bimodal characteristic of the reference realization with, approximately, the same distribution of channels. We attribute the good behavior of the method to the normal-score transformation and to the large conductivity contrast that there is between sand and clay. The measures quantifying bias and uncertainty of log-conductivity characterization and flow and transport predictions are shown in Table 3.4 (Scenario 5). We can see that the biases and uncertainties are significantly reduced; however, we notice that the final bias and uncertainty values for the travel times after the NS-EnKF are still very high, which can be attributed to the extreme range of log-conductivities. This large range of variability induces zones of very low $K$ and of very high $K$ in all realizations; a small deviation of the proportions of the very low (or very high) log-conductivities with respect to the reference field can produce a large deviation on the travel times. The improvement of transport prediction is also well noticed when analyzing the BTCs (Figure 3.18).

We conclude that the NS-EnKF can handle high variance cases, particularly for bimodal cases with contrasting facies.

\subsubsection{Discussion}

We have demonstrated that the proposed NS-EnKF works on the fluvial aquifer used as a reference in this synthetic exercise. We have also shown 

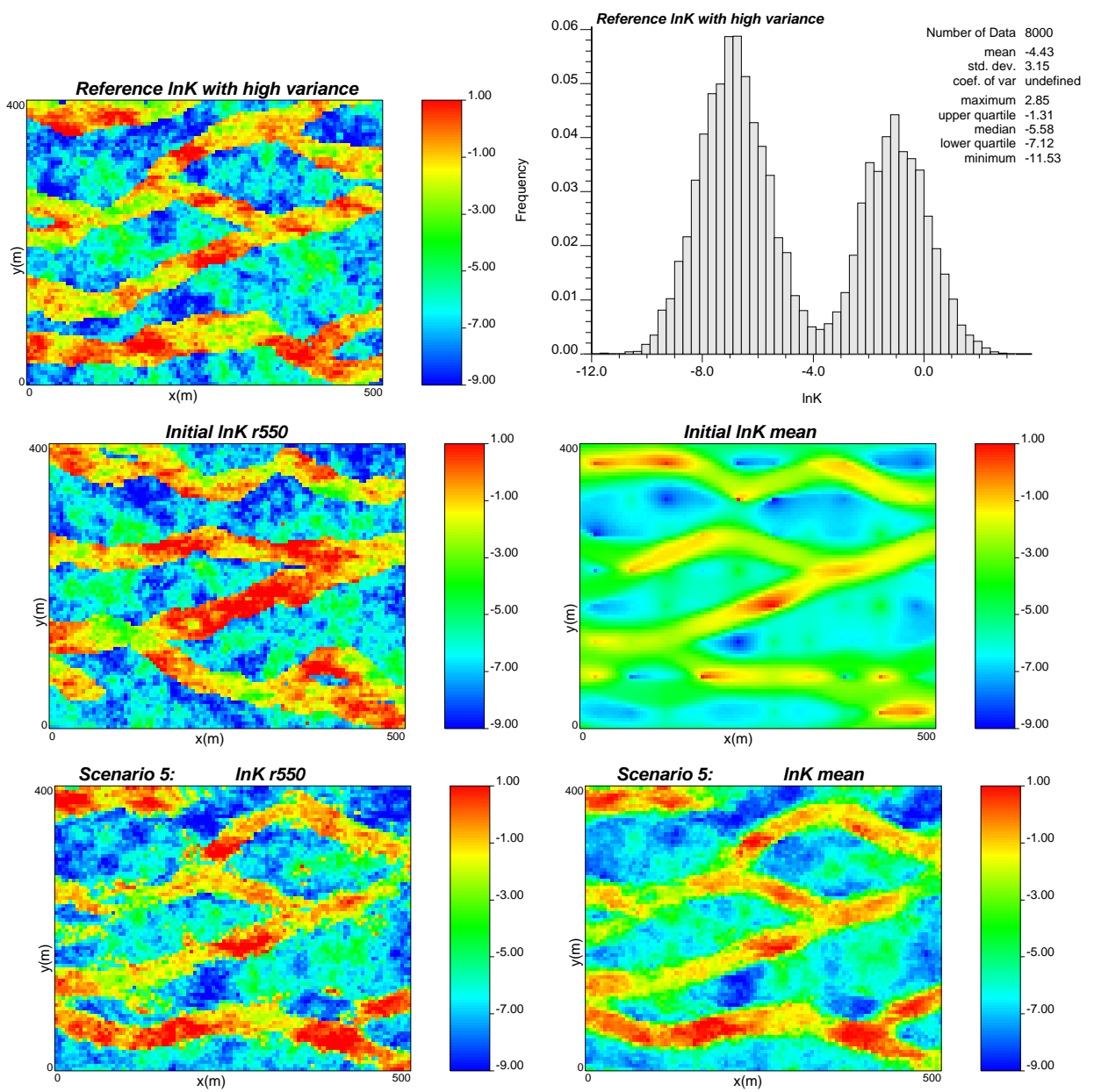

Figure 3.17: New reference log-conductivity field with larger variance (9.92 $\left.\ln (\mathrm{cm} / \mathrm{s})^{2}\right)$ and corresponding histogram, together with a realization $\left(550^{\text {th }}\right)$ and the ensemble mean before and after 50 times steps of piezometric head data assimilation using the NS-EnKF (Scenario 5). 

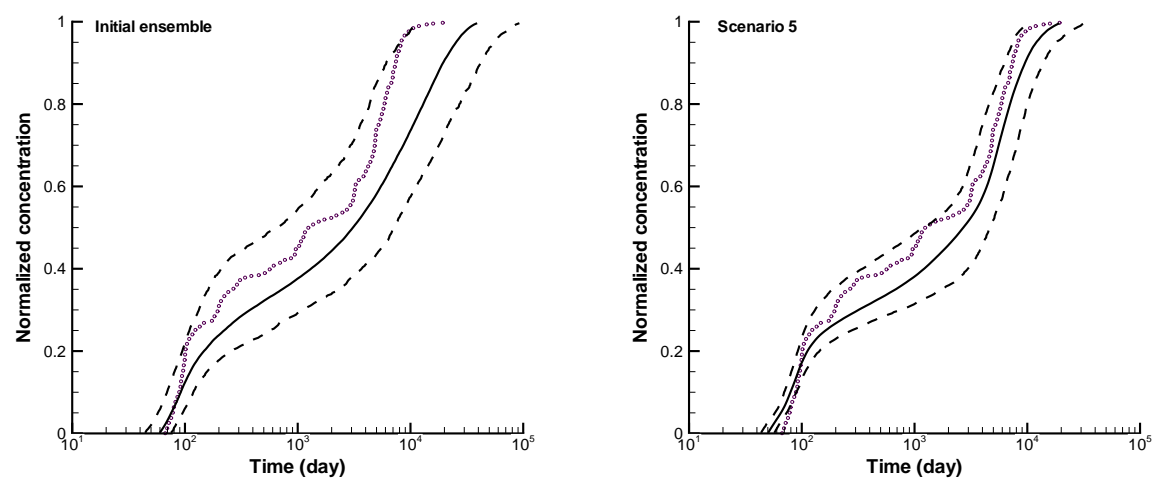

Figure 3.18: Summary of the breakthrough curves for Scenario 5. The $5^{\text {th }}$ percentile, the median, and the $95^{\text {th }}$ percentile of the travel times are computed as a function of normalized concentration. Results are shown for the initial ensemble of realizations (conditioned only on log-conductivity) and for the realizations after data assimilation.

that the method is quite robust for scenarios under conditions less favorable than the ones of the reference scenario (Scenario 2). The starting point is the less amenable for a Kalman filter: the state transition equation is a complex non-linear function, and both log-conductivities and piezometric heads can be statistically characterized by a multivariate distribution which is far from the multiGaussian one. Kalman filter performance is the best for linear state transition equations and for multiGaussian random functions. We have chosen to transform both parameters and state variables (all collected in a joint state vector) into new ones which follow Gaussian marginal distributions. This transformation does not imply that the bivariate, trivariate and higher-order distributions of the transformed random function are multiGaussian. We have also chosen the ensemble Kalman filter to condition to piezometric head data, because, out of the different Kalman filters, it is the most robust against nonlinearities of the state transition function.

The main conclusion that we derive from this work is that, in a similar way to the EnKF is robust to non-linearities; the marginal transformation into Gaussian deviates of conductivities and piezometric heads is robust to non-multiGaussianity. It could be argued that the results are so good because the number of hard conditioning data is large. There are two replies to this argument: (i) the number of hard conditioning data is the same for the standard EnKF as for the NS-EnKF in the examples, yet NS-EnKF always performs better, and (ii) one of the scenarios analyzed reducing substantially 
the number of conditioning data yields similar results. In addition, we have also performed an analysis with no hard data, which is included in a paper currently under review (Zhou et al., 2011), and the results are remarkably good; not as good as we present here, but good enough to warrant that the benefits of the NS-EnKF are worth considering.

\subsection{Conclusions}

The EnKF has gained popularity in diverse disciplines because of its computational efficiency, flexibility and capacity for uncertainty assessment. However, although the EnKF is relatively robust for nonlinear model dynamics, it performs not optimally for non-multiGaussian parameter distributions. In this work, a new approach (NS-EnKF) is proposed that always works on state variables with marginal Gaussian distributions. Gaussianity is achieved by applying a normal-score transform to all variables, prior to performing the updating step in Kalman filtering. We recognize that the normal-score transform of the state variables renders them just univariate Gaussian, and that the multivariate distribution may remain far from the optimal multiGaussian distribution; yet, the new state variables are closer to multiGaussian than prior to the transformation, and, the histograms of the state variables (conductivity and piezometric head in our case) are preserved throughout the entire process. We have demonstrated how to implement the NS-EnKF to generate log-conductivity realizations which are conditional to log-conductivity data and also to transient piezometric head data. The method not only is able to honor the conditioning data, but it preserves the bimodal characteristics of the underlying reference field, performs well in the context of hydraulic head predictions outside the conditioning period, and also in the context of mass transport prediction. We have also analyzed the impact in the performance of the NS-EnKF of the following parameters: the length of the piezometric head sampling period, the number of conditioning log-conductivity data, the number of realizations in the ensemble and the variance of log-conductivity.

The following conclusions can be drawn from the simulation experiments:

- The proposed method, the NS-EnKF, gives better results than the standard EnKF in terms of reproducing the bimodal $\ln K$ histogram, the connectivity of the $\ln K$ fields and in terms of predicting hydraulic heads and conservative transport. Both methods are capable of generating $\ln K$ realizations which honor the conditioning data, namely log-conductivity and transient head data. On the contrary, the standard EnKF cannot preserve the bimodal histogram of $\ln K$, underestimates connectivity and gives less precise and less accurate transport predictions than the NS-EnKF for the transport exercise discussed. 
- The hydraulic head measurements during the early stage of the assimilation process by the NS-EnKF are enough to capture the main patterns of variability of $\ln K$; however, by extending the assimilation time span, the uncertainty on $\ln K$ is largely decreased, and the ensemble standard deviation map serves to delineate the interfaces between sand and clay.

- In this experiment good results were obtained with 1000 realizations, but results deteriorate for 200 realizations. Careful choice of the ensemble size is important in the application of the NS-EnKF.

- A large number of log-conductivity conditioning data helps in delineating the spatial variability patterns before the hydraulic head is assimilated by the NS-EnKF; however, we found that even when the number of hard data is smaller, piezometric head observations are enough to capture the complex patterns exhibited in this particular example.

- The NS-EnKF also performs well in strongly heterogeneous formations with $\ln K$ variance close to 10 .

The univariate Gaussian distribution is considered in the present method and bivariate or even multivariate Gaussian distribution will be investigated in the future. 


\section{Bibliography}

Alcolea, A., Carrera, J., Medina, A., 2006. Inversion of heterogeneous parabolic-type equations using the pilot points method. Int J Numer Meth Fl 51 (9-10), 963-980.

Arulampalam, M. S., Maskell, S., Gordon, N., Clapp, T., 2002. A tutorial on particle filters for online nonlinear/non-Gaussian Bayesian tracking. IEEE Transactions on signal processing 50 (2), 174-188.

Béal, D., Brasseur, P., Brankart, J. M., Ourmières, Y., Verron, J., 2010. Characterization of mixing errors in a coupled physical biogeochemical model of the North Atlantic: implications for nonlinear estimation using Gaussian anamorphosis. Ocean Science 6, 247-262.

Bear, J., 1972. Dynamics of fluids in porous media. American Elsevier Pub. Co., New York, 764pp.

Bertino, L., Evensen, G., Wackernagel, H., 2003. Sequential data assimilation techniques in oceanography. International Statistical Review 71 (2), 223241.

Bocquet, M., Pires, C. A., Wu, L., 2010. Beyond Gaussian statistical modeling in geophysical data assimilation. Monthly Weather Review 138 (8), 29973023.

Burgers, G., Jan van Leeuwen, P., Evensen, G., 1998. Analysis scheme in the ensemble Kalman filter. Monthly Weather Review 126 (6), 1719-1724.

Carrera, J., Alcolea, A., Medina, A., Hidalgo, J., Slooten, L. J., 2005. Inverse problem in hydrogeology. Hydrogeology Journal 13 (1), 206-222.

Chen, Y., Oliver, D. S., 2010. Cross-covariances and localization for EnKF in multiphase flow data assimilation. Computational Geosciences 14 (4), 579-601.

Chen, Y., Oliver, D. S., Zhang, D., 2009. Data assimilation for nonlinear problems by enemble Kalman filter with reparameterization. Journal of Petroleum Science and Engineering 66, 1-14. 
Chen, Y., Zhang, D., 2006. Data assimilation for transient flow in geologic formations via ensemble Kalman filter. Advances in Water Resources 29 (8), $1107-1122$.

De Marsily, G., Delhomme, J. P., Coudrain-Ribstein, A., Lavenue, A. M., 2000. Four decades of inverse problems in hydrogeology. In: Zhang, D., Winter, C. L. (Eds.), Theory, modeling, and field investigation in hydrogeology: a special volume in honor of Shlomo P. Neumans 60th birthday. Geological Society of America Special Paper 348, Boulder, Colorado, pp. $1-17$.

Deutsch, C., Tran, T., 2002. FLUVSIM: a program for object-based stochastic modeling of fluvial depositional systems. Computers \& Geosciences 28 (4), $525-535$.

Deutsch, C. V., Journel, A. G., 1998. GSLIB, Geostatistical Software Library and User's Guide, 2nd Edition. Oxford University Press, New York, 384pp.

Evensen, G., 1994. Sequential data assimilation with a nonlinear quasigeostrophic model using Monte Carlo methods to forecast error statistics. Journal of Geophysical Research 99 (C5), 10143-10162.

Evensen, G., 2003. The Ensemble Kalman Filter: Theoretical formulation and practical implementation. Ocean dynamics 53 (4), 343-367.

Evensen, G., 2007. Data assimilation: The ensemble Kalman filter. Springer Verlag, 279pp.

Fernàndez-Garcia, D., Illangasekare, T., Rajaram, H., 2005. Differences in the scale dependence of dispersivity and retardation factors estimated from forced-gradient and uniform flow tracer tests in three-dimensional physically and chemically heterogeneous porous media. Water Resources Research 41 (3), W03012.

Fu, J., 2008. A Markov chain Monte Carlo method for inverse stochastic modeling and uncertainty assessment. Ph.D. thesis, Technical University of Valencia. 136pp.

Gómez-Hernández, J. J., Journel, A. G., 1993. Joint sequential simulation of Multi-Gaussian fields. In: Soares, A. (Ed.), Geostatistics Tróia '92. Vol. 1. Kluwer Academic Publishers, Dordrecht, pp. 85-94.

Gómez-Hernández, J. J., Sahuquillo, A., Capilla, J. E., 1997. Stochastic simulation of transmissivity fields conditional to both transmissivity and piezometric data-I. Theory. Journal of Hydrology 203 (1-4), 162-174. 
Gómez-Hernández, J. J., Wen, X., 1994. Probabilistic assessment of travel times in groundwater modeling. J. of Stochastic Hydrology and Hydraulics 8 (1), 19-56.

Gómez-Hernández, J. J., Wen, X. H., 1998. To be or not to be multi-Gaussian? A reflection on stochastic hydrogeology. Advances in Water Resources 21 (1), $47-61$.

Gu, Y., Oliver, D., 2007. An iterative ensemble kalman filter for multiphase fluid flow data assimilation. SPE Journal 12 (4), 438-446.

Gu, Y., Oliver, D. S., 2006. The ensemble Kalman filter for continuous updating of reservoir simulaiton models. Journal of Energy Resources Technology $128,79-87$.

Hamill, T. M., Whitaker, J. S., Snyder, C., 2001. Distance-dependent filtering of background error covariance estimates in an ensemble Kalman filter. Monthly Weather Review 129 (11), 2776-2790.

Hendricks Franssen, H. J., Alcolea, A., Riva, M., Bakr, M., van der Wiel, N., Stauffer, F., Guadagnini, A., 2009. A comparison of seven methods for the inverse modelling of groundwater flow. Application to the characterisation of well catchments. Advances in Water Resources 32 (6), 851-872.

Hendricks Franssen, H. J., Kinzelbach, W., 2008. Real-time groundwater flow modeling with the Ensemble Kalman Filter: Joint estimation for states and parameters and the filter inbreeding problem. Water Resources Research 44, W09408.

Hendricks Franssen, H. J., Kinzelbach, W., 2009. Ensemble Kalman filtering versus sequential self-calibration for inverse modelling of dynamic groundwater flow systems. Journal of Hydrology 365 (3-4), 261-274.

Houtekamer, P. L., Mitchell, H. L., 1998. Data assimilation using an ensemble Kalman filter technique. Monthly Weather Review 126 (3), 796-811.

Houtekamer, P. L., Mitchell, H. L., 2001. A sequential ensemble Kalman filter for atmospheric data assimilation. Monthly Weather Review 129 (1), 123137.

Hu, L. Y., 2000. Gradual deformation and iterative calibration of Gaussianrelated stochastic models. Mathematical Geology 32 (1), 87-108.

Journel, A. G., Deutsch, C. V., 1993. Entropy and spatial disorder. Mathematical Geology 25 (3), 329-355. 
Kalman, R. E., 1960. A new approach to linear filtering and prediction problems. Journal of Basic Engineering 82, 35-45.

Knudby, C., Carrera, J., 2005. On the relationship between indicators of geostatistical, flow and transport connectivity. Advances in Water Resources 28 (4), 405-421.

Leng, C., Yeh, H., 2003. Aquifer parameter identification using the extended Kalman filter. Water Resources Research 39 (3), 1062.

Li, L., Zhou, H., Gómez-Hernández, J. J., 2011a. Transport upscaling using multi-rate mass transfer in three-dimensional highly heterogeneous porous media. Advances in Water Resources, doi:10.1016/j.advwatres.2011.01.001.

Li, L., Zhou, H., Hendricks Franssen, H.-J., Gómez-Hernández, J. J., 2011b. Modeling transient groundwater flow by coupling ensemble Kalman filtering and upscaling. Water Resources Research, under review.

Liu, G., Chen, Y., Zhang, D., 2008. Investigation of flow and transport processes at the MADE site using ensemble Kalman filter. Advances in Water Resources 31, 975-986.

Mariethoz, G., Renard, P., Straubhaar, J., 2010. The direct sampling method to perform multiple-point geostatistical simulaitons. Water Resources Research 46, W11536.

McLaughlin, D., Townley, L. R., 1996. A reassessment of the groundwater inverse problem. Water Resources Research 32 (5), 1131-1161.

Meier, P. M., Medina, A., Carrera, J., 2001. Geostatistical inversion of crosshole pumping tests for identifying prferential flow channels within a shear zone. Ground water 39 (1), 10-17.

Moradkhani, H., Sorooshian, S., Gupta, H. V., Houser, P. R., 2005. Dual state-parameter estimation of hydrological models using ensemble Kalman filter. Advances in Water Resources 28 (2), 135-147.

Nowak, W., 2009. Best unbiased ensemble linearization and the quasi-linear Kalman ensemble generator. Water Resour Res 45, W04431.

Oliver, D. S., Chen, Y., 2010. Recent progress on reservoir history matching: a review. Computational Geosciences 15 (1), 185-221.

Pardo-Igúzquiza, E., Dowd, P. A., 2003. CONNEC3D: a computer program for connectivity analysis of 3D random set models. Computers \& Geosciences $29,775-785$. 
RamaRao, B., LaVenue, A., De Marsily, G., Marietta, M., 1995. Pilot point methodology for automated calibration of an ensemble of conditionally simulated transmissivity fields 1 . Theory and computational experiments. Water Resources Research 31 (3), 475-493.

Salamon, P., Fernàndez-Garcia, D., Gómez-Hernández, J., 2006. A review and numerical assessment of the random walk particle tracking method. Journal of Contaminant Hydrology 87 (3-4), 277-305.

Schöniger, A., Nowak, W., Hendricks Franssen, H. J., 2011. Parameter estimation by ensemble Kalman filters with transformed data: approach and application to hydraulic tomography. Water Resources Research, under review.

Simon, E., Bertino, L., 2009. Application of the Gaussian anamorphosis to assimilation in a 3-D coupled physical-ecosystem model of the North Atlantic with the EnKF: a twin experiment. Ocean Science (5), 495-510.

Stauffer, D., Aharony, A., 1994. Introduction to percolation theory. Taylor and Francis, London. 181pp.

Sun, A. Y., Morris, A. P., Mohanty, S., 2009. Sequential updating of multimodal hydrogeologic parameter fields using localization and clustering techniques. Water Resources Research 45 (7), W07424.

Wen, X., Chen, W., 2005a. Real-time reservoir model updating using ensemble Kalman filter. SPE 92991.

Wen, X. H., Chen, W. H., 2005b. Some practical issues on real-time reservoir model updating using ensemble Kalman filter. In: International Petroleum Technology Conference. Qatar, pp. doi: 10.2523/11024-MS.

Yeh, H., Huang, Y., 2005. Parameter estimation for leaky aquifers using the extended Kalman filter, and considering model and data measurement uncertainties. Journal of Hydrology (302), 28-45.

Yeh, W. W., 1986. Review of parameter identification procedures in groundwater hydrology: The inverse problem. Water Resources Research 22 (2), $95-108$.

Zhou, H., Li, L., Gómez-Hernández, J. J., Hendricks Franssen, H.-J., 2011. Pattern identification in bimodal aquifer with normal-score ensemble kalman filter. Mathematical Geosciences, under review.

Zinn, B., Harvey, C., 2003. When good statistical models of aquifer heterogeneity go bad: A comparison of flow, dispersion, and mass transfer in 
connected and multivariate Gaussian hydraulic conductivity fields. Water Resources Research 39 (3), 1051. 
Mathematical Geosciences, under review.

\author{
4 \\ Pattern Recognition in a \\ Bimodal Aquifer using the \\ Normal-Score Ensemble \\ Kalman Filter
}

\begin{abstract}
The ensemble Kalman filter (EnKF) is now widely used in diverse disciplines to estimate model parameters and update model states by integrating observed data. EnKF is known to perform optimally only for multiGaussian distributed states and parameters. A new approach, the normal-score EnKF (NS-EnKF), has been recently proposed to handle complex aquifers with nonGaussian distributed parameters. In this work, we aim at investigating the capacity of NS-EnKF to identify patterns in the spatial distribution of the model parameters (hydraulic conductivities) by assimilating dynamic observations in the absence of direct measurements of the parameters themselves. In some situations, hydraulic conductivity measurements (hard data) may not be available, which requires the estimation of conductivities from indirect observations, such as piezometric heads. We show how the NS-EnKF is capable of retrieving the bimodal nature of a synthetic aquifer solely from piezometric head data. By comparison with a more standard implementation of EnKF,
\end{abstract}


the NS-EnKF gives better results with regard to histogram preservation, uncertainty assessment and transport predictions.

\subsection{Introduction}

The inverse problem in hydrogeology involves characterizing model parameters, mainly hydraulic conductivity, by integrating measurements of the state variables such as hydraulic head or concentration data. One such inverse method is the ensemble Kalman filter (EnKF). The EnKF was proposed by Evensen (1994) and further clarified by Burgers et al. (1998) as an extension to the Kalman filter for the cases in which the state equation is non-linear. It has gained popularity in many disciplines as an efficient data assimilation algorithm (e.g., Houtekamer and Mitchell, 2001; Bertino et al., 2003), and it has been extended, through the augmentation of the state vector, to the identification of the parameters controlling the state of the system (Nævdal et al., 2005; Chen and Zhang, 2006; Moradkhani et al., 2005; Wen and Chen, 2005; Hendricks Franssen and Kinzelbach, 2008). The popularity of EnKF can be attributed to its advantages like the relative limited CPU demand as compared with other Monte-Carlo type inverse modeling (Hendricks Franssen and Kinzelbach, 2009) and the ease of combining the EnKF with virtually any forward model.

The EnKF is known to provide an optimal solution when the parameter vector follows a multiGaussian distribution and the state transfer function is linear (Arulampalam et al., 2002). The fact is that, in many practical applications of groundwater modeling, hydraulic conductivity cannot be modeled as multiGaussian distributed. The importance of accounting for nonmultiGaussinity and the impact of not accounting for it has been clearly demonstrated (e.g., Journel and Deutsch, 1993; Gómez-Hernández and Wen, 1998; Zinn and Harvey, 2003). Zhou et al. (2011) developed a new approach, the normal-score EnKF (NS-EnKF), to better handle non-Gaussianity distributions of hydraulic conductivity and states. Both, states and parameters, are normal-score transformed at each time step so that they follow marginal univariate Gaussian distributions. Then, the EnKF is applied on the normalscore transformed states and parameters. The performance of the NS-EnKF was shown to outperform the standard EnKF in a synthetic test example (Zhou et al., 2011).

The purpose of this paper is to evaluate the performance of the NS-EnKF in identifying hydraulic conductivity patterns in a bimodal aquifer by integrating dynamic hydraulic head data. The NS-EnKF has proven to work well in the presence of a relatively dense sample of hydraulic conductivities, i.e., direct measurements or hard data (Zhou et al., 2011). However, in practice we 
have to confront the situation in which the direct measurements of hydraulic conductivity are scarce or simply unavailable. Therefore we want to investigate the performance of the NS-EnKF to characterize a non-multiGaussian hydraulic conductivity distribution without the use of hard data. Hydraulic heads serve as the only information source, based on which to characterize the hydraulic conductivity model, and direct measurements of hydraulic conductivity are not considered in this experiment.

The structure of the remaining part is outlined as follows. The NS-EnKF algorithm is briefly introduced in Section 4.2. The method is tested in Section 4.3 on a synthetic bimodal aquifer composed of sand and shale, where the model is constrained to the observed hydraulic heads through the NS-EnKF and the standard EnKF. Results and discussions follow the synthetic example. The paper ends with some conclusions.

\subsection{The NS-EnKF}

The standard EnKF algorithm is described by Evensen (1994), Burgers et al. (1998) and Evensen (2007). The first application of the augmented EnKF in hydrogeology was applied by Chen and Zhang (2006). Details about the NS-EnKF algorithms can be found in Zhou et al. (2011). The main steps of the NS-EnKF algorithm are the same as for the standard EnKF, but the big difference is the introduction of additional pre- and post- processing steps carried out on the states and parameters contained in the augmented state vector. It starts with an ensemble of realizations that have been generated following a given random function. The NS-EnKF method consists of the following four main steps:

1. Forward simulation. For each realization of the ensemble, the state vector at time $t-1$ is updated to time $t$ using a state transfer equation, in our case, piezometric heads are updated from time $t-1$ to time $t$ through the solution of the groundwater flow equation, the hydraulic conductivities, which are members of the augmented state vector, remain unchanged through this step.

2. Normal score transformation. Establish, through the ensemble, the local cumulative distribution function (CDFs) at each grid cell for all the components of the augmented state vector (i.e., both states and parameters). These local CDFs are used to construct normal score transform functions, which are used to transform the augmented state vector into a new vector. All the components of the new vector follow a marginal Gaussian distribution with zero mean and unit variance. 
3. Update. The state vector is updated similarly as in the standard EnKF:

$$
\mathbf{x}_{t}^{u}=\mathbf{x}_{t}^{f}+\mathbf{G}_{t}\left(\mathbf{z}_{t}+\varepsilon-\mathbf{H x}_{t}^{f}\right)
$$

where $\mathbf{x}_{t}^{u}$ is the vector with the updated state variables at time $t$ (in normal space) and $\mathbf{x}_{t}^{f}$ is the vector computed from the forward simulation and then normal score transformed; $\mathbf{G}_{t}$ is the Kalman gain, derived on the basis of the minimization of the posterior error covariance; $\mathbf{z}_{t}$ is the normal-score transformed observation at time $t ; \varepsilon$ is an observation error characterized by a normal distribution with zero mean and a diagonal covariance (it is assumed that errors at different measurement locations are independent); $\mathbf{H}$ is the observation matrix.

4. Back transform. The updated state vector $\mathbf{x}_{t}^{u}$ is back transformed using the previously constructed local CDFs.

The above steps loop until all the observed piezometric heads (for a certain time period) are integrated.

\subsection{Synthetic example}

A synthetic bimodal aquifer composed of sand and shale occupies an area of $300 \mathrm{~m} \times 240 \mathrm{~m}$, where the hydraulic conductivities are characterized by a nonGaussian distribution and exhibit a range over several orders of magnitude. The study domain is discretized into 100 columns by 80 rows (the grid cells have dimensions of $3 \mathrm{~m} \times 3 \mathrm{~m}$ ). The aquifer is assumed confined with a thickness of $10 \mathrm{~m}$.

The reference facies field is generated by SNESIM (Strebelle, 2002), a multiple-point geostatistical simulation algorithm, using as training image the one in Figure 4.1. Each facies is then populated with log-conductivity values generated by GCOSIM3D (Gómez-Hernández and Journel, 1993) with parameters shown in Table 4.1. The reference $\ln K$ field is presented in Figure 4.2. The histogram of log-conductivity is bimodal, with modes coinciding with the means of the sand and shale distributions, $3.0 \ln (\mathrm{m} / \mathrm{d})$ and $-2.0 \ln (\mathrm{m} / \mathrm{d})$, respectively, and it has a global mean of $-0.47 \ln (\mathrm{m} / \mathrm{d})$ and a global standard deviation of $2.39 \ln (\mathrm{m} / \mathrm{d})$.

The groundwater flow equation is solved for the reference field using the transient flow simulator MODFLOW (Harbaugh et al., 2000) with impermeable boundary conditions in the north and south, constant head of $0 \mathrm{~m}$ in the west and prescribed flow rate of $270.5 \mathrm{~m}^{3} / \mathrm{d}$ along the east boundary distributed as can be seen in Figure 4.2. Different flow rates are set on the eastern boundaries depending on their water supply capacity, i.e., large flow rates correspond to zones with high conductivities. The initial head over the 


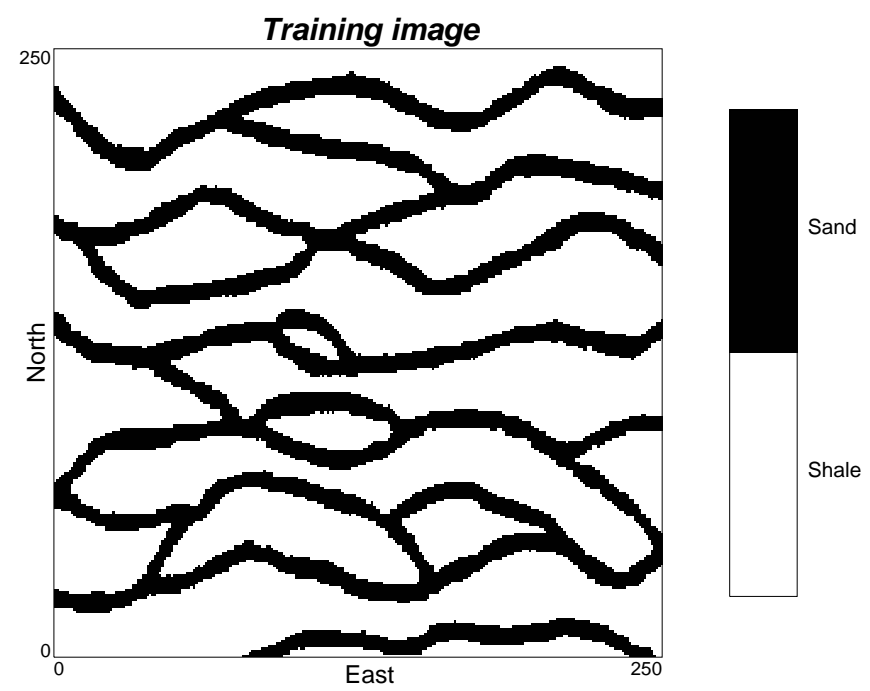

Figure 4.1: Training image used to generate the binary facies realizations (Strébelle, 2000).

Table 4.1: Parameters defining the multi-Gaussian random function used to generate the log-conductivity within each of the two facies.

\begin{tabular}{clllccc}
\hline Facies & $\begin{array}{l}\text { Variogram } \\
\text { type }\end{array}$ & $\begin{array}{l}\text { Mean } \\
(\ln (\mathrm{m} / \mathrm{d}))\end{array}$ & $\begin{array}{l}\text { Variance } \\
\left(\ln (\mathrm{m} / \mathrm{d})^{2}\right)\end{array}$ & $\lambda_{x}^{*}(\mathrm{~m})$ & $\lambda_{y}^{*}(\mathrm{~m})$ & Sill \\
\hline sand & exponential & 3.0 & 1.0 & 144 & 72 & 1.0 \\
shale & exponential & -2.0 & 1.0 & 72 & 72 & 0.35 \\
\hline
\end{tabular}

$*$ ranges in the $x$ and $y$ directions. 

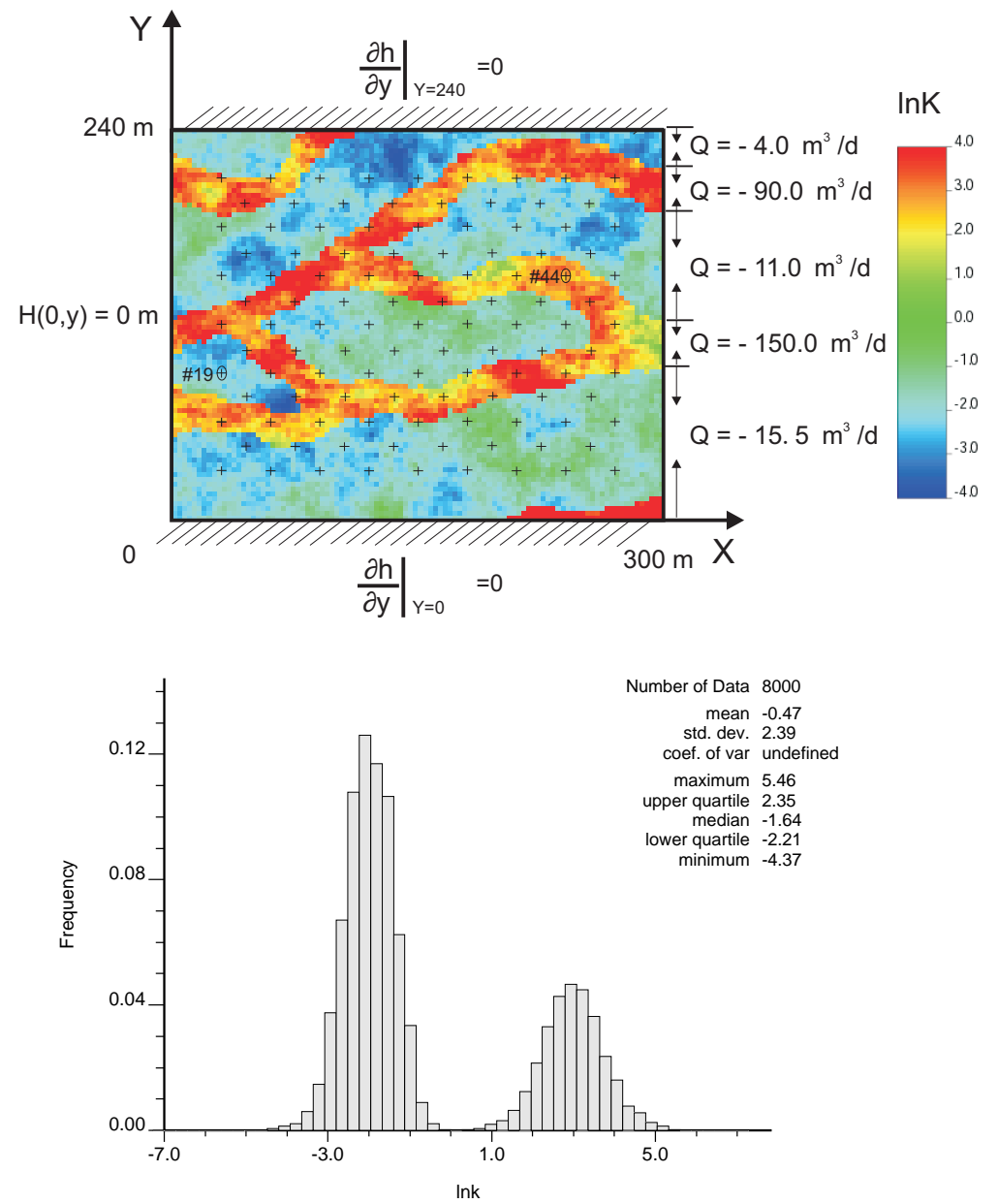

Figure 4.2: Reference log-conductivity field and the histogram. Flow boundary conditions and locations of piezometers are also indicated. 
domain is $0 \mathrm{~m}$. The total simulation period of 500 days is discretized into 100 steps with step sizes that increase following a geometric series with a ratio of 1.05. Specific storage is assumed constant and equal to be $0.003 \mathrm{~m}^{-1}$.

Using the same training image (Figure 4.1) and the same multiple-point geostatistical algorithm (SNESIM), 1000 facies realizations are generated. For each realization both facies are populated with $\ln K$ values generated with sequential Gaussian simulations using the parameters as specified in Table 4.1. The assimilation of piezometric head data is performed by the standard EnKF and the NS-EnKF. Observed piezometric heads from 111 piezometers in 60 time steps (67.7 days) serve as conditioning data. The locations of the 111 piezometers are shown in Figure 4.2.

\subsection{Results and discussions}

\subsubsection{Spatial variety characterization of $\ln K$}

Figure 4.3 shows the evolution of the ensemble mean and variance of logconductivity as the piezometric head data are assimilated by the NS-EnKF from the initial time step $(t=0)$ to the end of the data assimilation $(t=60)$. The ensemble mean of the initial realizations is smooth and shows no feature even though each initial realization honors the same multiple-point geostatistical model implicit in the training image. Structures of the spatial pattern of $\ln K$ start to appear during the conditioning to piezometric head data. For instance, at the $5^{\text {th }}$ assimilation step, the channel pattern near the eastern boundary is identified and at the end of the $60^{\text {th }}$ step, the ensemble mean of $\ln K$ resembles closely the reference field, having identified quite precisely the channel locations in the reference. The variance is also reduced over the domain as more piezometric heads are integrated. At the end of time step 60 we can even identify the boundaries of the channels by the largest variance strings. We can argue that assimilation of hydraulic heads with the NS-EnKF plays a critical role in recognizing patterns of $\ln K$ and allows a good characterization of the bimodal aquifer.

Figure 4.4 shows the results obtained using the standard EnKF. The ensemble mean is similar to the one obtained by the NS-EnKF, i.e., the $\ln K$ patterns are recognized as more piezometric heads are assimilated. The main difference resides in the variance field. The variance is initially reduced around the piezometers, which is clearly illustrated by observing the variance field up to the $15^{\text {th }}$ time step while in the case of NS-EnKF, the influence area of the piezometer is extended and depends on the channels to a large extent. Then, at the end of data assimilation $(t=60)$, the $\ln K$ variance in Figure 4.4 is widely reduced over the entire domain. On one hand, the variance reduction is what we expect as additional information is incorporated in the model; on 


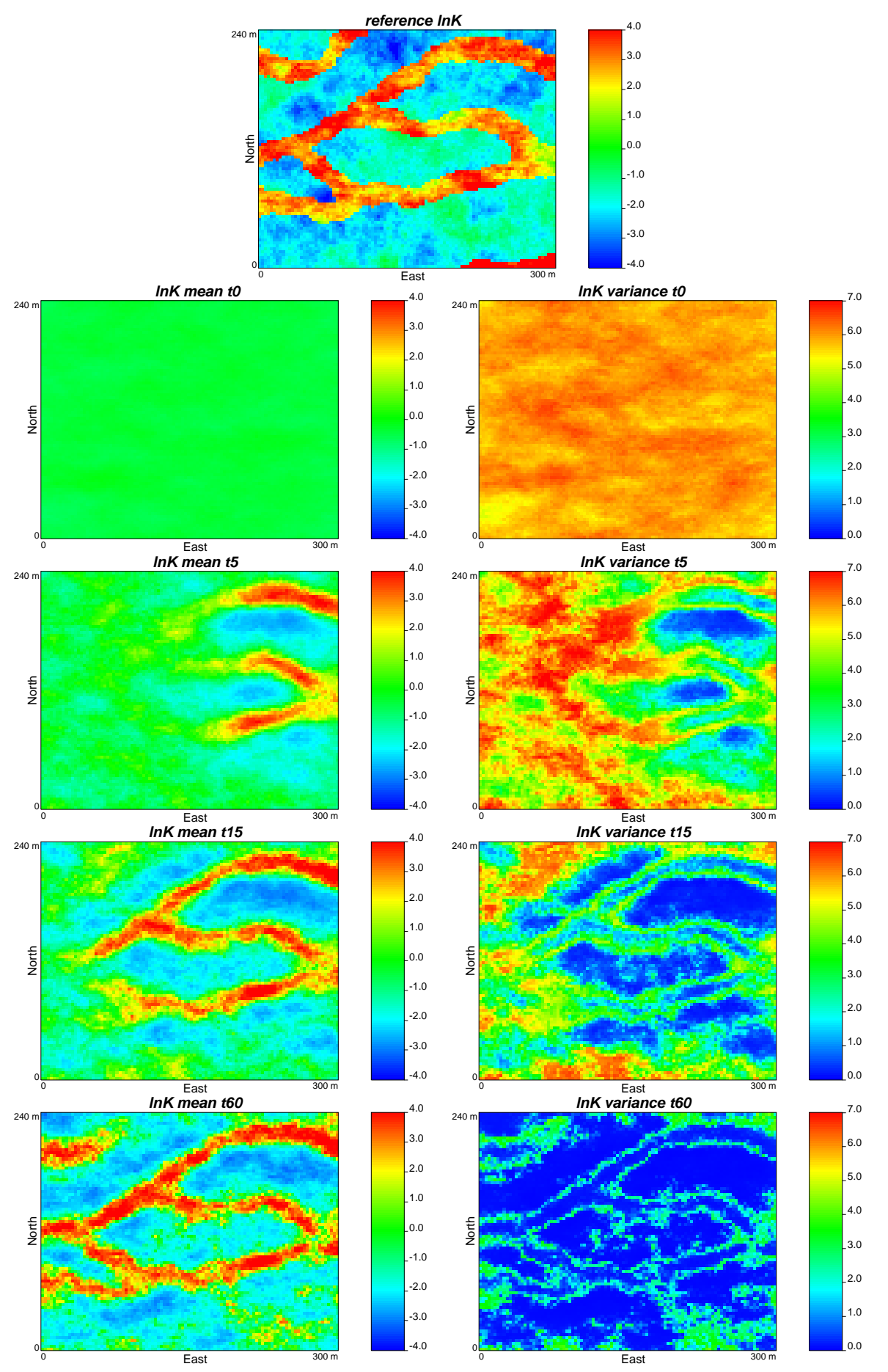

Figure 4.3: Evolution of the $\ln K$ ensemble mean and variance as data are assimilated with the NS-EnKF for time steps 5, 15 and 60 . Reference $\ln K$ is shown for comparison. 
the other hand, the over-reduction of the ensemble variance cannot represent the real uncertainty, that is, the uncertainty is underestimated. This can be reinforced by Figure 4.5 which shows the evolution of the average absolute bias $\left(A A B(x)_{t}\right)$ and the average ensemble spread $\left(A E S P(x)_{t}\right)$.

The $A A B(x)_{t}$ and the $A E S P(x)_{t}$ evaluate accuracy and uncertainty of the estimation, respectively. They are defined as

$$
\begin{aligned}
A A B(x)_{t} & =\frac{1}{N_{b}} \sum_{i=1}^{N_{b}} \frac{1}{N_{e}} \sum_{r=1}^{N_{e}}\left|x_{t, i, r}-x_{r e f, i}\right| \\
\operatorname{AESP}(x)_{t} & =\left(\frac{1}{N_{b}} \sum_{i=1}^{N_{b}} \sigma_{x_{t, i}}^{2}\right)^{1 / 2}
\end{aligned}
$$

where $x_{t, i, r}$ is the estimated log-conductivity at time step $t$, node $i$ and realization $r, x_{r e f, i}$ is the reference log-conductivity at node $i, N_{b}$ is the number of nodes, $N_{e}$ is the number of realizations and $\sigma_{x_{t, i}}^{2}$ is the variance over the ensemble at time step $t$ and location $i$. We can see that as assimilation time advances, the $A A B(x)_{t}$ and the $A E S P(x)_{t}$ decrease and approach a stable value. Clearly, the $A A B(x)_{60}$ corresponding to the NS-EnKF is smaller than that for the standard EnKF, indicating a more accurate estimation. On the contrary, the $\operatorname{AESP}(x)_{60}$ corresponding to the NS-EnKF is bigger than that for the standard EnKF, indicating an estimate with higher uncertainty. The discrepancy discrepancy between $A A B(x)_{60}$ and $A E S P(x)_{60}$ is much larger for the standard EnKF than for NS-EnKF, which indicates that the former underestimation the uncertainty in relation with the latter.

Figure 4.6 displays $\ln K$ histograms of the reference field, the prior ensemble and the updated ensemble by the EnKF and the NS-EnKF after piezometric heads are assimilated. The global mean and standard deviation are preserved during data assimilation for both the standard EnKF and the NSEnKF. The bimodal histogram for the prior ensemble (related with the sand and shale facies) is correctly preserved by the NS-EnKF, but it is not during data assimilation with the standard EnKF. In the latter case, the updated histogram tends to be Gaussian and the bimodality is almost gone. Besides, the histogram obtained with the standard EnKF shows extremely high and low values, outside of the range of the reference histogram.

Connectivity plays a key role in solute transport simulation and thus a series of measures has been proposed to assess it. Here we adopt the connectivity function which measures the probability that two points within the same facies are connected by a continuous path (Stauffer and Aharony, 1994). We focus on the connectivity of sand along the $x$ direction which is the main orientation of the channels. The log-conductivity values are first converted to 


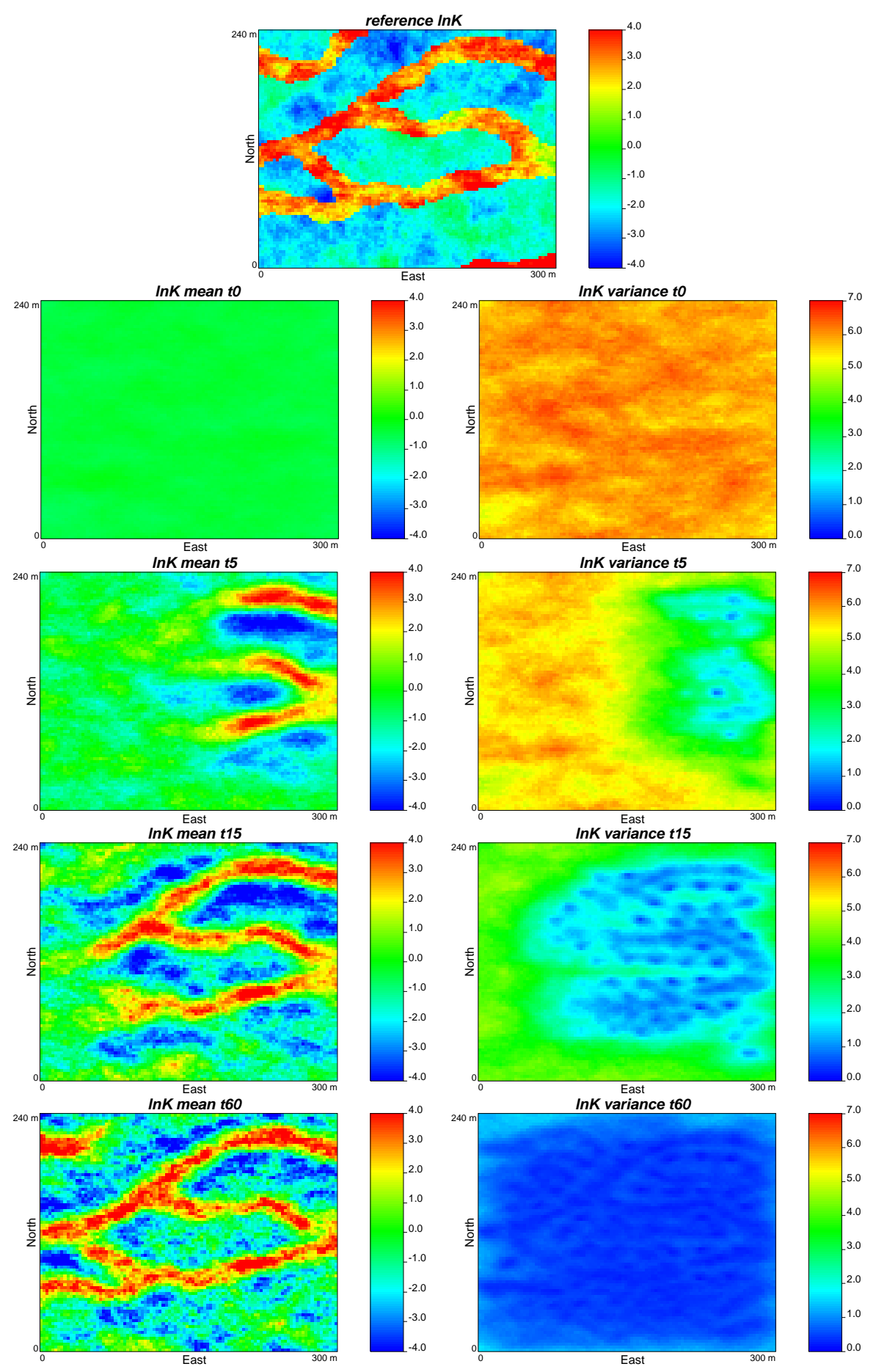

Figure 4.4: Evolution of the $\ln K$ ensemble mean and variance as data are assimilated with standard EnKF for time steps 5, 15 and 60. Reference $\ln K$ is shown for comparison. 


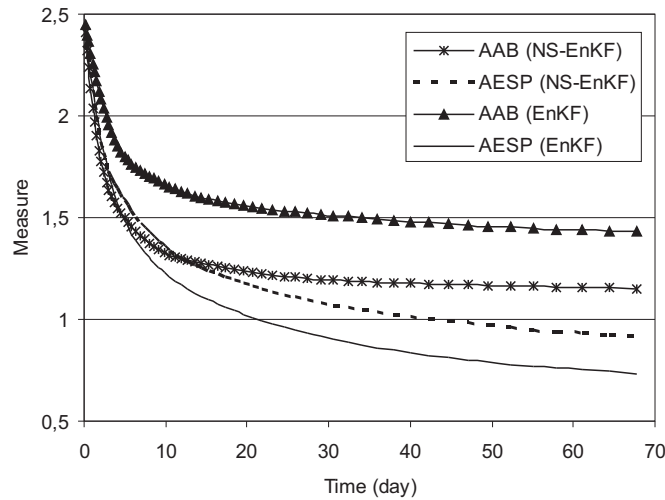

Figure 4.5: Evolution of Average Absolute Bias $\mathrm{AAB}(\ln K)$ and Average Ensemble Spread $\operatorname{AESP}(\ln K)$ for sequential data assimilation with standard EnKF and the NS-EnKF.
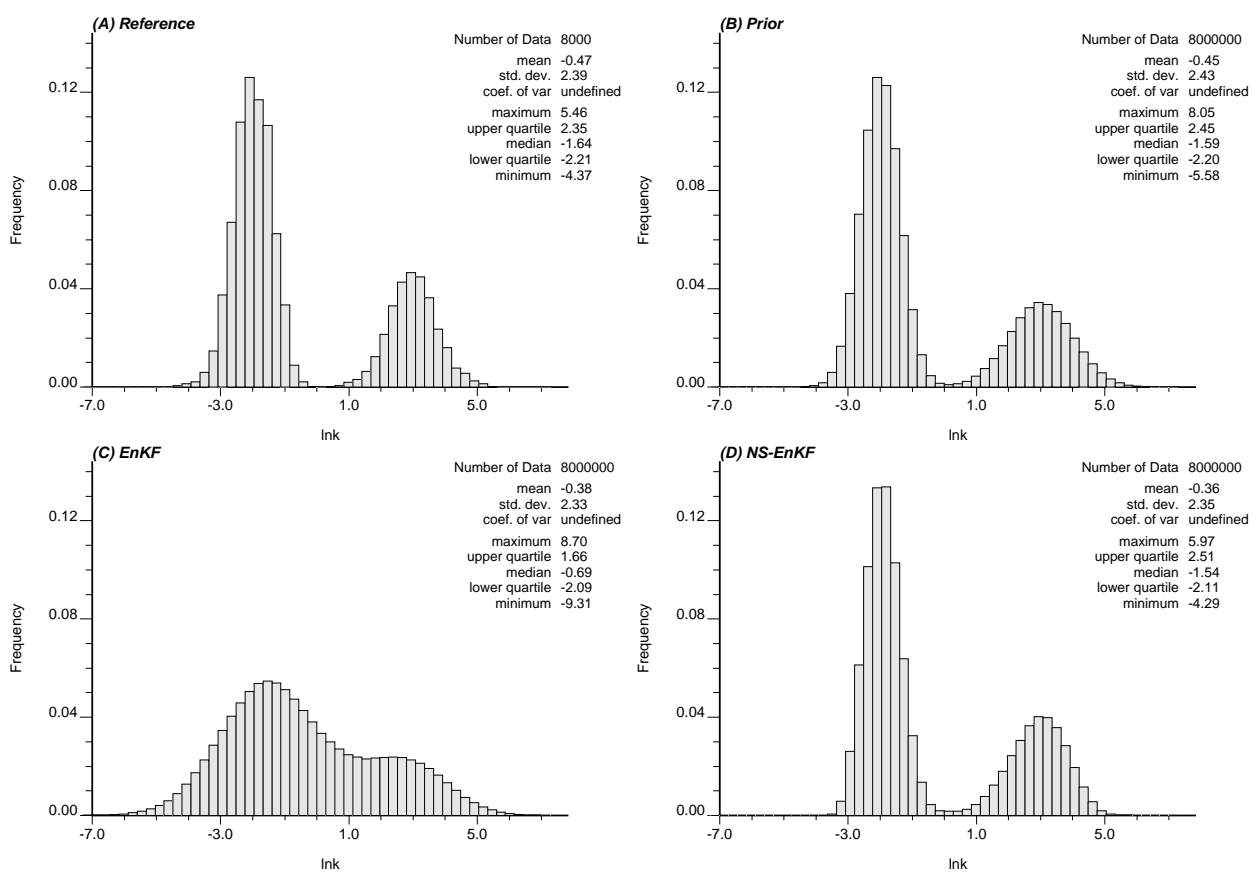

Figure 4.6: $\ln K$ histograms for the (A) reference, (B) prior, (C) posterior with the standard EnKF, and (D) posterior with the NS-EnKF. 
indicator variables according to

$$
I(x)= \begin{cases}1, & \text { if } \ln K \geq 0 \\ 0, & \text { otherwise }\end{cases}
$$

which, in this particular case, is equivalent to partition them into sand and shale. Then, the program CONNEC3D (Pardo-Igúzquiza and Dowd, 2003) is used to compute the connectivity functions for different distance lags for log-conductivities larger than $0 \ln (\mathrm{m} / \mathrm{d})$. Figure 4.7 shows the connectivity function changes with distance before (Figure 4.7A) and after data assimilation with the standard EnKF (Figure 4.7B) and the NS-EnKF (Figure 4.7C). These figures show the functions for the reference field, for each updated stochastic realization and they also show the mean of the connectivity functions over the stochastic realizations. We can see that the reference connectivity function is underestimated by both the standard EnKF and the NS-EnKF, but the latter performs a little better, the span of the ensemble of realizations includes almost completely the connectivity function for the reference field.

\subsubsection{Prediction capacity of updated $\ln K$}

The previous assessments focused on the estimation of log-conductivity itself, for this purpose, the NS-EnKF is found to perform properly in detecting the log-conductivity pattern, preserving the bimodal histogram and estimating the connectivity. Now, the updated log-conductivity realizations will be tested for their ability to perform predictions.

Figure 4.8 shows the hydraulic head evolution with time at two of the piezometers, one located in shale (Piezometer \#19) and the other in sand (Piezometer \#44). Hydraulic head predictions for the prior and updated realizations are displayed. Data assimilation by the standard EnKF and the NS-EnKF result in a significant reduction of prediction uncertainty for both methods at both piezometers. The accuracy of hydraulic head prediction is very similar for the NS-EnKF and for the standard EnKF although the updated $\ln K$ fields by NS-EnKF are superior in terms of detection of the spatial patterns of hydraulic conductivities. This fact can be attributed to the smoothing effect of the groundwater flow equation (Delhomme, 1979), i.e., similar piezometric head distributions can be obtained for different $\ln K$ fields, and thus the non-uniqueness of solutions to the inverse problem (Carrera and Neuman, 1986).

To further evaluate the updated $\ln K$ fields we performed a transport prediction experiment for the case of steady-state flow. Figure 4.9 shows the setup of this synthetic experiment. No flow boundary conditions are defined for the northern and southern boundary and prescribed heads along the western 

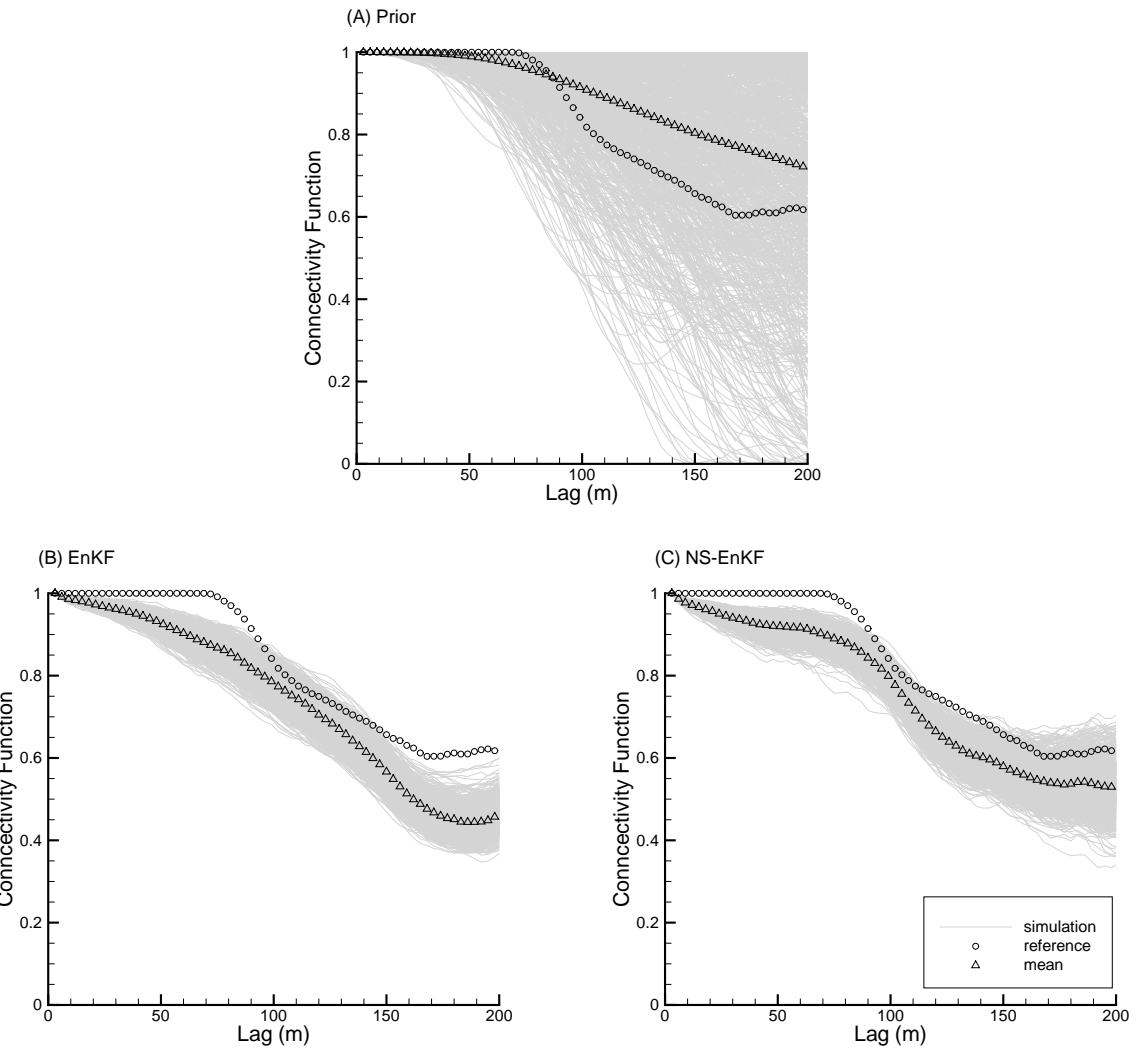

Figure 4.7: Connectivity as a function of the separation distance, measuring the probability that two points in $x$ direction are connected by a continuous path of $\ln K$ larger than $0 \ln (\mathrm{m} / \mathrm{d})$. Shown are the results for $(\mathrm{A})$ the prior unconditional realizations, (B) the realizations conditioned with standard EnKF, and $(\mathrm{C})$ the realizations conditioned with the NS-EnKF. 

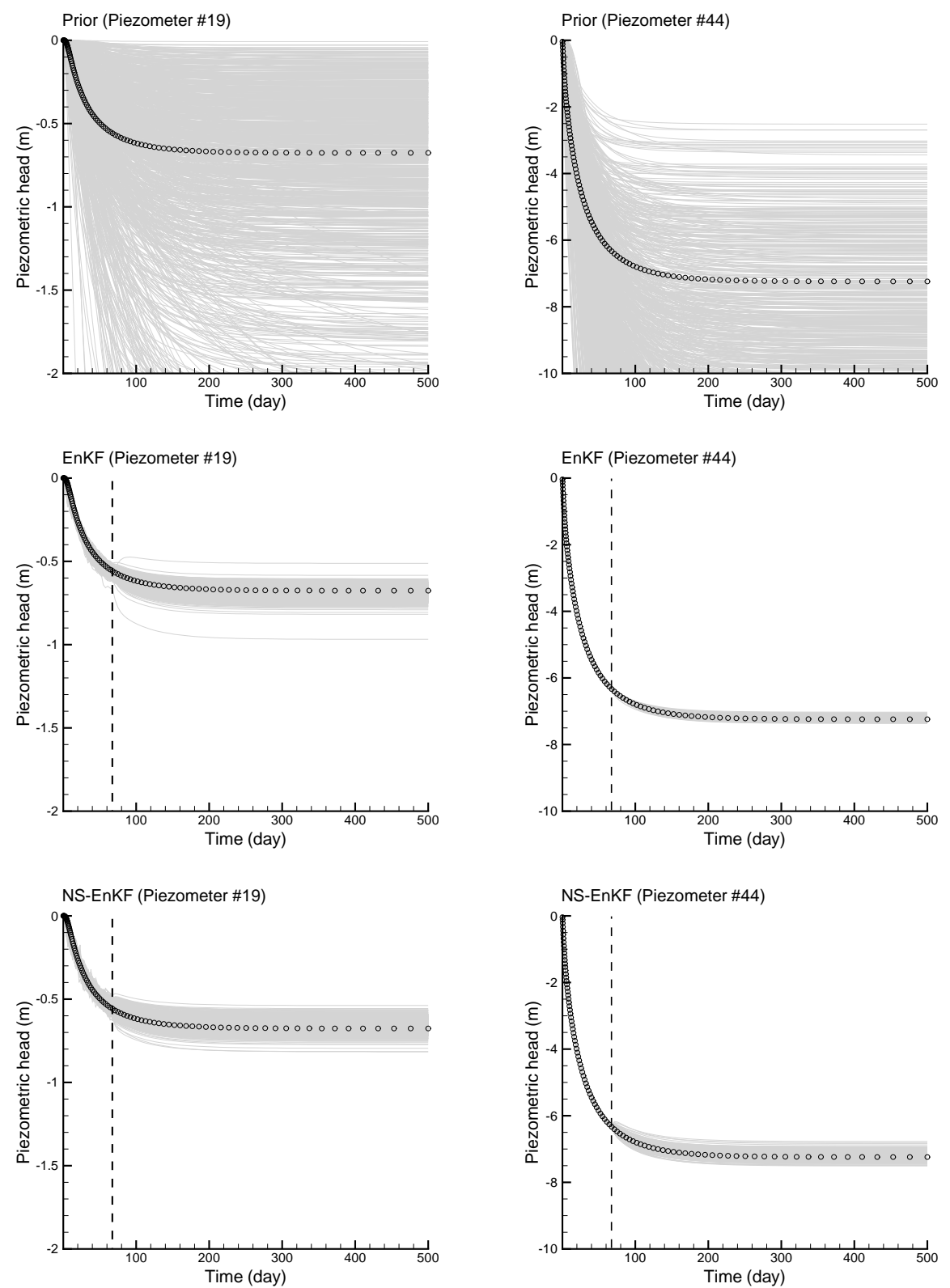

Figure 4.8: Piezometric head evolution for two of the 111 piezometers, locations of which are indicated in Figure 4.2. Results are shown for the prior ensemble (no data assimilation), for assimilation with the standard EnKF and the NS-EnKF (conditioning to piezometric heads until 67.7 days, indicated by the vertical dashed line). Circles refer to piezometric head in the reference field. 
(equal to $0 \mathrm{~m}$ ) and eastern boundary (equal to $-10 \mathrm{~m}$ ). Conservative particles are released along a vertical line at $x=19.5 \mathrm{~m}$ and two control planes are located at $x=110 \mathrm{~m}$ and $x=290 \mathrm{~m}$, at which the arrival times are recorded. The random walk particle tracking program RW3D (Fernàndez-Garcia et al., 2005; Li et al., 2011) is used to solve the conservative transport equation. The integrated breakthrough curves (BTCs) at the two control planes are computed and compared with the prior BTCs and the reference BTCs (Figure 4.10). For both the standard EnKF and the NS-EnKF, the uncertainty of BTC prediction is significantly reduced, indicating the importance to integrate piezometric head data. With standard EnKF, the bias and uncertainty are reduced compared with the prior at both control planes. However, the ensemble median deviates from the reference and the reference is not enclosed in the $90 \%$ confidence interval. With the NS-EnKF, not only the bias and prediction uncertainty are significantly reduced at both control planes but also the reference is well represented by the ensemble median, and it is contained within the $90 \%$ confidence interval, especially for control plane B.

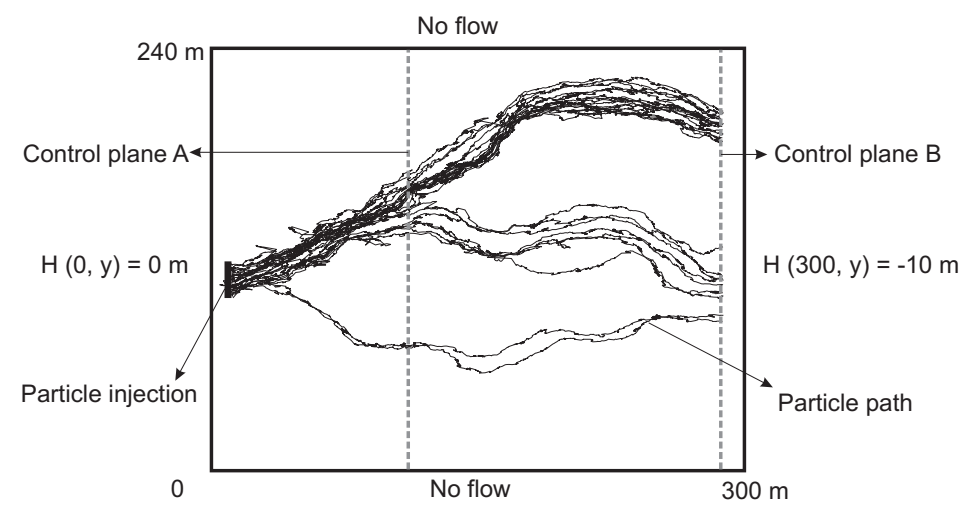

Figure 4.9: Configuration of the transport prediction experiment. Shown are boundary conditions, locations for particle injection, location of the two control planes and an illustration of the particle paths.

\subsection{Conclusions}

The EnKF has been widely used as a data assimilation algorithm to estimate model parameters and update model states. The EnKF has gained popularity in various disciplines due to its attractive advantages in comparison with other inverse methods in such respect as uncertainty characterization, computational efficiency and flexibility. However, EnKF does not perform optimally for non-multiGaussian parameter distributions and nonlinear models. The normal-score EnKF (NS-EnKF) was proposed (Zhou et al., 2011) in order to 

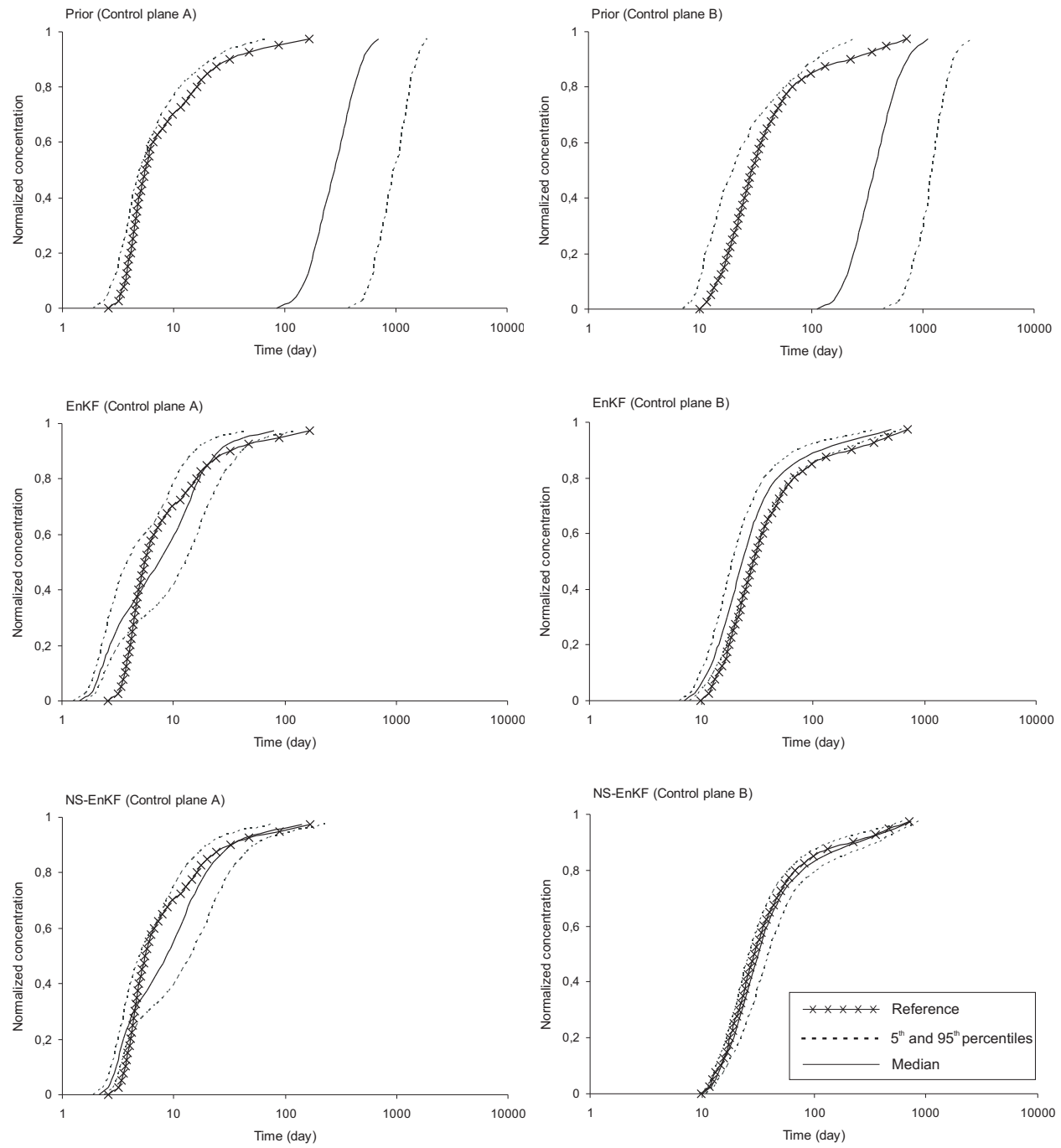

Figure 4.10: Breakthrough curves at two control planes for the prior ensemble and the ensemble updated with the standard EnKF and the NS-EnKF. The $5^{\text {th }}$ percentile, median, $95^{\text {th }}$ percentile and reference of the breakthrough curves are shown. 
reduce problems related with non-Gaussianity of states and parameters. A normal-score transformation is applied so that the marginal distributions of states and parameters are Gaussian. The objective of this paper is to investigate the behavior of the NS-EnKF in identifying $\ln K$ patterns for a synthetic non-Gaussian aquifer with a bimodal $\ln K$ distribution by assimilating hydraulic heads in the absence of $\ln K$ measurements. The standard EnKF is also used for the same synthetic set-up in order to compare the results. The NS-EnKF gives better results than the standard EnKF because: (1) the bimodal histogram is well preserved by the NS-EnKF while it is not by the standard EnKF, (2) parameter uncertainty is underestimated by the standard EnKF with respect to the NS-EnKF, (3) channel connectivity along the $x$ direction is underestimated by both methods but the connectivity functions computed on the ensemble of realizations obtained by NS-EnKF are closer to the connectivity function in the synthetic reference field, and (4) the fate of conservative solute is predicted correctly by the updated $\ln K$ fields in NSEnKF while the results by standard EnKF exhibit a certain deviation from the reference. In conclusion, piezometric head data carry important information which, in conjunction with a knowledge of the prior histogram of the hydraulic conductivity, permits the characterization of a non-Gaussian $\ln K$ distribution even if no hydraulic conductivity data are available. 


\section{Bibliography}

Arulampalam, M. S., Maskell, S., Gordon, N., Clapp, T., 2002. A tutorial on particle filters for online nonlinear/non-Gaussian Bayesian tracking. IEEE Transactions on signal processing 50 (2), 174-188.

Bertino, L., Evensen, G., Wackernagel, H., 2003. Sequential data assimilation techniques in oceanography. International Statistical Review 71 (2), 223241.

Burgers, G., Jan van Leeuwen, P., Evensen, G., 1998. Analysis scheme in the ensemble Kalman filter. Monthly Weather Review 126 (6), 1719-1724.

Carrera, J., Neuman, S. P., 1986. Estimation of aquifer parameters under transient and steady state conditions: 2 . uniqueness, stability, and solution algorithms. Water Resources Research 22 (2), 211-227.

Chen, Y., Zhang, D., 2006. Data assimilation for transient flow in geologic formations via ensemble Kalman filter. Advances in Water Resources 29 (8), $1107-1122$.

Delhomme, J. P., 1979. Spatial variability and uncertainty in groundwater flow parameters: A geostatistical approach. Water Resources Research 15 (2), 269-280.

Evensen, G., 1994. Sequential data assimilation with a nonlinear quasigeostrophic model using Monte Carlo methods to forecast error statistics. Journal of Geophysical Research 99 (C5), 10143-10162.

Evensen, G., 2007. Data assimilation: The ensemble Kalman filter. Springer Verlag, 279pp.

Fernàndez-Garcia, D., Illangasekare, T., Rajaram, H., 2005. Differences in the scale dependence of dispersivity and retardation factors estimated from forced-gradient and uniform flow tracer tests in three-dimensional physically and chemically heterogeneous porous media. Water Resources Research 41 (3), W03012. 
Gómez-Hernández, J. J., Journel, A. G., 1993. Joint sequential simulation of Multi-Gaussian fields. In: Soares, A. (Ed.), Geostatistics Tróia '92. Vol. 1. Kluwer Academic Publishers, Dordrecht, pp. 85-94.

Gómez-Hernández, J. J., Wen, X. H., 1998. To be or not to be multi-Gaussian? A reflection on stochastic hydrogeology. Advances in Water Resources 21 (1), $47-61$.

Harbaugh, A. W., Banta, E. R., Hill, M. C., McDonald, M. G., 2000. MODFLOW-2000, the U.S. geological survey modular ground-water model - user guide to modularization concepts and the ground-water flow process. Tech. Rep. Open-File Report 00-92, U.S. Department of the Interior, U.S. Geological Survey, Reston, Virginia, 121pp.

Hendricks Franssen, H. J., Kinzelbach, W., 2008. Real-time groundwater flow modeling with the Ensemble Kalman Filter: Joint estimation for states and parameters and the filter inbreeding problem. Water Resources Research 44, W09408.

Hendricks Franssen, H. J., Kinzelbach, W., 2009. Ensemble Kalman filtering versus sequential self-calibration for inverse modelling of dynamic groundwater flow systems. Journal of Hydrology 365 (3-4), 261-274.

Houtekamer, P. L., Mitchell, H. L., 2001. A sequential ensemble Kalman filter for atmospheric data assimilation. Monthly Weather Review 129 (1), 123137.

Journel, A. G., Deutsch, C. V., 1993. Entropy and spatial disorder. Mathematical Geology 25 (3), 329-355.

Li, L., Zhou, H., Gómez-Hernández, J. J., 2011. A comparative study of threedimensional hydraulic conductivity upscaling at the macrodispersion experiment (MADE) site, on columbus air force base in mississippi (USA). Journal of Hydrology, doi:10.1016/j.jhydrol.2011.05.001.

Moradkhani, H., Sorooshian, S., Gupta, H. V., Houser, P. R., 2005. Dual state-parameter estimation of hydrological models using ensemble Kalman filter. Advances in Water Resources 28 (2), 135-147.

Nævdal, G., Johnsen, L., Aanonsen, S., Vefring, E., 2005. Reservoir monitoring and continuous model updating using ensemble kalman filter. SPE journal $10(1), 66-74$.

Pardo-Igúzquiza, E., Dowd, P. A., 2003. CONNEC3D: a computer program for connectivity analysis of $3 \mathrm{D}$ random set models. Computers \& Geosciences 29, 775-785. 
Stauffer, D., Aharony, A., 1994. Introduction to percolation theory. Taylor and Francis, London. 181pp.

Strébelle, S., 2000. Sequential simulation drawing structures from training images. Ph.D. thesis, Stanford University. 187pp.

Strebelle, S., 2002. Conditional simulation of complex geological structures using multiple-point statistics. Mathematical Geology 34 (1), 1-21.

Wen, X., Chen, W., 2005. Real-time reservoir model updating using ensemble Kalman filter. SPE 92991.

Zhou, H., Gómez-Hernández, J. J., Hendricks Franssen, H.-J., Li, L., 2011. An approach to handling Non-Gaussianity of parameters and state variables in ensemble Kalman filter. Advances in Water Resources, doi:10.1016/j.advwatres.2011.04.014.

Zinn, B., Harvey, C., 2003. When good statistical models of aquifer heterogeneity go bad: A comparison of flow, dispersion, and mass transfer in connected and multivariate Gaussian hydraulic conductivity fields. Water Resources Research 39 (3), 1051. 
Water Resources Research, under review.

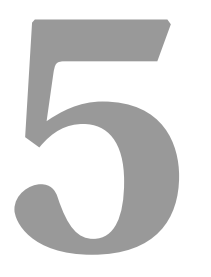

\title{
A Pattern Search Based Inverse Method
}

\begin{abstract}
Uncertainty of model predictions is caused to a large extent by the uncertainty on model parameters while the identification of model parameters is demanding due to the inherent heterogeneity of the aquifer. A variety of inverse methods has been proposed for model identification. In this paper we present a novel inverse method to constrain the model parameters (hydraulic conductivities) to the observed hydraulic head data. In the method proposed we build a conditioning pattern consisting of simulated model parameters and observed flow data. The unknown parameter values are simulated by pattern searching through an ensemble of realizations rather than optimizing an objective function. The model parameters do not necessarily follow a multiGaussian distribution and the nonlinear relationship between the parameter and response is captured by the multipoint pattern matching. The algorithm is evaluated in two synthetic bimodal aquifers. The proposed method is able to reproduce the main structure of the reference fields and the performance of the updated model in predicting flow and transport is improved compared with the prior model.
\end{abstract}




\section{$5.1 \quad$ Introduction}

The inverse problem in hydrogeology aims to gain understanding about the characteristics of the subsurface, i.e., identification of model structure and corresponding parameters, by integrating observed model responses such as hydraulic head and mass concentration data. Several inverse methods have been proposed to solve the inverse problem in the last several decades. The first application of inverse methods in hydrogeology treat the aquifer properties as piecewise homogeneous (e.g., Carrera and Neuman, 1986). However, soon it is realized that the aquifer should be characterized by heterogeneous distributions of the parameters (see De Marsily et al., 2005, for a historic perspective on the treatment of heterogeneity in aquifer modeling). There are already several inverse methods capable of dealing with this heterogeneity, e.g., the pilot point method (RamaRao et al., 1995), the self-calibration method (Gómez-Hernández et al., 1997; Wen et al., 1999; Hendricks Franssen et al., 2003), the ensemble Kalman filter (Evensen, 2003; Chen and Zhang, 2006; Hendricks Franssen and Kinzelbach, 2008; Zhou et al., 2011) or the Markov chain Monte Carlo method (Oliver et al., 1997; Fu and Gómez-Hernández, 2009).

In the above referred inverse methods, the groundwater model structure is described by a variogram model, which basically measures the correlation between two spatial locations. This two-point variogram-based model is not able to characterize curvilinear features, e.g., cross-bedded structures in fluvial deposits or erosion fractures in karstic formations, while these curvilinear structures play a key role in flow and especially solute migration modeling (e.g., Kerrou et al., 2008; Li et al., 2011b). A solution to address this issue is to use multiple-point geostatistics. A "training image", which contains the types of features to be reproduced by the aquifer model, is introduced as a geological conceptual model (Guardiano and Srivastava, 1993). This training image is used to derive experimental local conditional distributions that serve to propagate the curvilinear patterns onto the simulated aquifer. A series of programs based on multiple-point geostatistics is available, e.g., SNESIM (Strebelle, 2002), FILTERSIM (Zhang et al., 2006), SIMPAT (Arpat and Caers, 2007) and DS (Mariethoz et al., 2010b), and a detailed review on multiple-point geostatistics is provided by $\mathrm{Hu}$ and Chugunova (2008). The advantages of using multiple-point geostatistics for the characterization of hydraulic conductivity and for flow and transport prediction have been confirmed after comparison with variogram-based simulation methods, both in synthetic examples and in real aquifers (e.g., Feyen and Caers, 2006; Huysmans and Dassargues, 2009; Journel and Zhang, 2006). An alternative to derive multiple-point statistics is using high-order spatial cumulants which are combinations of moments of 
spatial parameters to characterize non-Gaussian nonlinear random variables (Dimitrakopoulos et al., 2010; Mustapha and Dimitrakopoulos, 2010).

Most of the inverse methods construct an objective function to measure the deviation between the simulated and observed data. Then, through an optimization algorithm, the initial aquifer models are modified until the observed data are well reproduced by the model predictions. However, during the optimization process, the aquifer spatial structure may be modified with respect to the structure of the initial guesses and become geologically unrealistic (Kitanidis, 2007). To prevent this departure, techniques such as including a regularization term or using a plausibility criterion are combined with the objective function to constrain the deviation of the updated model from the prior model (Emsellem and De Marsily, 1971; Neuman, 1973). But these methods have been challenged on their theoretical foundations (RamaRao et al., 1995; Rubin et al., 2010). Some recent inverse methods use other avenues in an attempt to preserve the prior structure when perturbing the parameter values in the prior fields.

Considering the limits of the conventional inverse methods and the advantages of multiple-point geostatistics, a reasonable solution is using the multiple-point geostatistics to characterize the nonlinear structure and try to preserve the structure when the model is updated using inverse methods. In this way, the curvilinear features are characterized properly and the model remains physically realistic during the inverse process. A few examples of such inverse methods include the gradual deformation method (GDM) (Hu, 2000; Caers, 2003), the probability perturbation method (PPM) (Caers, 2002; Caers and Hoffman, 2006) and the probability conditioning method (PCM) (Jafarpour and Khodabakhshi, 2011). In all three methods, the prior model structure can be characterized by multiple-point statistics and the property realizations are updated in such a way that the prior model statistics are kept. The difference between the three methods resides in the way the observations are integrated and the way the realizations are updated. The main idea of the GDM is that the realizations are perturbed by modifying the random number used to draw from the conditional distribution function. The random number is chosen through optimizing the deformation parameter so that the mismatch between the simulated and observed dynamic data is reduced. The PPM is based in modifying the conditional probability function. For the case of PCM, the realizations are updated with a multiple-point simulation method under a soft constraint given by a probability map inferred from observed flow data. The probability map is built with the help of the ensemble Kalman filter.

Alternatively to the inverse methods formulated in the framework of minimizing an objective function, the Markov chain Monte Carlo method provides another way, namely sampling from a posterior distribution that is already conditioned to observations. Two such examples that are capable of dealing 
with curvilinear structures are the blocking moving window algorithm (Alcolea and Renard, 2010) and the iterative spatial resampling (Mariethoz et al., 2010a). Another avenue is treating the inverse problem as a search problem, e.g., a distance-based inverse method (Suzuki and Caers, 2008). A large number of multiple-point simulations are constructed, from which a search scheme is used to select those consistent with the observed dynamic data. The spatial structure of the parameters is not disturbed since no modification is performed, simply a selection is carried out. The updated model should be geologically realistic as long as the prior model is generated properly.

In this paper, we present a novel approach to constrain the hydraulic conductivities to dynamic flow data. The most distinct novelty of the proposed method is that we formulate the inverse problem in the basis of pattern search instead of minimizing an objective function or sampling the posterior distribution. We assume that the hydraulic conductivity to be simulated is related to the geologic structure and flow dynamics in its neighborhood. The value at the simulated node is determined by searching for matches to the conditional pattern composed of simulated hydraulic conductivities and observed flow data. The proposed pixel-based method is not only convenient to condition to local data but it is also able to describe the geologic structure. Furthermore, the pattern is searched through the prior ensemble of realizations all of which are consistent with the geologic structure so that the pattern-search method ensures that the updated fields are physically realistic and the prior statistics are preserved.

The rest of the paper is outlined as follows. In section 5.2, the proposed method is presented in detail. In section 5.3, a synthetic example is described to assess the performance of the method. In section 5.4, the results of the synthetic experiment are presented and analyzed. In section 5.5, the method is further evaluated with another example to test the effect of number of conditioning data and boundary conditions. In section 5.6, some conclusions about the proposed method are shown.

\subsection{Methodology}

The method is based on the direct sampling algorithm proposed by Mariethoz et al. (2010b). It has been extended to include state observation data, which forces the enlargement of the concept of training image to a training ensemble of realizations.

\subsubsection{Flow chart of the algorithm}

A flow chart of the proposed method is displayed in Figure 5.1, which consists of the following steps: 


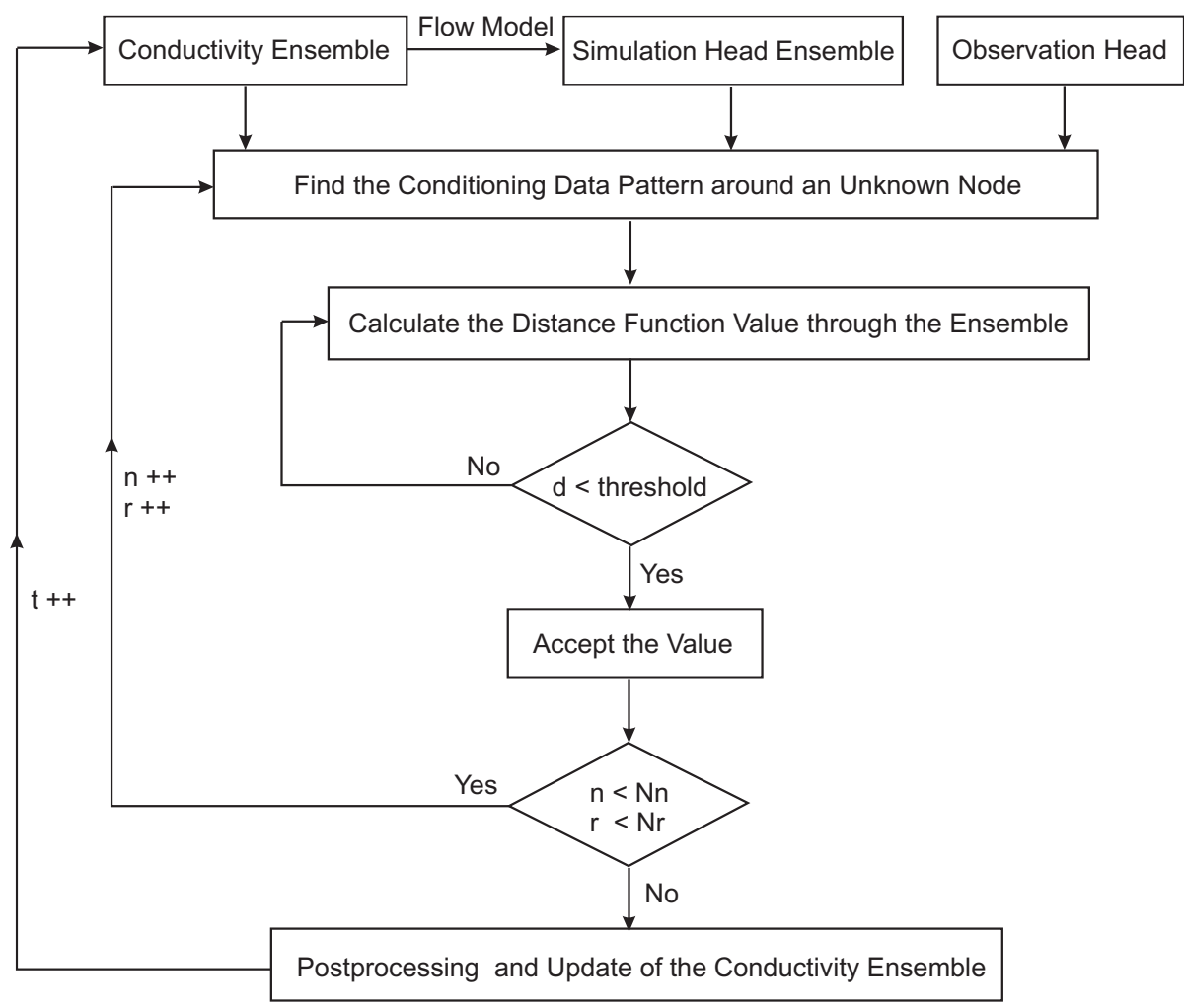

Figure 5.1: Flow chart of the proposed pattern-searching based multiple-point ensemble inverse method. 
- Generate the prior ensemble of realizations. For the purpose of illustration we will consider that hydraulic conductivity is the parameter of interest. Let the ensemble be composed of $N_{r}$ realizations and each hydraulic conductivity field be discretized into $N_{n}$ cells. Multiple-point sequential simulation methods are applied to generate the conductivity field ensemble, e.g., using the SNESIM or the DS codes mentioned in the previous section. A training image is needed for the generation. The hydraulic conductivity hard data are honored if available.

- Forecast the dependent state variables. For each realization, the hydraulic head data are obtained by solving the transient flow equation on the hydraulic conductivity field subject to initial and boundary conditions. We assume that the initial and boundary conditions are known perfectly so that we can focus on the uncertainty caused by hydraulic conductivities. Notice that only model parameters are updated. To avoid inconsistencies between the currently simulated hydraulic heads and the updated hydraulic conductivities, the flow simulation is restarted from the beginning whenever the hydraulic conductivity field is updated. At this stage we have an ensemble of hydraulic conductivity realizations that mimic the patterns of the training image, and the corresponding ensemble of piezometric head fields. These two ensembles will become now the training image in which to look for patterns that will permit the generation of conductivity fields which also respect the piezometric head measurements.

- For each realization, define a random path visiting each node except those with hydraulic conductivity measurements. For each node with an unknown value $\left(K_{i}\right)$ in the sequential path,

1. Determine the conditional data pattern of $K_{i}$. In this work, the data pattern is composed of hydraulic conductivities and piezometric heads. The conditional hydraulic conductivities include measured hard data, if any, and previously simulated values. The known hydraulic conductivities and observed heads around $K_{i}$ are ranked according to distance. A maximum number $M$ of conditional hydraulic conductivities and a maximum number $N$ of conditional piezometric heads are set. Only the closest $M$ hydraulic conductivities and the closest $N$ observed heads are stored as conditional data constituting the conditioning pattern. For instance, in Figure 5.2, the conditioning data pattern for $K_{i}$ consists of three hydraulic conductivities and two observed heads. The size of the conditional data pattern is not determined by the search area but instead by the number of conditioning data. The varying-size search 
neighborhood scheme was proposed by Mariethoz et al. (2010b). Advantages of this pattern configuration are two-fold: (a) the size of the conductivity data event in the pattern is influenced by the density of the known conductivities, i.e., when the known conductivities are sparse, the pattern will cover a large area to reach the maximum number of conditioning data $(M)$; on the contrary, when the known conductivities are dense, the pattern will cover a small area and only the nearest nodes are used to account for the local variety. In other words, the flexible search neighborhood scheme has similar effect as multiple-grids (Mariethoz et al., 2010b); (b) only hydraulic heads located near the unknown node ( $N$ at most) are considered rather than all the heads over the field, which facilitates to avoid potential spurious correlation between simulated hydraulic conductivities and head observations.

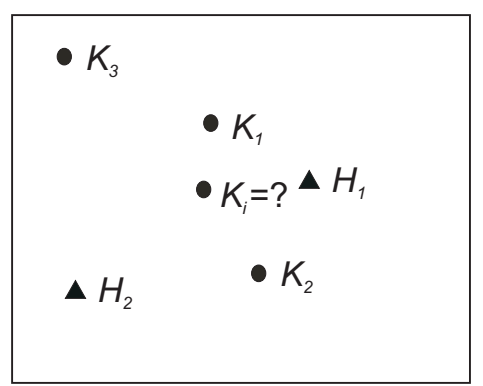

Figure 5.2: A pattern example consisting of conditional hydraulic conductivity and head data. $K_{i}$ is the value to be simulated.

2. Search for a match to the conditional pattern. Randomly start from a realization in the ensemble to search for a match to the conditioning pattern in the ensemble of realizations. Calculate the distance function $(d)$ between the conditioning data and the candidate:

$$
d=\omega d_{k}+(1-\omega) d_{h}
$$

where $d_{k}$ and $d_{h}$ are the distances between the conditioning data and the candidate pattern corresponding to hydraulic conductivities and heads, respectively; $\omega$ is a trade-off coefficient used to balance the influence of the two types of conditioning data. This weight technique has been applied in many inverse methods and a usual choice for the value of $\omega$ is 0.5 when two types of conditioning data are taken into account and the distance measures are normalized (e.g., Alcolea and Renard, 2010; Capilla and LlopisAlbert, 2009; Christiansen et al., 2011; Hendricks Franssen et al., 
2003). Since hydraulic heads depend not only on hydraulic conductivities but also on the boundary conditions and the presence of sinks or sources, the search is performed through the ensemble but only about the position of $K_{i}$. More specifically, in this work, we search only within a 3 by 3 square as shown in Figure 5.3, i.e., only 9 pattern candidates centered in the 3 by 3 square are evaluated in each realization and the matching pattern is expected to be found among them. The expression of the distance function will be discussed later on.

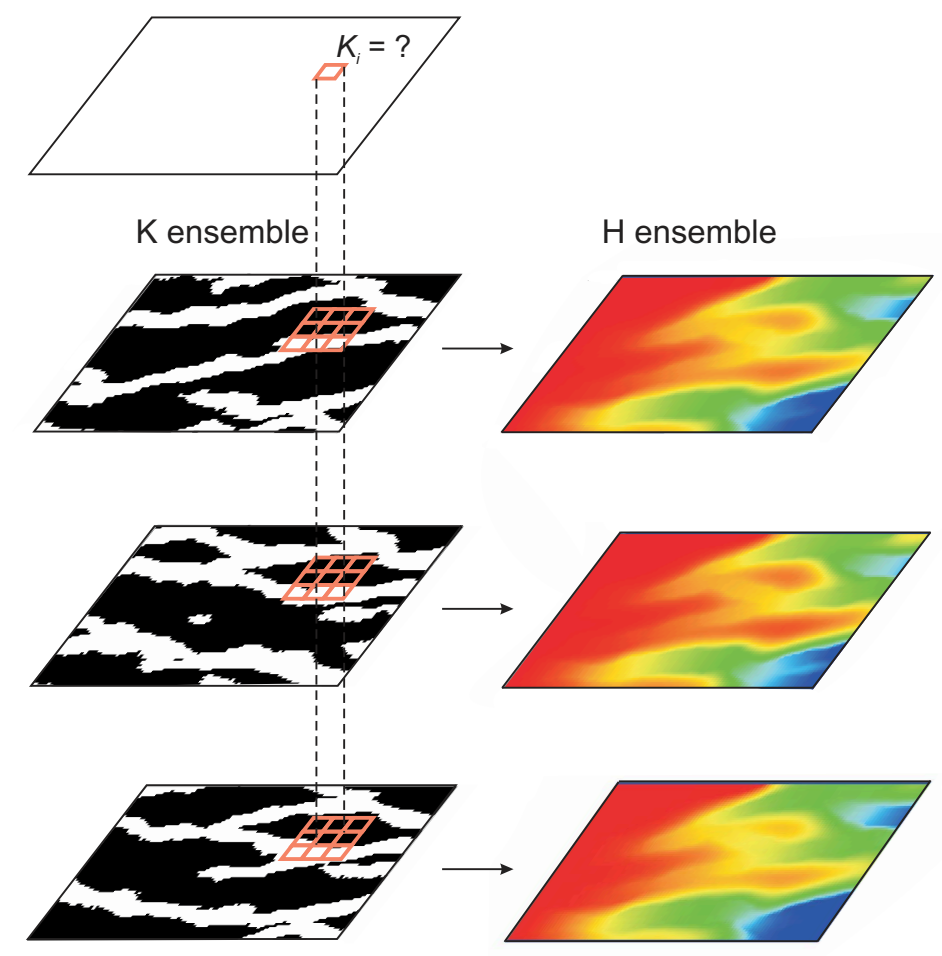

Figure 5.3: Sketch map of the searching strategy. The dashed line indicates the exact location of $K_{i}$ through the ensemble. The candidates in the 3 by 3 square in each realization are evaluated to find the match consistent with the conditioning hydraulic conductivities and observed piezometric heads.

3. Update the $K_{i}$. If the distance function value $d$ is less than a predefined threshold $\left(d_{t}\right)$, extract the value to update $K_{i}$ from the matching pattern and stop searching. If $d_{t}=0$, the conditioning data are exactly matched; if $d_{t}>0$, a certain disagreement is al- 
lowed. To explicitly distinguish the misfits related with hydraulic conductivities and heads in the conditioning data pattern, we can define two thresholds, $d_{t, k}$ and $d_{t, h}$. In the present work, hydraulic conductivities are considered as categorical variables (two facies with uniform values) and the corresponding $d_{t, k}$ is set to 0 , indicating an exact fit. Normally $d_{t, h}$ is assigned a value larger than 0 to account for the measurement errors. If no match is found fulfilling the predefined thresholds, the pattern with the smallest distance value is used.

4. Update the ensemble. Move on to the next cell and then to the next realization until all the grids and realizations in the ensemble are updated.

- Postprocessing. Inconsistencies may appear during data assimilation as shown in Figure 5.4. We can find that the cells indicated by the ellipses are not consistent with their neighboring values, and cannot be considered geologically realistic. We simply filter these inconsistent values out similarly as Henrion et al. (2010) did. However, this might disturb the proportion between facies since no proportion control strategy is applied. In order to reduce the influence of the artificial filtering on facies proportion, we only consider those inconsistent objects consisting of at most three cells. More complex postprocessing methods can be found in image processing algorithms, e.g., kernel principal component analysis (Kim et al., 2005; Mika et al., 1999), and among others (Falivene et al., 2009).

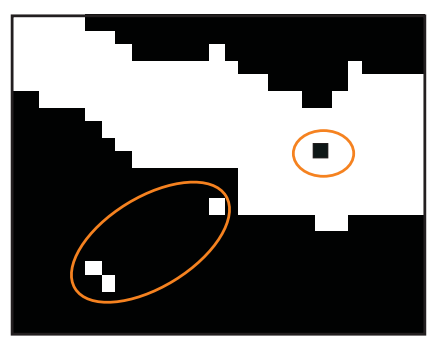

Figure 5.4: Sketch of filtering out noise. The black/white cells are converted to white/black so as to be consistent with the values in the neighborhood.

- Loop back to the forecast step and repeat the process until all observations available are integrated. 


\subsubsection{Distance function}

In the proposed method, the distance function plays a key role and it must be defined carefully. The Minkowski distance is a commonly used distance function as defined below (Borg and Groenen, 2005; Duda et al., 2001).

$$
d\left\{d\left(x_{n}\right), p\left(x_{n}\right)\right\}=\left(\sum_{i=1}^{n}\left|d\left(x_{i}\right)-p\left(x_{i}\right)\right|^{q}\right)^{1 / q} \quad(q \geq 1)
$$

where $d\left\{d\left(x_{n}\right), p\left(x_{n}\right)\right\}$ is the distance function between the data event $d\left(x_{n}\right)$ and the conditioning data pattern $p\left(x_{n}\right), n$ indicates the size of $d\left(x_{n}\right)$ and $p\left(x_{n}\right), x$ can be hydraulic conductivity and head data, and $q$ is a variable that, if equal to 1 , gives rise to the Manhattan distance, and if it is equal to 2 , to the Euclidian distance.

1. Manhattan distance (city-block distance) has been used as the dissimilarity measure in the SIMPAT, a multiple-point geostatistical simulation algorithm (Arpat and Caers, 2007).

- Categorical variables:

$$
\begin{aligned}
& d\left\{d\left(x_{n}\right), p\left(x_{n}\right)\right\}=\frac{1}{n} \sum_{i=1}^{n} a_{i} \quad d \in[0,1] \\
& a_{i}= \begin{cases}0, & \text { if } d\left(x_{i}\right)=p\left(x_{i}\right) \\
1, & \text { otherwise }\end{cases}
\end{aligned}
$$

The distance values are normalized into the range $[0,1]$ by dividing by $n$, which makes it convenient to define the threshold values, i.e., threshold values near 0 indicate very low deviation and near 1 very high deviation. It also helps in combining the distances for different attributes.

- Continuous variables:

$$
d\left\{d\left(x_{n}\right), p\left(x_{n}\right)\right\}=\frac{1}{n} \sum_{i=1}^{n} \frac{\left|d\left(x_{i}\right)-p\left(x_{i}\right)\right|}{d_{\max }} \quad d \in[0,1]
$$

where $d_{\max }$ is the maximum deviation between $d\left(x_{i}\right)$ and $p\left(x_{i}\right)$, together with $n$ used to normalize the distance values.

2. Weighted Euclidean distance attributes different weights to elements in the data event depending on their distance to the simulated node, i.e., the nearer to the simulated node, the more important, while in the unweighted Manhattan distance, all elements share the same weight. 
- Categorical variables:

$$
d\left\{d\left(x_{n}\right), p\left(x_{n}\right)\right\}=\frac{1}{\sum_{i=1}^{n} h_{i}^{-1}} \sum_{i=1}^{n} a_{i} h_{i}^{-1} \quad d \in[0,1]
$$

where $h_{i}$ is the lag distance from the element in the data event to the simulated node and $a_{i}$ is the same as in Equation 5.3.

- Continuous variables:

$$
d\left\{d\left(x_{n}\right), p\left(x_{n}\right)\right\}=\left(\frac{1}{\sum_{i=1}^{n} h_{i}^{-1}} \sum_{i=1}^{n} \frac{\left|d\left(x_{i}\right)-p\left(x_{i}\right)\right|^{2}}{d_{\max }^{2}} h_{i}^{-1}\right)^{1 / 2} d \in[0,1]
$$

where $d_{\max }$ is the same as in Equation 5.4 and $h_{i}$ is the same as in Equation 5.5.

The Manhattan distance and the weighted Euclidian distance functions defined above were first proposed in developing the DS (Mariethoz et al., 2010b) and then modified in this work. Manhattan distance functions (Equations 5.3 and 5.4) are more computationally efficient than Euclidian ones (Equations 5.5 and 5.6). An alternative to the Minkowski-based distance family is the Hausdorff distance (Dubuisson and Jain, 1994), which has been used, for instance, by Suzuki and Caers (2008).

\subsection{A synthetic example}

A synthetic experiment is designed to evaluate the performance of the proposed method. The test aquifer is assumed confined and it covers a domain discretized into $100 \times 80 \times 1$ cells, with cell dimensions of $1 \mathrm{~m} \times 1 \mathrm{~m} \times 10 \mathrm{~m}$. A training image for the facies (Figure 5.5) was generated using the objectbased geologic modeling program FLUVSIM (Deutsch and Tran, 2002). This training image serves as a conceptual model of the bimodal aquifer composed of high permeability sand and low permeability shale. Uniform permeability values are assigned to the two facies, i.e., $\ln K=-4 \mathrm{~m} / \mathrm{d}$ for the shale and $\ln K$ $=1 \mathrm{~m} / \mathrm{d}$ for the sand. DS (Mariethoz et al., 2010b), a pattern-based multiplepoint geostatistical simulation algorithm, is used to generate the reference facies field (Figure 5.6) by borrowing structures from the training image. Hydraulic conductivities at 20 locations in the reference are collected serving as the conditioning hard data (see Figure 5.6 for locations of the measurements).

MODFLOW2000 (Harbaugh et al., 2000), a finite difference flow simulator, is used to solve the transient groundwater flow equation on the reference field subject to the boundary conditions: impermeable boundaries in the north and 


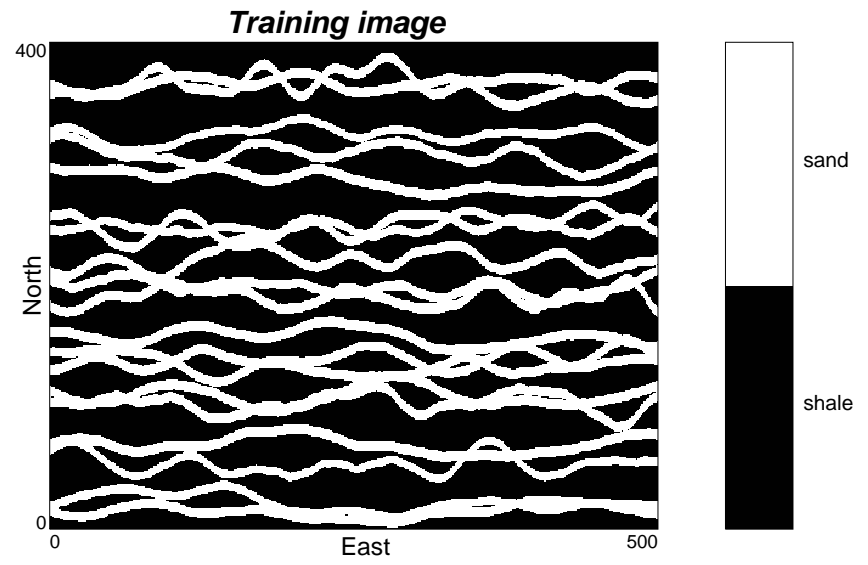

Figure 5.5: Training image used to generate the ensemble of binary facies realizations.

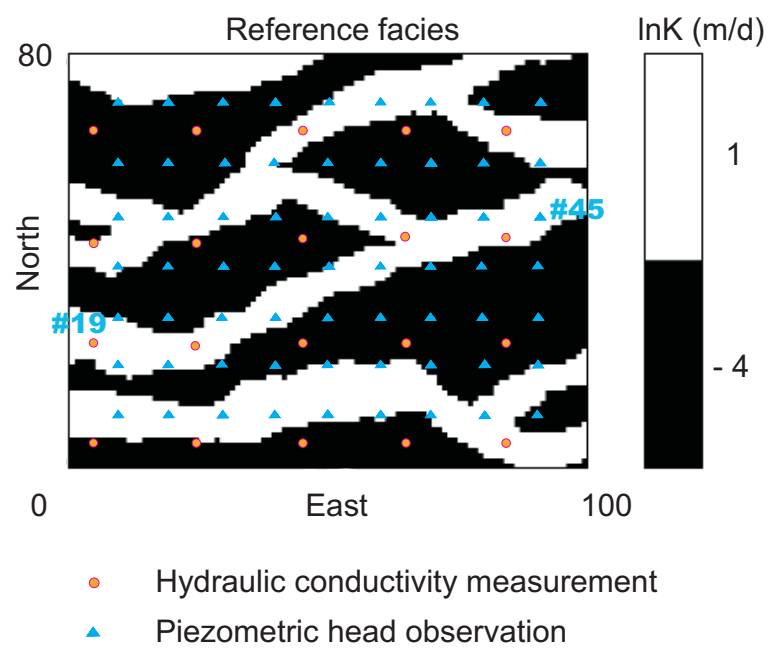

Figure 5.6: Reference facies field. 
south, constant head in the west $(\mathrm{H}=0 \mathrm{~m})$ and prescribed flow rate in the east $\left(\mathrm{Q}=100 \mathrm{~m}^{3} / \mathrm{d}\right)$. Notice that the flow pumping rates in the east boundaries are not uniform, but proportional to the conductivities at the boundary. The initial head is $0 \mathrm{~m}$ everywhere over the field. A simulation period of 30 days is discretized into 20 time steps following a geometric sequence of ratio 1.05. Specific storage is assumed constant and equal to $0.003 \mathrm{~m}^{-1}$. Piezometric head data at 63 observation locations are collected serving as the conditioning data to update the prior model parameters. Configuration of the 63 piezometers is shown in Figure 5.6.

The prior ensemble of realizations consists of 500 realizations which are generated by DS using the same training image used to generate the reference. The 20 conductivity hard data are honored when the prior realizations are generated. The prior ensemble is so generated that the uncertainties related with the conceptual model and hydraulic conductivity measurement are not considered in this experiment.

The observed piezometric heads in the first 6 time steps (6.17 days) are used to update the prior realizations with the proposed method. The results after integrating the observations are presented and discussed in the following section.

\subsection{Results and discussions}

\subsubsection{Hydraulic conductivity characterization}

Figure 5.7 shows the first four realizations in the ensemble before and after the head data are assimilated. The prior realizations (left column) are conditioned to 20 hydraulic conductivity measurements and the updated realizations (right column) are consistent with both measured conductivity and observed piezometric head data. We can find that the prior realizations deviate considerably from the reference field while the updated realizations resemble closely the reference. In other words, the main channel pattern is captured by integrating the observed piezometric heads. However, we notice that the updated realizations exhibit a little higher variability near the west boundaries than in the east (indicated by the three ellipses in the reference field). This can be attributed partly to the boundary conditions, since piezometric heads around prescribed head boundaries are not sensitive to hydraulic conductivity fluctuations.

Figure 5.8 summarizes the prior and posterior statistic metrics of $\ln K$ over the ensemble of realizations. The ensemble average (the second row of Figure 5.8, "EA") of the prior realizations exhibits no channel trend while the updated EA shows clear channels and resembles the reference field. The ensemble standard deviation (the third row of Figure 5.8, "Std. dev.") shows a significant reduction of uncertainty, i.e., in the prior model the uncertain- 


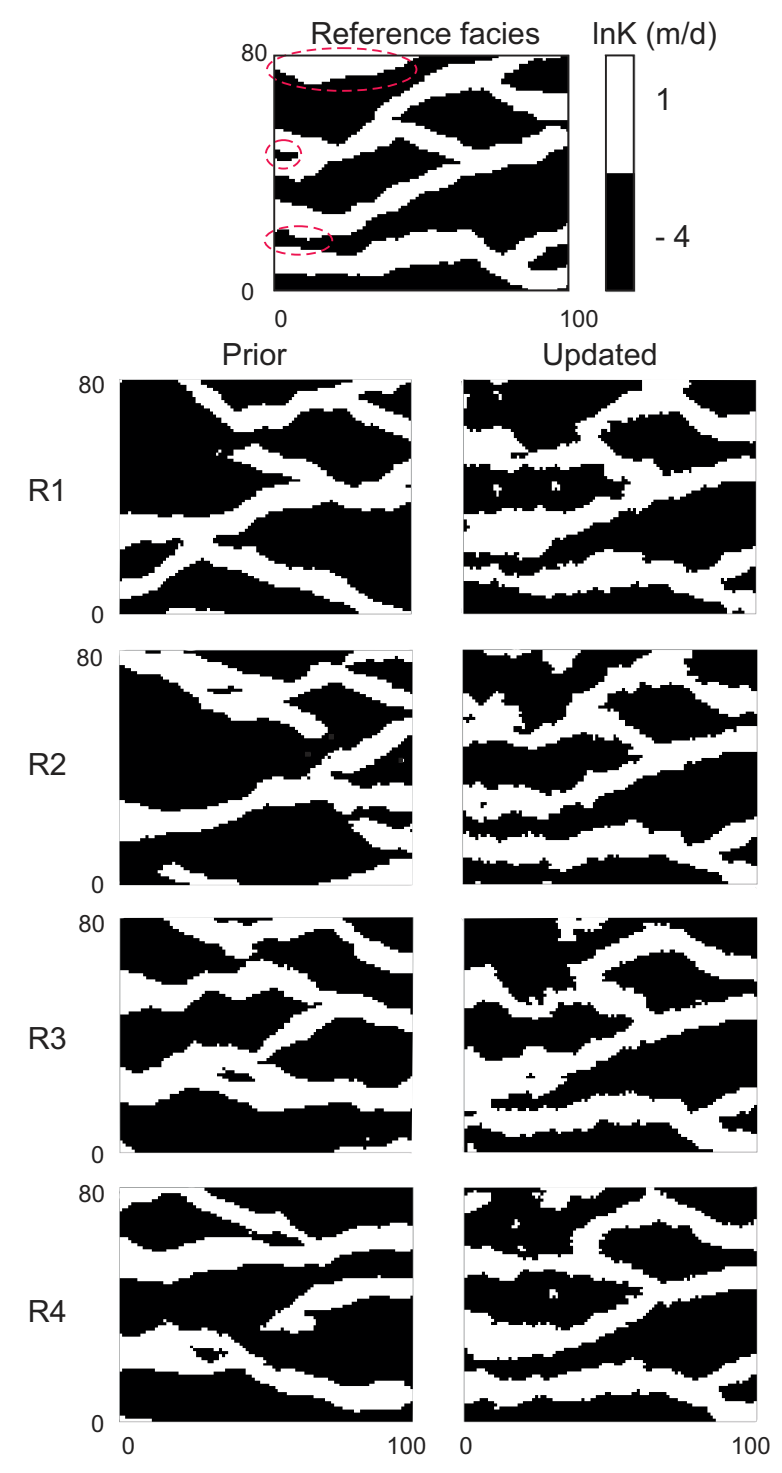

Figure 5.7: The first four realizations in the ensemble. The left column shows four prior facies fields and the right column shows the corresponding updated facies. The reference field is also shown for comparison. 
ties around the hard data are small and the uncertainties grow big when far away from the hard data locations while in the updated case they are reduced everywhere. We also plot the RMSE (the bottom row of Figure 5.8) taking advantage of knowing the reference field exactly. The $R M S E(x)_{i}$ at a node $i$ is computed as

$$
\operatorname{RMSE}(x)_{i}=\left[\frac{1}{N_{r}} \sum_{j=1}^{N_{r}}\left(x_{i, j}^{u p d}-x_{i}^{r e f}\right)^{2}\right]^{1 / 2}
$$

where $N_{r}$ is the number of realizations in the ensemble, $x$ can be either the updated $\ln K$ or the predicted head $h$, the superscripts upd and ref indicate updated and reference model, respectively. Similarly with the standard deviation, the $R M S E(\ln K)$ field confirms the importance of assimilating observed piezometric head data in characterizing the structure of hydraulic conductivity. The error is clearly reduced in the updated ensemble compared with the prior case. Moreover, we calculate the average $R M S E(\ln K)$ over the field and it is reduced from $3.0 \mathrm{~m} / \mathrm{d}$ of the prior model to $1.5 \mathrm{~m} / \mathrm{d}$ of the updated model. As we have mentioned previously, the structure identification near the west boundaries is less improved compared with the east part (separated by the dashed line) due to the influence of the prescribed head boundaries.

\subsubsection{Prediction capability of the updated model}

To evaluate the prediction capacity of the updated model, we will use it to forecast piezometric head evolution and mass transport. The initial and boundary conditions remain the same as during the model calibration. Figure 5.9 shows the evolution of hydraulic head with time in the simulation period (30 days) at two of the piezometers, where the left column shows predictions with prior model and the right column shows predictions with the updated model after assimilating the observed hydraulic head data until 6.17 days. The prediction uncertainty is substantially reduced in the updated $\ln K$ model compared with the prior model. The average $R M S E(h)$ at each time step over the hydraulic field is calculated as shown in Figure 5.10. We can argue that the hydraulic head prediction with the updated model is improved not only at the observation locations but over the whole field. Figure 5.11 summarizes the ensemble average, standard deviation and RMSE of the flow prediction at the end of the simulation with the prior and calibrated model, separatedly.

Figure 5.12 illustrates the configuration of the transport prediction experiment. Conservative particles are released linearly along $x=10 \mathrm{~m}$ and three control planes across the field are placed to record the arrival times of the particles. The random walk particle tracking program RW3D (Fernàndez-Garcia et al., 2005; Salamon et al., 2006; Li et al., 2011a) is used to solve the transport 


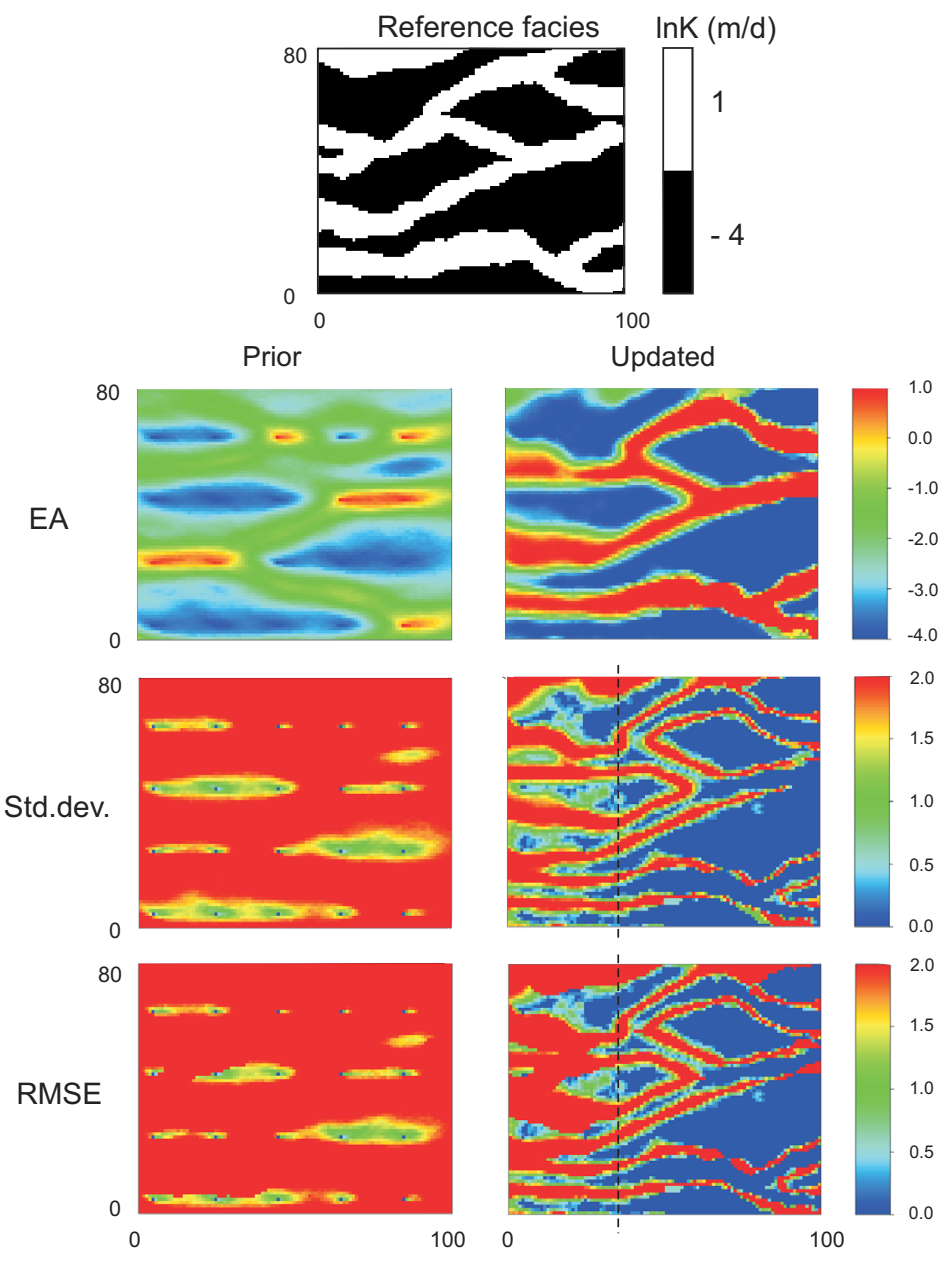

Figure 5.8: Ensemble average (the second row), standard deviation (the third row) and RMSE (the bottom row) of $\ln K$ over the ensemble before and after head data assimilation. The reference field (the top row) is also shown for comparison. 

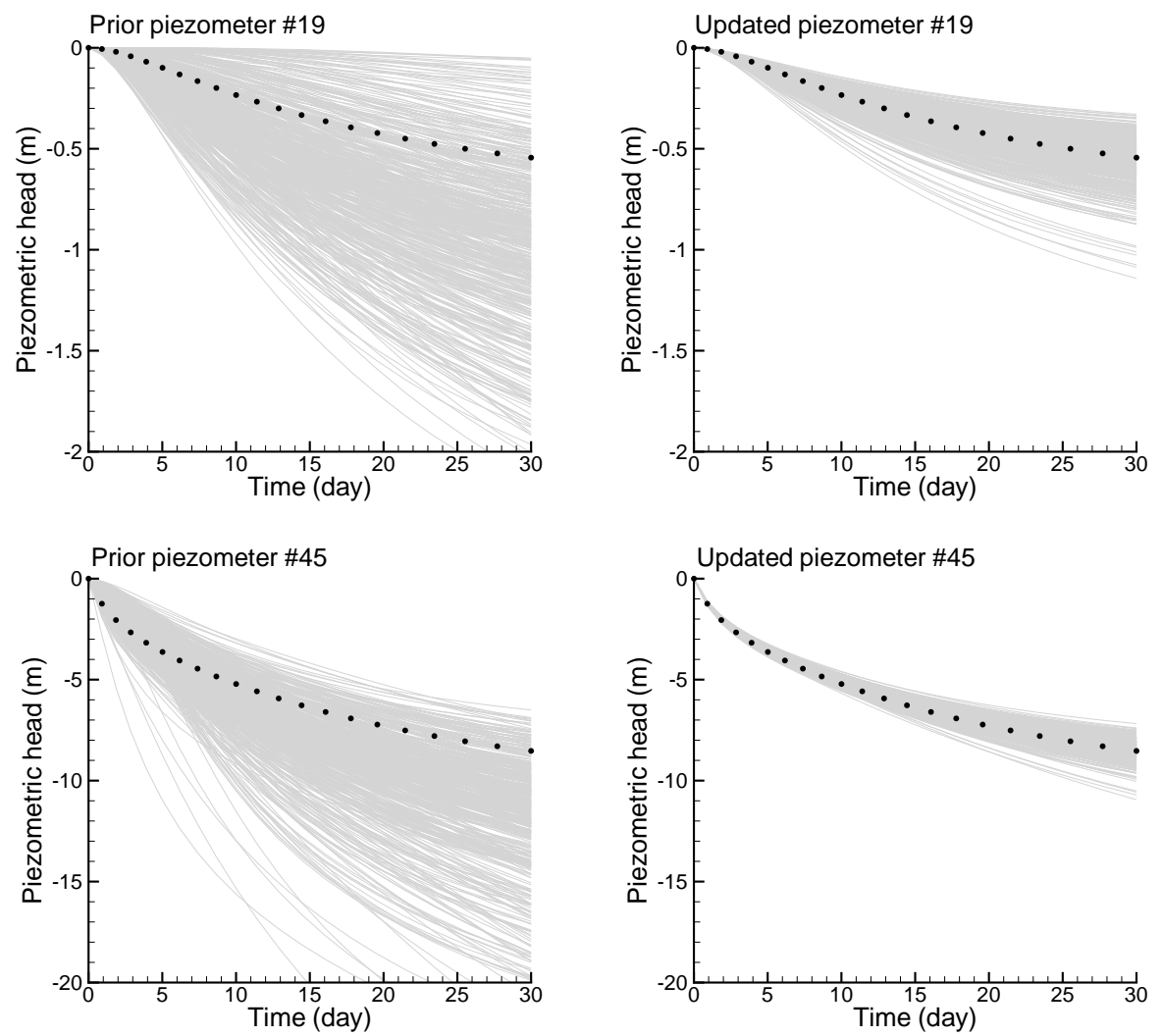

Figure 5.9: Piezometric head evolution at two conditioning piezometers, positions of which are shown in Figure 5.6. Results are shown for the prior ensemble and the updated ensemble. The dots represent the piezometric head in the reference field.

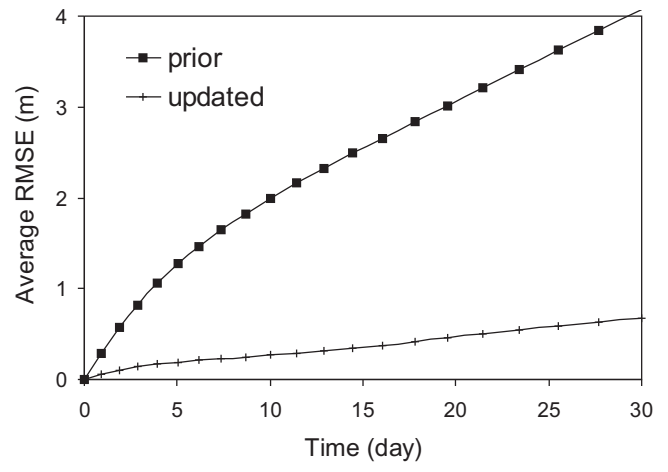

Figure 5.10: Evolution of average $R M S E$ of piezometric heads over the field. 


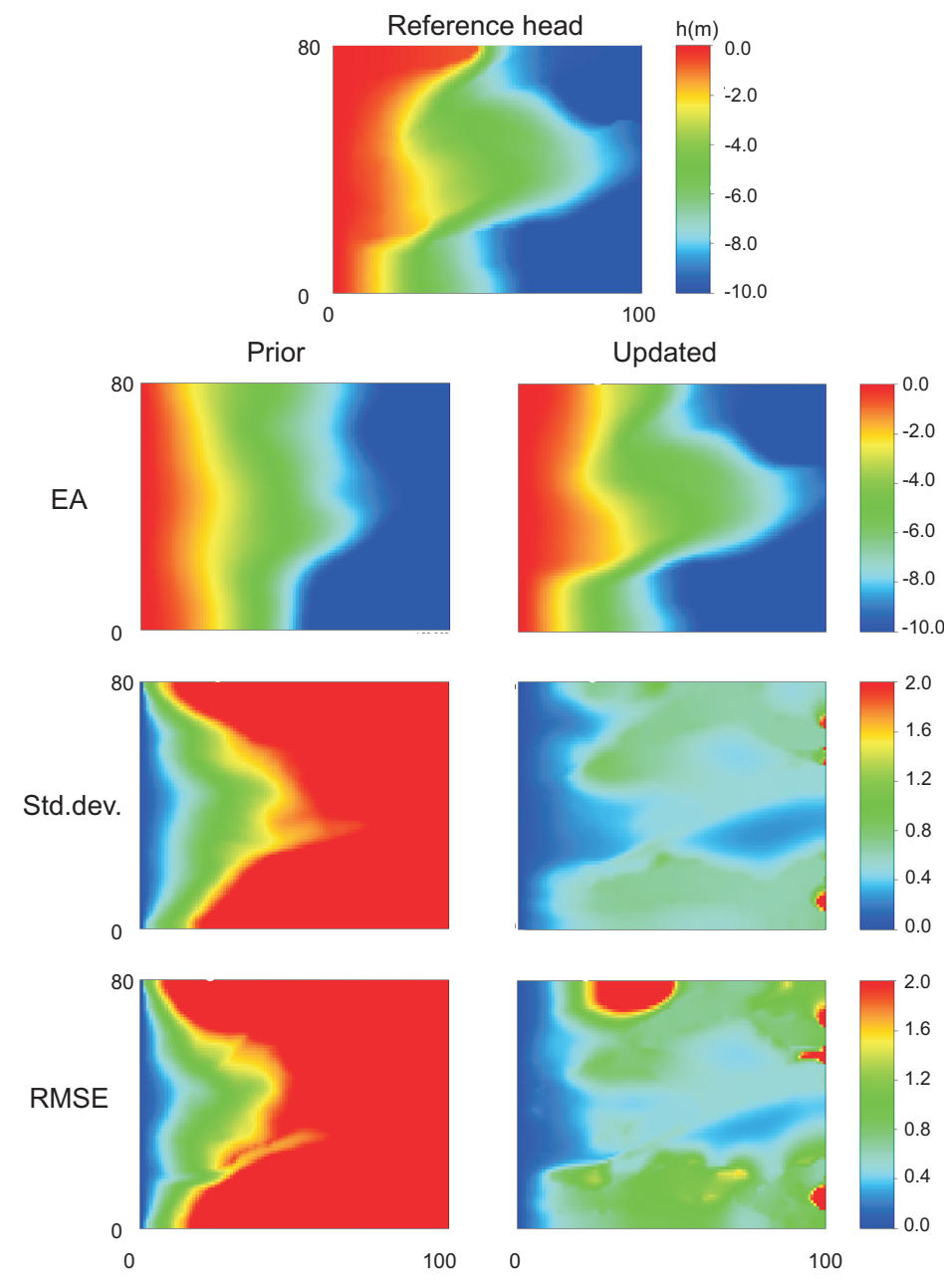

Figure 5.11: Ensemble average (the second row), Standard deviation (the third row) and RMSE (the bottom row) of hydraulic head over the ensemble before and after head data assimilation. Reference head field (the top row) is also shown for comparison. 
equation in the $\ln K$ fields once the flow has reached steady state. Advection and dispersion are both considered, with longitudinal and transverse dispersivities of $0.5 \mathrm{~m}$ and $0.05 \mathrm{~m}$, respectively. The porosity is assumed constant as 0.3. Figure 5.13 shows the breakthrough curves (BTCs) at the three planes for the prior ensemble (left column) and for the updated ensemble (right column). We can see that the updated model reproduces the reference BTCs better than the prior model does, i.e., the median of the travel times in the updated model resembles the reference BTCs. Moreover, the prediction uncertainties measured by the $5^{\text {th }}$ and $95^{\text {th }}$ percentiles are significantly reduced, i.e., the confidence interval is narrower, after the hydraulic heads are conditioned.

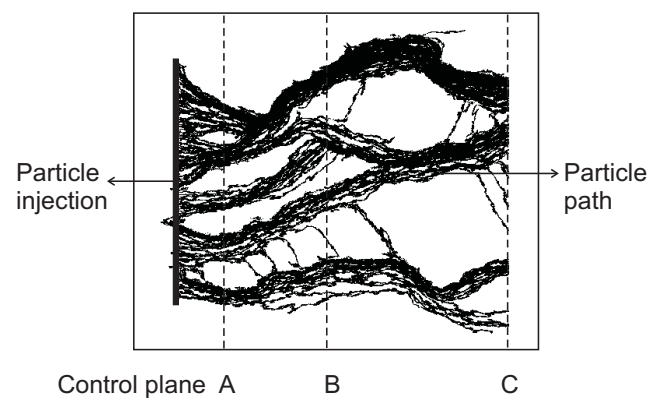

Figure 5.12: Configuration of the transport prediction experiment.

\subsection{A comparison test}

\subsubsection{Reference}

In the previous synthetic example there are 20 hard conductivity data and 63 piezometers used to calibrate the prior model. To further examine the performance of the proposed method we test another application where the observations are available at only 9 locations. Moreover, the effect of boundaries is also considered, i.e., a pumping well is added in this field. This example is similar to the one in Alcolea and Renard (2010) from the respective of the number of conditioning hard data, the number of hydraulic head piezometers, boundary conditions etc.

The research domain of $100 \mathrm{~m} \times 100 \mathrm{~m} \times 10 \mathrm{~m}$ is discretized into $100 \times 100$ $\times 1$ cells. The reference field is generated with the multiple point geostatistical simulation algorithm SNESIM (Strebelle, 2002) using the training image in Figure 5.14A. The reference field is shown in Figure 5.14B, where the hydraulic conductivities are assumed constant within each facies, i.e., $K=10 \mathrm{~m} / \mathrm{d}$ for sand and $K=10^{-3} \mathrm{~m} / \mathrm{d}$ for shale. The transient flow equation is solved on 

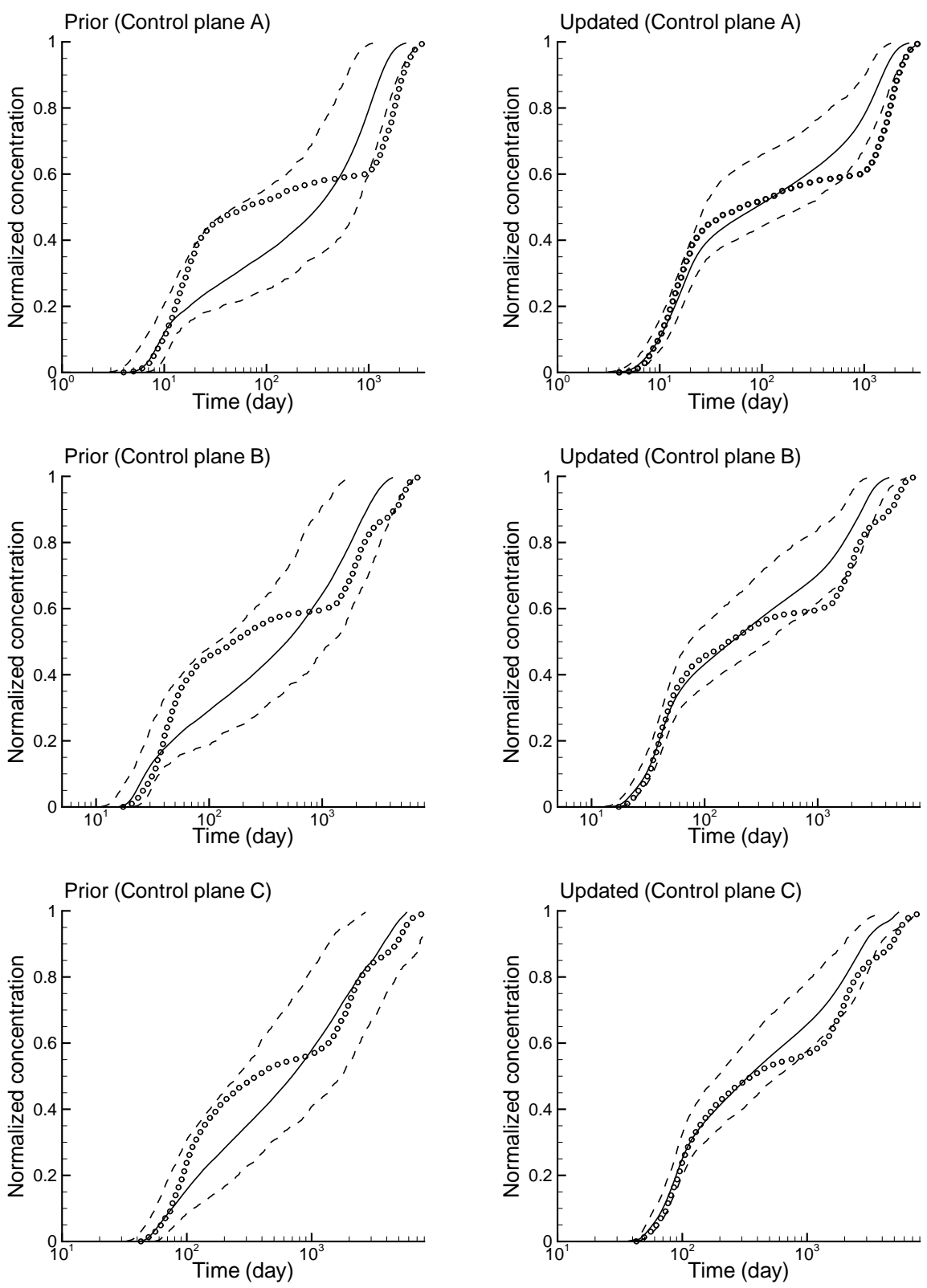

Figure 5.13: Summary of the breakthrough curves. The $5^{\text {th }}$ percentile, the median, and the $95^{\text {th }}$ percentile of the travel times are computed as a function of normalized concentration. Dashed lines correspond to the $5^{\text {th }}$ and $95^{\text {th }}$ percentiles, the solid line corresponds to the median, and the dotted line is the breakthrough curve in the reference. Results are shown for the prior ensemble and the updated ensemble. 
the reference confined aquifer under the boundary conditions: prescribed head boundaries on the west $(\mathrm{H}=1 \mathrm{~m})$ and on the east $(\mathrm{H}=0 \mathrm{~m})$ and impermeable boundaries on the north and south. A pumping well with a production of 100 $\mathrm{m}^{3} / \mathrm{d}$ is located at well 9 in Figure 5.14B. The initial head is $0 \mathrm{~m}$ over the field. The simulation period of 30 days is discretized into 20 time steps following a geometric sequence of ratio 1.2 .
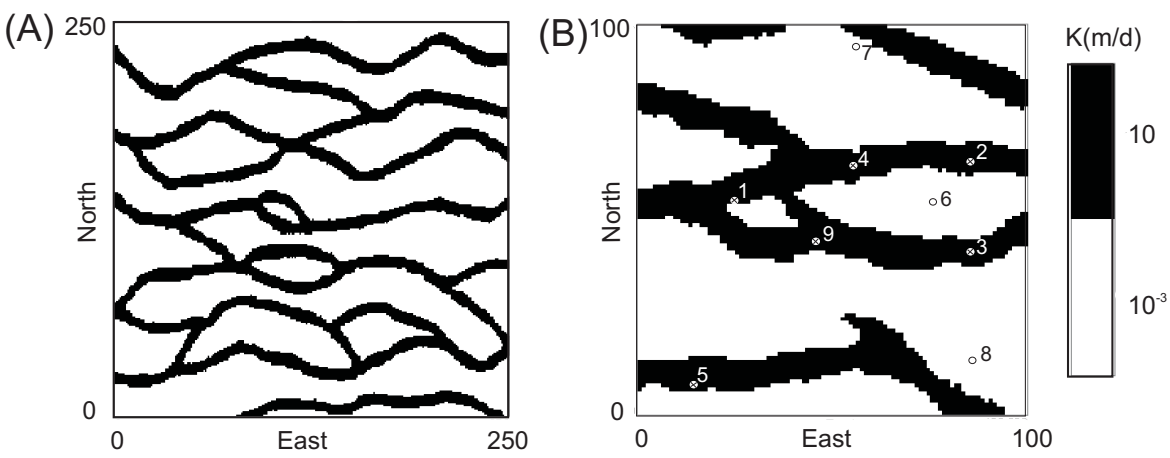

Figure 5.14: Training image and reference field. (A) Training image (Strebelle, 2002). (B) Reference hydraulic conductivity field, in which the conductivities are measured at the 9 points serving as the hard data to generate the prior model and the piezometric head data at these wells are used to calibrate the prior model.

\subsubsection{Prior model and conditioning data}

The prior model ensemble consists of 500 realizations which are generated with the same algorithm (SNESIM) and the same training image (Figure 5.14A). Each realization is conditioned to the 9 lithofacies measured from the reference field (Figure 5.14B) at the nine wells, 6 of which are in sand the the other 3 are in shale. The head dynamics at the 9 wells in the reference field are collected for the first ten time steps (4.17 days) and used to update the prior model. The updated model will be evaluated from facies recognition and flow prediction capacity.

\subsubsection{Calibrated model}

\section{Facies recognition}

Figure 5.15 summarizes the reproduction of the facies by the conditional realizations. On the first row a single realization is shown. It can be seen how, after updating, the channel location is much closer to the one in the reference, 
the main channel features around the conditioning wells are reproduced; however they fail to match the entire length of the isolated branch towards the bottom of the reference, and the branch on the upper right corner. The second row shows the probability that a given cell is in sand, and the third row, the ensemble variance map. When analyzing these last two maps, it is noticeable the improvement that incorporating the piezometric head data brings to the characterization of the hydraulic conductivity field. It is clear that the characterization is best for the channels which are most affected by the presence of the pumping well. The largest uncertainties after updating are next to the left boundary, again due to the lack of sensitivity of the hydraulic conductivities to the piezometric heads next to prescribed head boundaries.

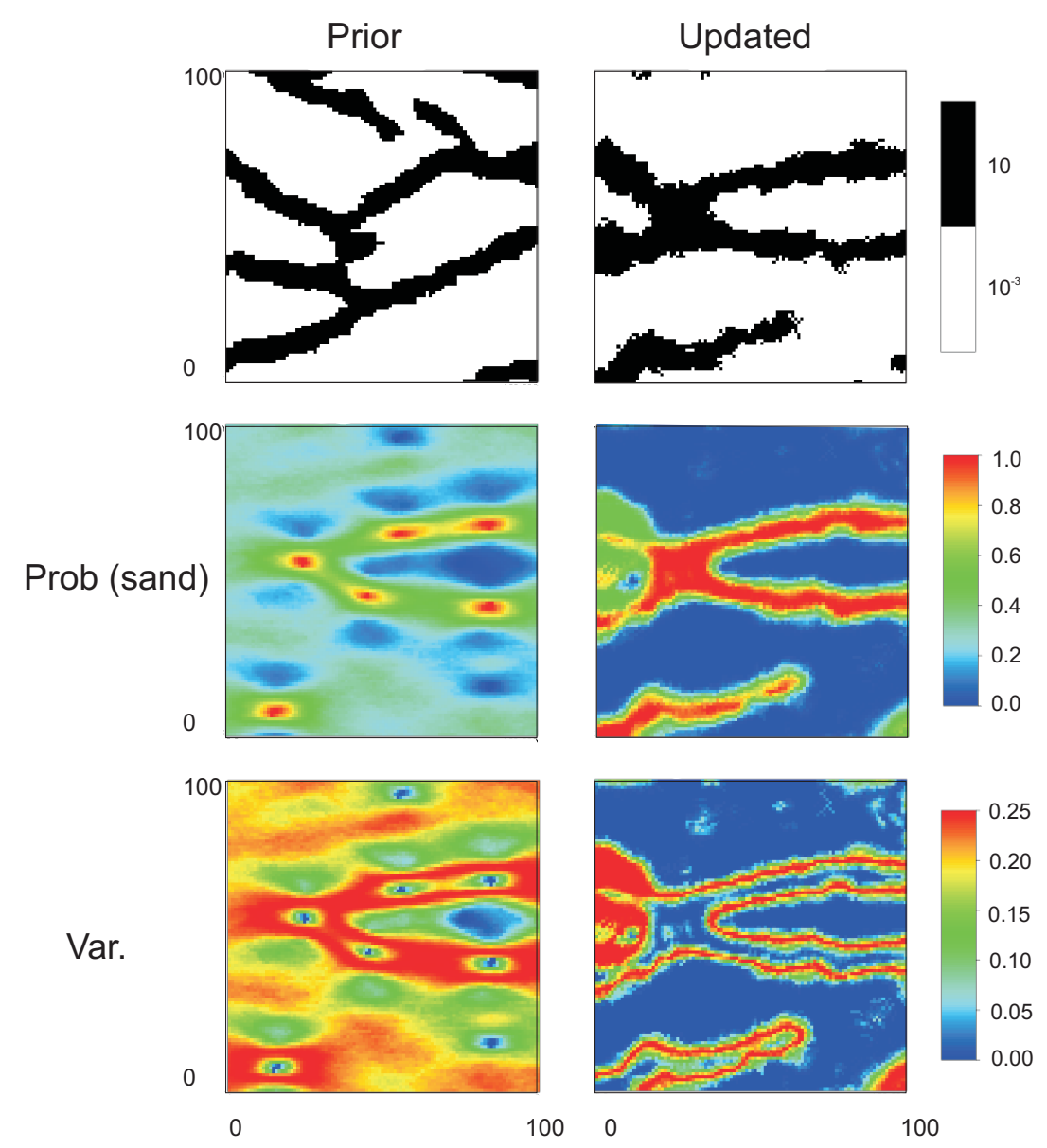

Figure 5.15: Comparison of the prior and calibrated model. A realization of the ensemble (the first row), probability of being sand (the second row) and variance (the third row). 


\section{Flow prediction}

Regarding flow predictions beyond the conditioning period, Figure 5.16 shows the flow prediction at the end of simulation period (30 days) in one realization of the ensemble, and Figure 5.17 displays the head evolution at the 9 wells in the prior and updated model. From Figure 5.16 we can reach similar conclusions as when analyzing the characterization of the conductivities, the updated model does quite a good job except for the part of the channel branch towards the bottom that the conditioning model is not capable of capturing. Figure 5.17 shows the head evolution up to and past the conditioning period in all the 500 realizations before and after updating. We can appreciate the large reduction on the spread of the piezometric head evolution in the different realizations. Analyzing each well individually, we notice that piezometric head assimilation allows setting the barriers that prevent the effect of the pumping to reach wells 7 and 8; well 1 still displays too much fluctuation in the updated model, this is due to the difficulty of the updating algorithm to capture the blob of shale which there is in the reference field between wells 1 and 9 , this failure to capture such a feature may be due to the fact that such a feature is not too recurrent in the training image and therefore it does not replicate often in the 500 realizations; wells 2,3 and 4 are much better reproduced since the main channel branches connecting them to well 9 are there; well 5 evolution is related to its connection to the prescribed head boundary and to the large shale barrier with respect to the pumping well, the reproduction of this two features in the updated fields produces such a good reproduction; well 6 is very well reproduced during the conditioning period, but afterwards the drawdowns are larger than observed, this is indicative that if the conditioning period had been larger, better results would have been obtained; finally, well 9 , the one with the largest drawdowns reduces substantially its fluctuations with regard to the initial realizations, but the conditioning is not as good as in the rest of the wells in absolute terms.

\subsection{Conclusions}

We present a novel inverse method in this paper to estimate model parameters by assimilating the observed flow data. The proposed method aims at recognizing the spatial variety of the nonGaussian-distributed model parameters while guaranteeing the flow responses consistent with the observations. The model parameters are characterized by multiple-point geostatistics which not only relaxes the assumption that the parameters follow a Gaussian distribution but also is able to characterize complex curvilinear geologic structures. The inverse method is based on the Direct Sampling of Mariethoz et al. (2010b) and it is formulated on the basis of pattern searching, i.e., search an 

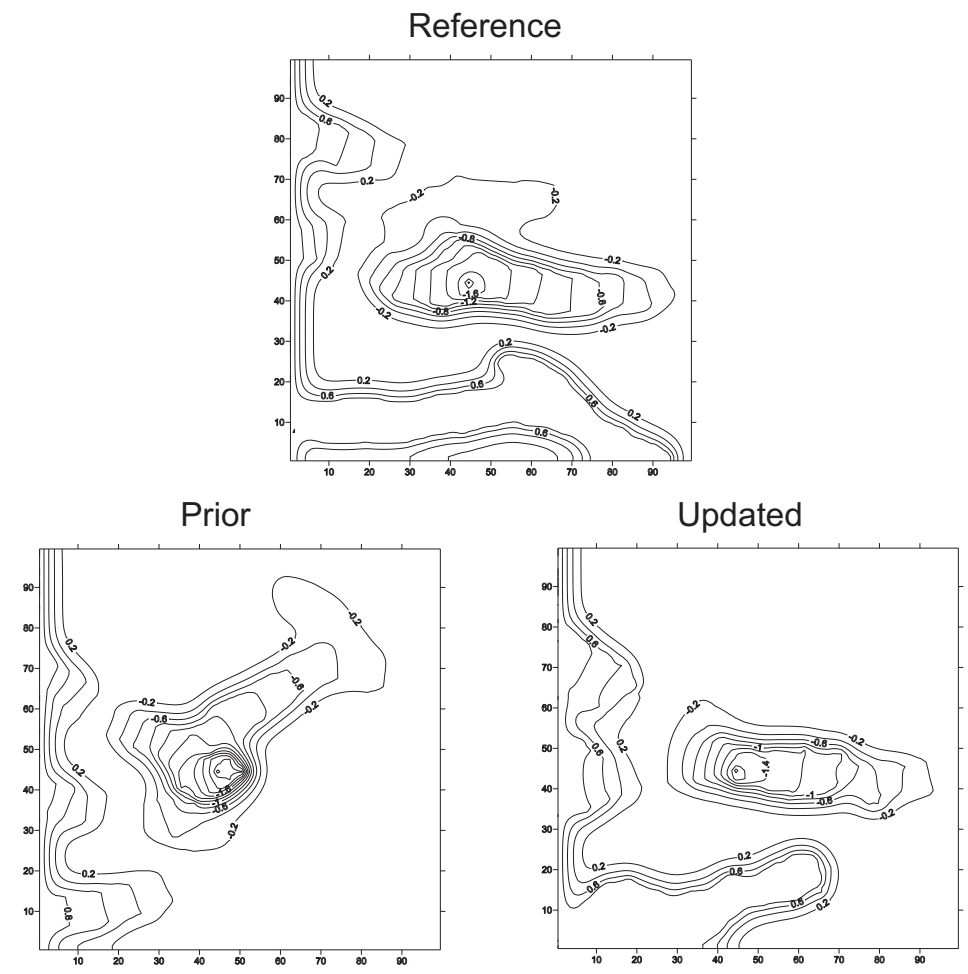

Figure 5.16: Hydraulic head at the end of simulation period in the reference field, prior model and updated model. Only one sample of the realization stack is shown. Hydraulic prediction uncertainty is assessed in the following figure. 

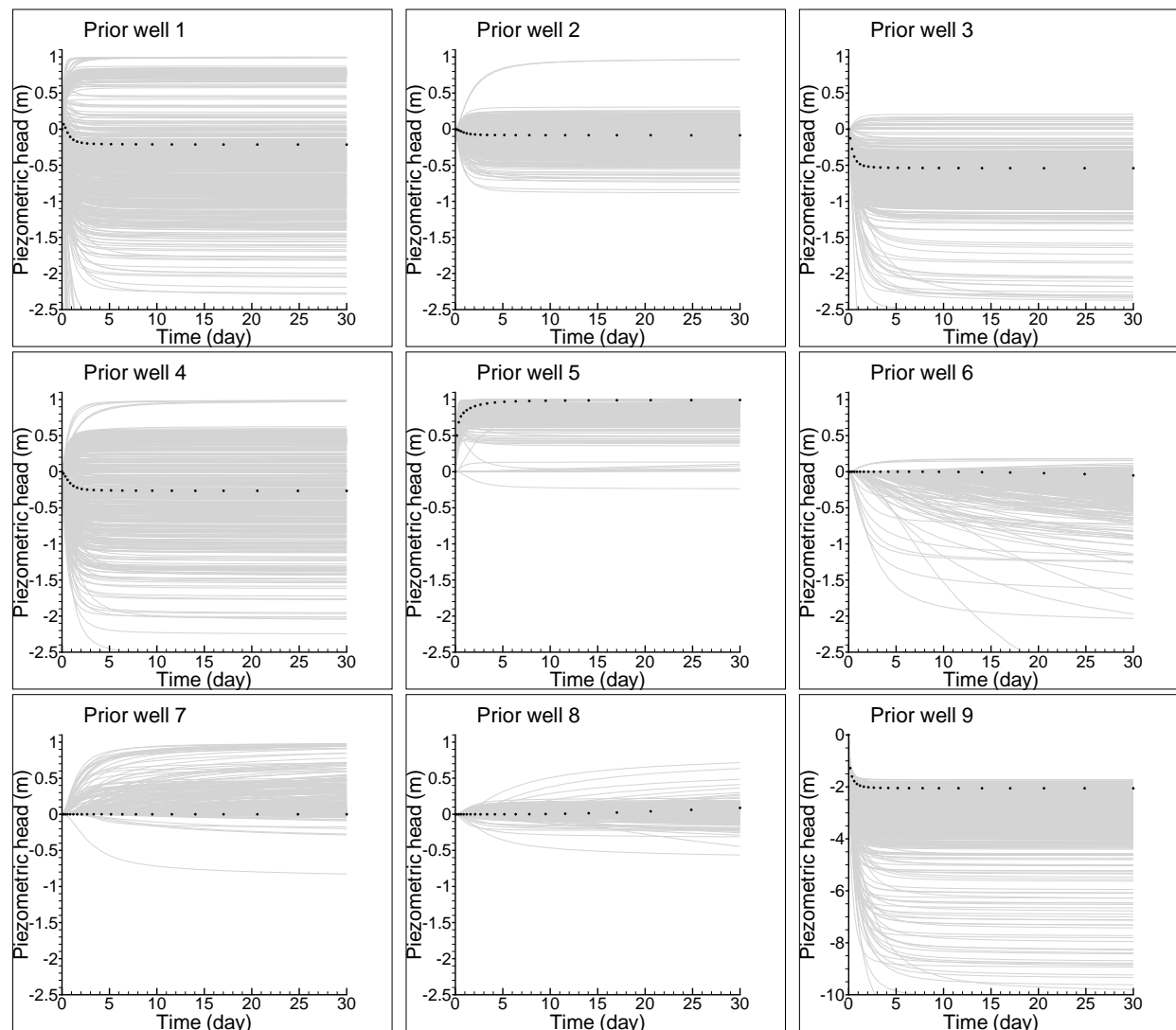

Figure 5.17: Piezometric head evolution at the 9 conditioning piezometers, the positions of which are shown in Figure 5.14B. Results are shown for the prior ensemble (the first 9 plots) and the corresponding updated ensemble (the second 9). The dotted lines represent the piezometric head in the reference. Only the first 6 days were used as conditioning data. (to be continued) 

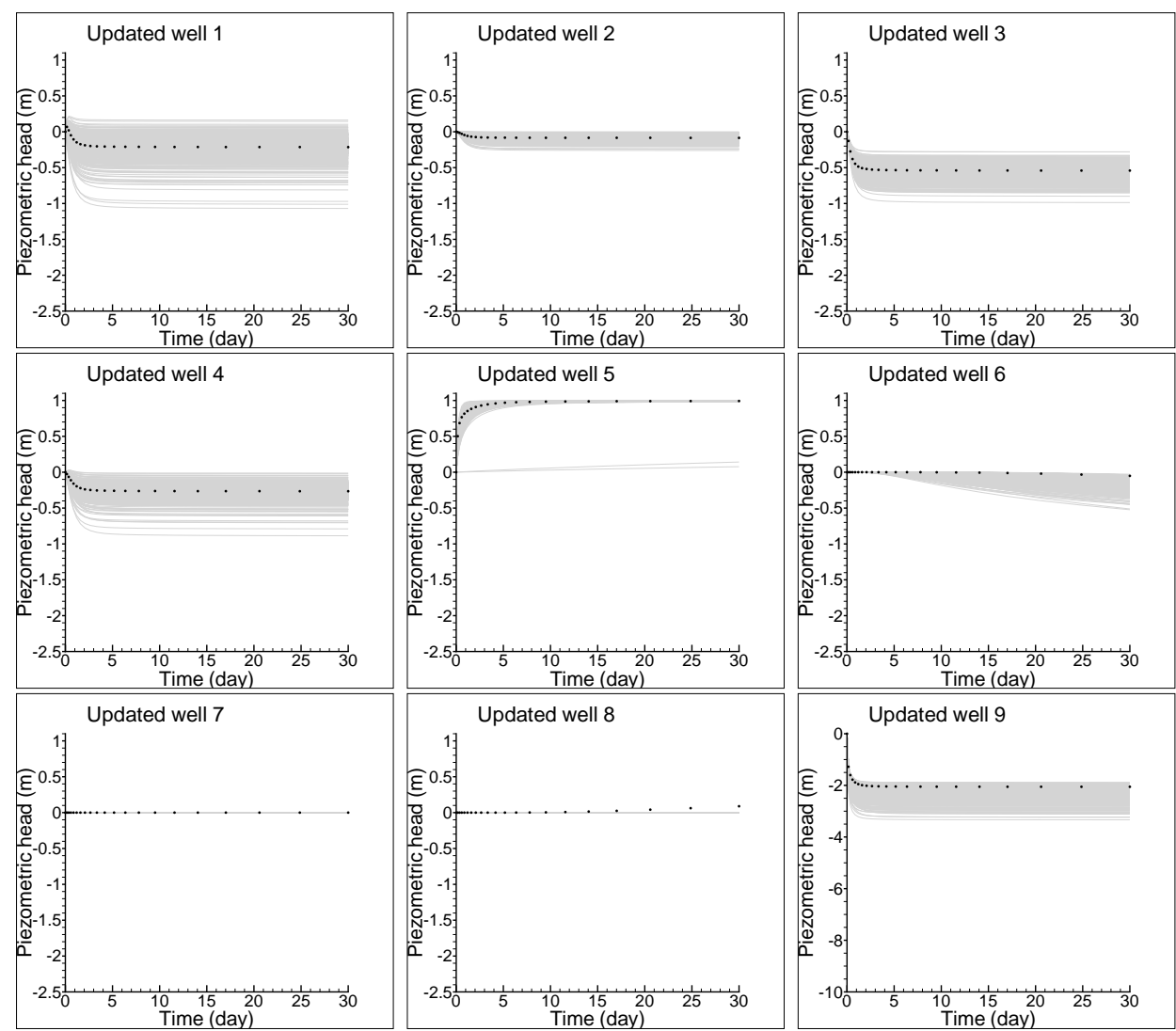
ensemble of realizations for a data set which matches the conditional pattern composed of model parameters and observations. A distance function is introduced to measure the misfit between the conditional pattern and candidates. The searching scheme avoids the need to use any optimization approach, and therefore, the danger of falling onto local minima. Another advantage of the proposed method is that it is not only easy to condition to hard data, since it is a pixel-based method, but it also capable of describing complex geologic features while preserving a prior random function model.

The performance of the proposed method is assessed by two synthetic experiments in an aquifer composed of two facies, sand and shale of contrasting hydraulic conductivity values. The prior hydraulic conductivity models are updated by integrating observed piezometric head data using the proposed method. The main channel structures in the reference field are found to be well reproduced by the updated models. Furthermore, the prediction capacity of the updated models are evaluated in flow and transport simulations, for which both prediction error and uncertainty are significantly reduced.

In the present work, only categorical variables ( $\ln K$ facies) are considered while it is straightforward to extend the method to handle continuous variables. The only difference resides in the scheme of postprocessing. Notice that the proposed method can be time-consuming when a large ensemble is considered, but the CPU time can be reduced by parallelization, i.e., the Monte Carlo simulations can be performed in parallel by more than one processor. 


\section{Bibliography}

Alcolea, A., Renard, P., 2010. Blocking Moving Window algorithm: Conditioning multiple-point simulations to hydrogeological data. Water Resources Research 46 (8), W08511.

Arpat, G. B., Caers, J., 2007. Conditional simulation with patterns. Mathematical Geology 39 (2), 177-203.

Borg, I., Groenen, P. J., 2005. Modern multi-dimensioal scaling: Theory and application, 2nd edn. Springer., New York.

Caers, J., 2002. Geostatistical history matching under training-image based geological model constraints. SPE Annual Technical Conference and Exhibition, San Antonio, Texas, SPE 77429.

Caers, J., 2003. Efficient gradual deformation using a streamline-based proxy method. Journal of Petroleum Science and Engineering 39 (1-2), 57-83.

Caers, J., Hoffman, T., 2006. The probability perturbation method: A new look at bayesian inverse modeling. Mathematical Geology 38 (1), 81-100.

Capilla, J., Llopis-Albert, C., 2009. Gradual conditioning of non-Gaussian transmissivity fields to flow and mass transport data: 1 . Theory. Journal of Hydrology 371 (1-4), 66-74.

Carrera, J., Neuman, S. P., 1986. Estimation of aquifer parameters under transient and steady state conditions: 1. Maximum likelihood method incorporating prior information. Water Resources Research 22 (2), 199-210.

Chen, Y., Zhang, D., 2006. Data assimilation for transient flow in geologic formations via ensemble Kalman filter. Advances in Water Resources 29 (8), $1107-1122$.

Christiansen, L., Binning, P. J., Rosbjerg, D., Andersen, O. B., BauerGottwein, P., 2011. Using time-lapse gravity for groundwater model calibration: An application to alluvial aquifer storage. Water Resources Research, doi: $10.1029 / 2010$ WR009859. 
De Marsily, G., Delay, F., Gonçalvès, J., Renard, P., Teles, V., Violette, S., 2005. Dealing with spatial heterogeneity. Hydrogeology Journal 13 (1), 161183.

Deutsch, C., Tran, T., 2002. FLUVSIM: a program for object-based stochastic modeling of fluvial depositional systems. Computers \& Geosciences 28 (4), $525-535$.

Dimitrakopoulos, R., Mustapha, H., Gloaguen, E., 2010. High-order Statistics of Spatial Random Fields: Exploring Spatial Cumulants for Modeling Complex Non-Gaussian and Non-linear Phenomena. Mathematical Geosciences $42(1), 65-99$.

Dubuisson, M.-P., Jain, A. K., 1994. A modified Hausdorff distance for object matching. In: International conference on pattern recognition. Jerusalem, Israel, pp. 566-568.

Duda, R. O., Hart, P. E., Stork, D. G., 2001. Pattern classification, 2nd edn. John Wiley \& Sons.

Emsellem, Y., De Marsily, G., 1971. An automatic solution for the inverse problem. Water Resources Research 7 (5), 1264-1283.

Evensen, G., 2003. The Ensemble Kalman Filter: Theoretical formulation and practical implementation. Ocean dynamics 53 (4), 343-367.

Falivene, O., Cabello, P., Arbués, P., Muñoz, J. A., Cabrera, L., 2009. A geostatistical algorithm to reproduce lateral gradual facies transitions: Description and implementation. Computers \& Geosciences 35 (8), 1642-1651.

Fernàndez-Garcia, D., Illangasekare, T., Rajaram, H., 2005. Differences in the scale dependence of dispersivity and retardation factors estimated from forced-gradient and uniform flow tracer tests in three-dimensional physically and chemically heterogeneous porous media. Water Resources Research 41 (3), W03012.

Feyen, L., Caers, J., 2006. Quantifying geological uncertainty for flow and transport modeling in multi-modal heterogeneous formations. Advances in Water Resources 29 (6), 912-929.

Fu, J., Gómez-Hernández, J. J., 2009. A blocking Markov chain Monte Carlo method for inverse stochastic hydrogeological modeling. Mathematical Geosciences 41 (2), 105-128.

Gómez-Hernández, J. J., Sahuquillo, A., Capilla, J. E., 1997. Stochastic simulation of transmissivity fields conditional to both transmissivity and piezometric data-I. Theory. Journal of Hydrology 203 (1-4), 162-174. 
Guardiano, F., Srivastava, R., 1993. Multivariate geostatistics: beyond bivariate moments. In: Soares, A. (Ed.), Geostatistics-Troia. Kluwer Academic Publ, Dordrecht, pp. 133-144.

Harbaugh, A. W., Banta, E. R., Hill, M. C., McDonald, M. G., 2000. MODFLOW-2000, the U.S. geological survey modular ground-water model - user guide to modularization concepts and the ground-water flow process. Tech. Rep. Open-File Report 00-92, U.S. Department of the Interior, U.S. Geological Survey, Reston, Virginia, 121pp.

Hendricks Franssen, H. J., Gómez-Hernández, J. J., Sahuquillo, A., 2003. Coupled inverse modelling of groundwater flow and mass transport and the worth of concentration data. Journal of Hydrology 281 (4), 281-295.

Hendricks Franssen, H. J., Kinzelbach, W., 2008. Real-time groundwater flow modeling with the Ensemble Kalman Filter: Joint estimation for states and parameters and the filter inbreeding problem. Water Resources Research 44, W09408.

Henrion, V., Caumon, G., Cherpeau, N., 2010. ODSIM: An Object-Distance Simulation Method for Conditioning Complex Natural Structures. Mathematical Geosciences 42 (8), 911-924.

Hu, L. Y., 2000. Gradual deformation and iterative calibration of Gaussianrelated stochastic models. Mathematical Geology 32 (1), 87-108.

Hu, L. Y., Chugunova, T., 2008. Multiple-point geostatistics for modeling subsurface heterogeneity: A comprehensive review. Water Resources Research 44 (11), W11413.

Huysmans, M., Dassargues, A., 2009. Application of multiple-point geostatistics on modelling groundwater flow and transport in a cross-bedded aquifer (Belgium). Hydrogeology Journal 17 (8), 1901-1911.

Jafarpour, B., Khodabakhshi, M., 2011. A probability conditioning method (PCM) for nonlinear flow data integration into multipoint statistical facies simulation. Mathematical Geosciences 43 (2), 133-164.

Journel, A., Zhang, T., 2006. The necessity of a multiple-point prior model. Mathematical geology 38 (5), 591-610.

Kerrou, J., Renard, P., Hendricks Franssen, H. J., Lunati, I., 2008. Issues in characterizing heterogeneity and connectivity in non-multiGaussian media. Advances in Water Resources 31 (1), 147-159. 
Kim, K. I., Franz, M. O., Schölkppf, B., 2005. Iterative kernel principal component analysis for image modeling. IEEE Transactions on Pattern Analysis and Machine Intelligence 27 (9), 1351-1366.

Kitanidis, P. K., 2007. On stochastic inverse modeling. In: Hyndman, D. W., Day-Lewis, F. D., Singha, K. (Eds.), Subsurface hydrology: Data integration for properties and processes. American Geophysical Union, Washington, DC, pp. 19-30.

Li, L., Zhou, H., Gómez-Hernández, J. J., 2011a. Transport upscaling using multi-rate mass transfer in three-dimensional highly heterogeneous porous media. Advances in Water Resources 34 (4), 478-489.

Li, L., Zhou, H., Hendricks Franssen, H. J., Gómez-Hernández, J. J., $2011 b$. Groundwater flow inverse modeling in non-multigaussian media: performance assessment of the normal-score ensemble kalman filter. Hydrology and Earth System Sciences Discussions 8 (4), 6749-6788.

Mariethoz, G., Renard, P., Caers, J., 2010a. Bayesian inverse problem and optimization with iterative spatial resampling. Water Resources Research 46 (11), W11530.

Mariethoz, G., Renard, P., Straubhaar, J., 2010b. The direct sampling method to perform multiple-point geostatistical simulaitons. Water Resources Research 46, W11536.

Mika, S., Schölkopf, B., Smola, A. J., Müller, K. R., Scholz, M., Rätsch, G., 1999. Kernel PCA and de-noising in feature spaces. Advances in neural information processing systems 11 (1), 536-542.

Mustapha, H., Dimitrakopoulos, R., 2010. High-order stochastic simulations for complex non-Gaussian and non-linear geological patterns. Mathematical Geosciences 42 (5), 455-473.

Neuman, S. P., 1973. Calibration of distributed parameter groundwater flow models viewed as a multiple-objective decision process under uncertainty. Water Resources Research 9 (4), 1006-1021.

Oliver, D. S., Cunha, L. B., Reynolds, A. C., 1997. Markov chain Monte Carlo methods for conditioning a permeability field to pressure data. Mathematical Geology 29 (1), 61-91.

RamaRao, B., LaVenue, A., De Marsily, G., Marietta, M., 1995. Pilot point methodology for automated calibration of an ensemble of conditionally simulated transmissivity fields 1 . Theory and computational experiments. Water Resources Research 31 (3), 475-493. 
Rubin, Y., Chen, X., Murakami, H., Hahn, M., 2010. A Bayesian approach for inverse modeling, data assimilation, and conditional simulation of spatial random fields. Water Resources Research 46, W10523.

Salamon, P., Fernàndez-Garcia, D., Gómez-Hernández, J., 2006. A review and numerical assessment of the random walk particle tracking method. Journal of Contaminant Hydrology 87 (3-4), 277-305.

Strebelle, S., 2002. Conditional simulation of complex geological structures using multiple-point statistics. Mathematical Geology 34 (1), 1-21.

Suzuki, S., Caers, J., 2008. A distance-based prior model parameterization for constraining solutions of spatial inverse problems. Mathematical Geosciences 40 (4), 445-469.

Wen, X. H., Capilla, J. E., Deutsch, C. V., Gómez-Hernández, J. J., Cullick, A. S., 1999. A program to create permeability fields that honor single-phase flow rate and pressure data. Computers \& Geosciences 25 (3), 217-230.

Zhang, T., Switzer, P., Journel, A., 2006. Filter-based classification of training image patterns for spatial simulation. Mathematical geology 38 (1), 63-80.

Zhou, H., Gómez-Hernández, J. J., Hendricks Franssen, H.-J., Li, L., 2011. An approach to handling Non-Gaussianity of parameters and state variables in ensemble Kalman filter. Advances in Water Resources 34 (7), 844-864. 


\section{6 \\ General Conclusions}

\subsection{Summary}

The main point of this thesis is proposing inverse methods to characterize model parameters which cannot be characterized by Gaussian distribution in complex aquifers. Two solutions are considered in the work, i.e., the normalscore ensemble Kalman filter (NS-EnKF) and a pattern searching-based inverse method (PSINV).

The standard EnKF is known to perform optimally when the model parameters and state variables follow a multiGaussian distribution. To cope with the nonGaussian distribution, the standard EnKF is modified by normal-score transforming the state vector so that each component follows a marginal Gaussian distribution. The nonGaussian distributed model vector is transformed into the Gaussian space with the help of an experimental conditional distribution function which is constructed based on the ensemble realizations. Then, the standard EnKF is applied on the transformed state vector and finally the resulting updated vector is backtransformed to the original space. The NSEnKF therefore can be used to characterize nonGaussian distributed model parameters by integrating observed model responses. Furthermore, the prior model statistics are preserved since the normal score transformation scheme is built on the basis of a prior ensemble. In this thesis the NS-EnKF is used to identify the hydraulic conductivities in a synthetic bimodal aquifer conditioning to the observed piezometric head data. The proposed method performs 
better than the standard EnKF in recognizing the channel structure of the subsurface reservoir and for flow and transport predictions.

Sensitivity of the NS-EnKF to different parameters is analyzed, i.e., time span of data assimilation, number of conditioning hard data, size of the ensemble and variance. The method is found to not be very sensitive to the number of conditioning hard data in that the underlying model structure is identified even without any hydraulic conductivity measurements. Notice that more piezometers are needed whenever no hard data available. The impact of variance magnitude is not serious since the method performs well in the case with log-conductivity close to 10 . The size of the ensemble, i.e., number of realizations, exerts strong influence on the method since good results are obtained with 1000 realizations while the results deteriorate when only 200 ones are used.

In contrast with the NS-EnKF, a pattern search-based inverse method, PSINV, is proposed to estimate model parameters by assimilating observations. The PSINV is formulated in the framework of pattern search basis rather than optimization of an objective function. The joint relation between the model parameters and state variables are characterized by the conditioning pattern around the unknown parameter. In other words, the conditioning data pattern consists of not only the observations of model states but also the simulated parameters. This configuration of the pattern is borrowed from multiple-point geostatistics which is capable of characterizing nonlinear structures. Then the ensemble is searched for a match to the conditioning pattern and the unknown value is replaced by the one whose neighborhood matches the conditioning pattern.

Features of the PSINV can be summarized into four aspects. First, the algorithm is formulated by means of pattern searching and no minimization and sensitivity matrix are involved, which will circumvent the potential local minimum value and also makes it time efficient. Second, the model parameters are not assumed to follow a multiGaussian distribution, i.e., the method is supposed to perform well no matter what distribution the parameters follow. Third, the prior structure is preserved during the observations are assimilated since the candidates for matches are found within the prior ensemble. Finally, multiple-point geostatistics is utilized to characterize the curvilinear structures which cannot be described by conventional two-point variogram based model. Performance of the PSINV is assessed in two synthetic aquifers composed of two facies, sand and shale. 


\subsection{Recommendations for future research}

Several topics deserving further investigation about the two methods are listed as follows:

- Considering jointly multivariate transformations in NS-EnKF. The NSEnKF only uses marginally distributed Gaussian variables, it would be interesting to investigate the possibility of making a transformation that would yield the state vector truly multiGaussian.

- Localization of NS-EnKF. In the NS-EnKF no localization strategy is applied while it is found that filter degeneration may appear when the ensemble size is small and the parameter field is highly heterogeneous as shown in the numerical experiment in Chapter 3. Several localization measures for the standard EnKF can be tried on the NS-EnKF, e.g., Schur product (Houtekamer and Mitchell, 2001) or streamline-based localization methods (Devegowda et al., 2010).

- Further evaluation and improvement of the program PSINV.

i. Sensitivity analysis of the program regarding parameters involved. Evaluate influence of the variables used in the program, e.g., number of conditioning hard data, number of hydraulic head observations, threshold of acceptance in distance function, size of the conditioning pattern and number of realizations in the ensemble. Instructions on assigning the parameter values can be provided based on the sensitivity analysis.

ii. Parallelization of the code. The PSINV can be time consuming when a large number of samples are considered. One solution is to distribute the task to more than one processor, i.e., parallelizing the code. Parallelization can be carried out on two parts of the program, i.e., forecast of the state variables and update of the prior parameters. It should not be very challenging to parallelize the code since the parameter realizations in the ensemble are updated one by one in the current code.

iii. Extension to deal with continuous variables. The present code can only handle discrete model parameters, e.g., facies variables, and some work has to be done before the program can cope with continuous parameters. It is indeed straightforward to extend the code to deal with continuous variables and only two problems deserve considering, i.e., distance function and postprocessing, in which the former is already prepared for the continuous variables and the latter needs more investigation. 
iv. Generate new realizations whenever no match is found. New realizations can be generated during an iteration whenever no pattern match is found and at the same time to prevent the likely underestimation of uncertainty.

- Conditioning to multiple sources of information. The integrated data in this work consist of measured hard data and dynamic hydraulic heads. Plentiful observations such as solute concentration and remote sensing data can also be integrated by the two inverse methods.

- Extension to estimate other model properties. Hydraulic conductivity is assumed the only uncertain model parameter in the experiments involved in the thesis, while other parameters, such as porosity and specific storage, may also need recognizing in practice. It is thus necessary to jointly estimate these model parameters with the inverse methods. The standard EnKF has been used to estimate several types of unknown parameters and this avenue can be introduced to the NS-EnKF. With regard to PSINV, the work is to add one more set ensemble of realizations.

- Comparison with other inverse methods and application in the field. The proposed two methods are evaluated in synthetic experiments and they are found to perform well in these examples. However, these numerical examples are specific and synthetic. The performance of the methods should be reinforced by comparing with other inverse approaches and by applying to the real data from the field. 


\section{Bibliography}

Arulampalam, M. S., Maskell, S., Gordon, N., Clapp, T., 2002. A tutorial on particle filters for online nonlinear/non-Gaussian Bayesian tracking. IEEE Transactions on signal processing 50 (2), 174-188.

Deutsch, C. V., Journel, A. G., 1998. GSLIB, Geostatistical Software Library and User's Guide, 2nd Edition. Oxford University Press, New York, 384pp.

Devegowda, D., Arroyo-Negrete, E., Datta-Gupta, A., 2010. Flow relevant covariance localization during dynamic data assimilation using EnKF. Advances in Water Resources 33, 129-145.

Gómez-Hernández, J. J., Wen, X. H., 1998. To be or not to be multi-Gaussian? A reflection on stochastic hydrogeology. Advances in Water Resources 21 (1), $47-61$.

Goovaerts, P., 1997. Geostatistics for natural resources evaluation. Oxford University Press, USA. 483pp.

Guardiano, F., Srivastava, R., 1993. Multivariate geostatistics: beyond bivariate moments. In: Soares, A. (Ed.), Geostatistics-Troia. Kluwer Academic Publ, Dordrecht, pp. 133-144.

Houtekamer, P. L., Mitchell, H. L., 2001. A sequential ensemble Kalman filter for atmospheric data assimilation. Monthly Weather Review 129 (1), 123137.

Journel, A. G., Deutsch, C. V., 1993. Entropy and spatial disorder. Mathematical Geology 25 (3), 329-355.

Kitanidis, P. K., 2007. On stochastic inverse modeling. In: Hyndman, D. W., Day-Lewis, F. D., Singha, K. (Eds.), Subsurface hydrology: Data integration for properties and processes. American Geophysical Union, Washington, DC, pp. 19-30. 


\section{A Normal-score transform}

Normal-score transform is a tool relating any distribution function $F(x)$ to a standard Gaussian function $G(y)$ (Goovaerts, 1997). It is described in Figure A.1, where the processes of normal-score transforming variable $x$ onto a Gaussian deviate $y$, and its inverse, are depicted. In other words, the two random variables $x$ and $y$ are linked through their cumulative probability distributions:

$$
\begin{array}{r}
F(x)=G(y) \\
y=G^{-1}[F(x)] \\
x=F^{-1}[G(y)]
\end{array}
$$

where $y$ follows a standard normal distribution with zero mean and unit variance and is the normal-score transform of $x$. More detailed information can be found in Goovaerts (1997) and Deutsch and Journel (1998).

For each one of the components of the state vector (hydraulic heads or hydraulic log-conductivities), the ensemble values are used to estimate a nonparametric cumulative distribution function $F(x)$. Estimation of $F(x)$ amounts to sort all values for a given state vector component, and assign to each value a cumulative probability equal to $i /\left(N_{r}+1\right)$, where $i$ is the ordinal position after sorting, and $N_{r}$ is the number of realizations in the ensemble. These distribution functions, which have to be computed for each location and each time step, are used to normal-score transform the components of the state vector, then the NS-EnKF is performed, and then a back transform is done to retrieve the state vector in its original space to feed it to the transfer function and perform the next forecast step. 


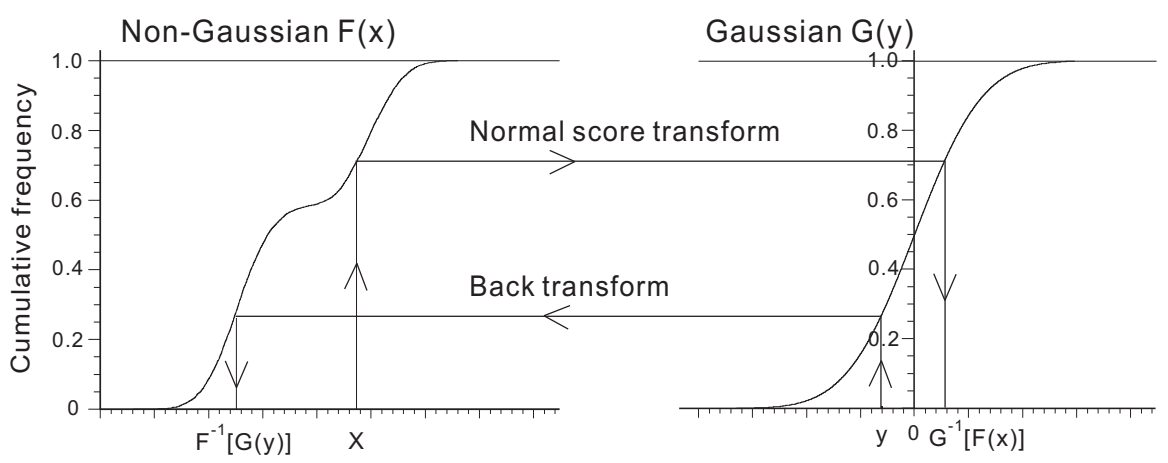

Figure A.1: Sketch of normal-score transformation.

In the back transform process, since $F(x)$ is non-parametrically defined, there is a need to establish rules of interpolation to retrieve the state vector value $x$ given a cumulative probability $F(x)$, which, most likely, will not correspond to any of the cumulative probabilities estimated from the values of the ensemble. Besides, since the Gaussian deviates could result in cumulative probabilities that are outside of the range of cumulative probabilities estimated from the ensemble, there is a need to explicitly state the absolute minimum and maximum of each state vector component, and the corresponding interpolation rules for the upper and lower tails of the cumulative distribution function. Different interpolation schemes are possible and the reader is referred to Deutsch and Journel (1998) for the details.

Considering the way the cumulative distribution function $F(x)$ is estimated, the backtransform $F^{-1} \cdot G(y)$ always exists; however, the normal-score transform $G^{-1} \cdot F(x)$ is undefined at conditioning locations for which all ensemble values are identical, and thus, $F(x)$ is a step function. At those locations, we have chosen to assign a normal score by taking the average of the normal scores of the state variables in the neighboring cells. 


\section{Bibliography}

Deutsch, C. V., Journel, A. G., 1998. GSLIB, Geostatistical Software Library and User's Guide, 2nd Edition. Oxford University Press, New York, 384pp.

Goovaerts, P., 1997. Geostatistics for natural resources evaluation. Oxford University Press, USA. 483pp. 
